\title{
Métodos de Decomposição para Problemas de Multifluxo em Redes
}

Célia Regina do Carmo Moraes

Orientador: Prof. Dr. Marcos Nereu Arenales

Dissertação apresentada ao Instituto de Ciências Matemáticas de São Carlos, da Universidade de São Paulo, como parte dos requisitos

para obtenção do título de Mestre em

"Ciência de Computação e Matemática Computacional".

Cutt. - 
À Deus.

Aos meus pais, Antonio e Rosa, a minha avó Carmela, aos meus irmãos Willans, Cristina e Morgania e ao meu marido, Carlos com carinho, pelo apoio e incentivo. 


\section{Agradecimentos}

Ao meu orientador e Professor Dr. Marcos Nereu Arenales pela orientação, dedicação, compreensão e paciência no desenvolvimento e conclusão deste trabalho.

Aos professores José Franscisco Ferreira, Antonio Castelo Filho e a professora Neide Maria B. Franco, com os quais tive a oportunidadede me aperfeiçoar através das disciplinas cursadas, e em especial a professora Neide B. Franco pela orientação acadêmica.

Aos meus professores Wilson Mauricio Tadini, Euripedes Alves da Silva e Antonio Espada Filho, com os quais tive a oportunidade de cursar disciplinas na graduação, pelo apoio e incentivo.

As secretárias Beth, Laura e Marília pela atenção e o bom atendimento que sempre recebi na seção de pós-graduação. Ao Jacques (xerox) e as bibliotecárias pela disponibilidade e compreensão.

Aos meus amigos Návia, Estela, Simone Domingues, Delazir, Tânia Costa, Jilson, Miguel, Max, Jorge e Ruy pelo carinho e amizade.

Aos meus velhos amigos do IBILCE, Verinha, Ana Vitti, Marcelo (Trolha), Gilberto (Pastel), Luis Carlos (Coxinha), Renato e Robison e aos novos amigos que conquistei Rosana (Beleza) e Moacir, Neusa e Ricardo, Mauro, Sadao, Maristela, Simone, Luciane Grossi e Marquinhos, Clébia, Lana, Esdras, Carlos, Cláudia (pura), Marcelo Maringá, Lucina Martinez, Rosângela, Lauro, Adriana, Elisangela, Cris Nogueira e a Marlene (mãezona) pela amizade e apoio nos momentos dificeis, mas muito mais pelas nossas risadas e o nosso convívio.

E a todas as outras pessoas que direta ou indiretamente ajudaram na elaboração deste trabalho.

Agradeço a CAPES pelo apoio financeiro. 


\section{Resumo}

Este trabalho apresenta um estudo sobre problemas de multifluxo em redes, juntamente com três abordagens de solução: o Particionamento Primal, a Decomposição RecursoDiretor e a Decomposição Dantzig-Wolfe.

O produto num problema de multifluxo pode ser definido de três maneiras. Cada uma dessas definições para produto produz uma formulação distinta para o problema de multifluxo: o Problema Produto Específico (PPE), o Problema do Destino Específico (PDE) e o Problema Origem e Destino Específico (POD). Neste trabalho foi feita uma investigação do impacto das três formulações sobre a decomposição de Dantzig-Wolfe. 


\section{Abstract}

This work presents a study on multicommodity network flows problem and three solution approaches: Primal Partitioning, Resourse-directive Decomposition, Dantzig-Wolfe decomposition.

A commodity in a network flow problem can be formulated by three ways leading to three different mathematical models: product specific problem (PSP), destination specific problem (DSP) and origin-destination problem (ODP). We also present an investigation of impact of problem formulation on Dantzig-Wolfe decomposition. 


\section{Índice}

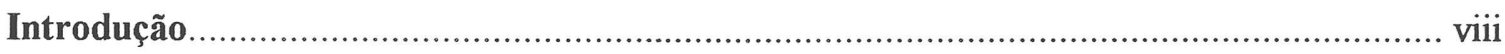

\section{Capítulo 1 Fluxo em redes}

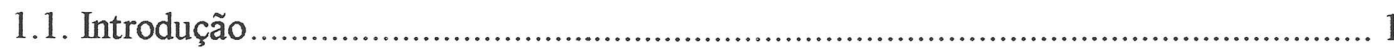

1.2. A definição do Problema de Fluxo em Rede de Custo Mínimo ................................. 1

1.3. O problema do Caminho Mínimo .......................................................................... 2

1.4. O Problema do Fluxo Máximo................................................................................. 4

1.5. Caracterização de base para rede .................................................................. 5

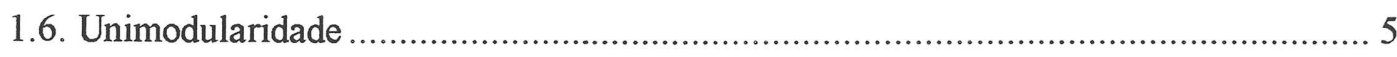

1.7. Especializações do Método Simplex para Redes ..................................................... 6

1.7.1 Cálculo dos potenciais dos nós (variáveis duais) …................................... 7

1.7.2 Algoritmo para determinação dos potenciais dos nós …............................. 7

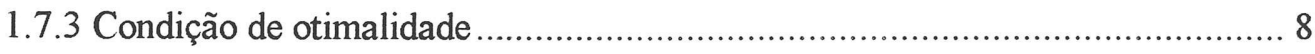

1.7.4 Determinação do arco a entrar na base ................................................... 9

1.7.5 Determinação da direção de perturbação das variáveis ................................ 9

1.7.6 Determinação de $\Delta$ (o valor da alteração dos fluxos) ................................. 11

1.7.7 Algoritmo simplex sobre uma rede ..................................................... 13

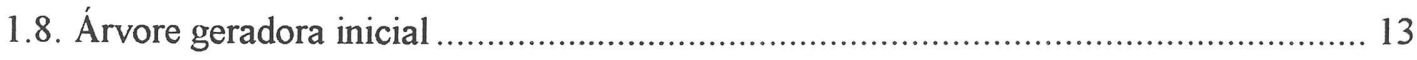

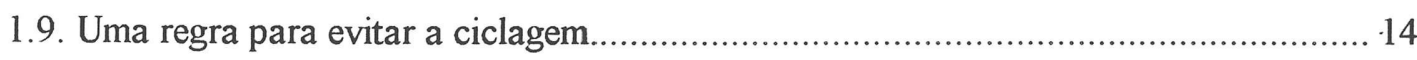

\section{Capítulo 2 Multifluxo em Redes}

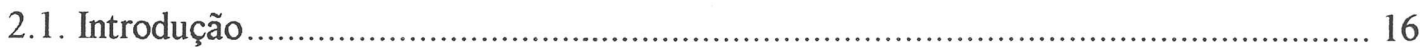

2.2. Definição đ́o Problema de Multifluxo em Redes ...................................................... 16

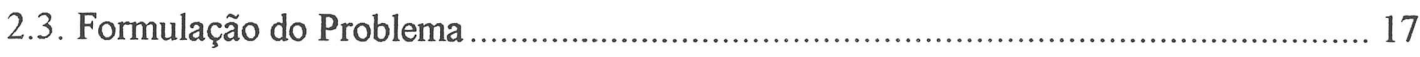

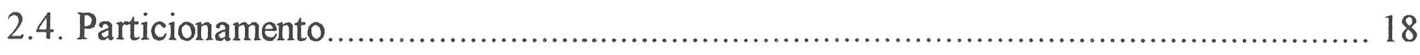

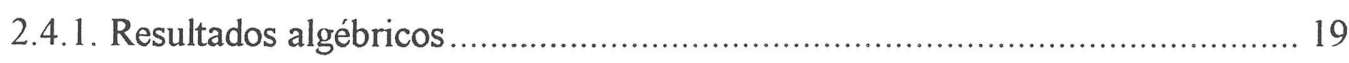




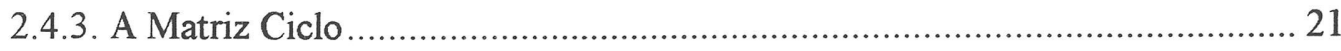

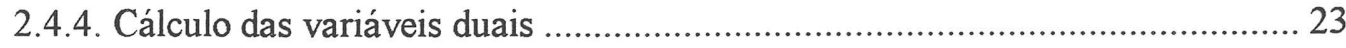

2.4.5. Cálculo da direção de perturbação para as variáveis básicas .......................... 24

2.4.6. Algoritmo simplex especializado para o Problema de Multifluxo.................... 25

2.4.7. Atualização da inversa da Matriz Ciclo ....................................................... 26

\section{Capítulo 3 A Decomposição para Problemas de Multifluxo em Redes}

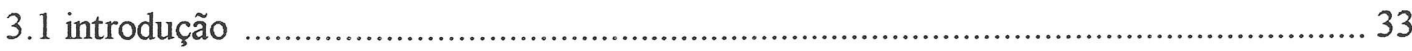

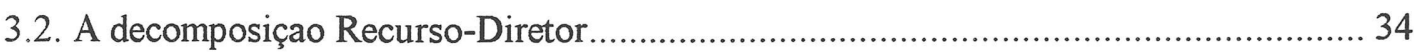

3.2.1. Resolvendo o problema mestre - Otimização do Subgradiente ........................ 35

3.2.2 Otimização do Subgradiente para problemas de multifluxo ............................. 39

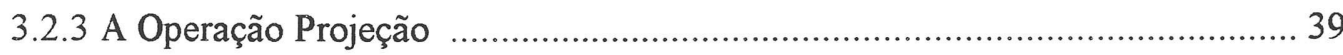

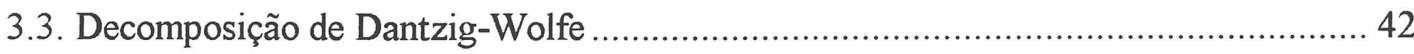

3.3.1. Decomposição de Dantzig-Wolfe para Problema de Multifluxo em Redes ...... 42

3.3.2 Algoritmo de Decomposição de Dantzig-Wolfe .......................................... 51

\section{Capítulo 40 Impacto da Formulação sobre a Decomposição de Dantzig-Wolfe}

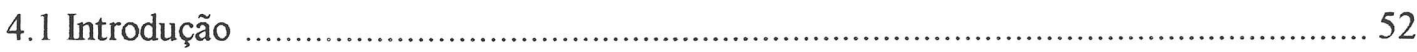

4.2 As Três Formulações para Problemas de Multifluxo ................................................ 53

4.3 A decomposição Dantzig-Wolfe para as Três Formulações ....................................... 57

4.3.1 A Formulação do Problema Mestre Restrito..................................................... 57

4.3.2 A Formulação do Subproblemas .............................................................. 58

4.4 Comparação das Formulações ……................................................................... 59

4.5 Uma Formulação Baseada em Caminhos para PPE ................................................... 62

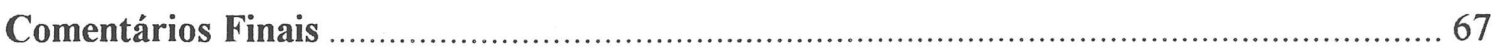

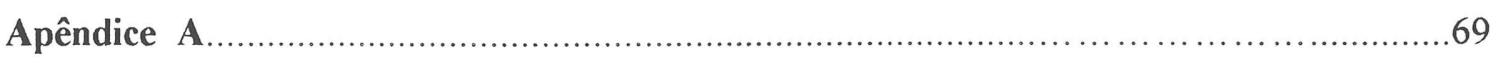

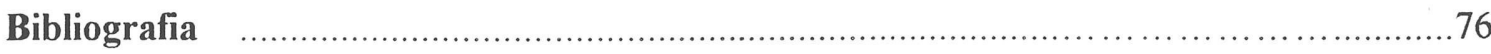




\section{Introdução}

Problemas de fluxo em redes têm sido objeto de pesquisas nas mais diversas áreas. Suas idéias básicas surgiram com Gustav Kirchhoff, entre outros pioneiros da Engenharia Elétrica, que analisando pela primeira vez os circuitos elétricos sistematicamente, fundamentaram muitas das idéias chaves da teoria de fluxo em redes e estabeleceram redes (grafos) como objetos matemáticos usuais para a representação de sistemas físicos.

Com o desenvolvimento do algoritmo simplex por George Dantzig, os modelos de fluxo em redes foram extensivamente estudados. $\mathbf{O}$ grande interesse nesta classe de problemas foi motivado pelas numerosas aplicações. Os modelos de fluxo em redes exibem uma estrutura especial, que pode ser explorada para o desenvolvimento de técnicas especializadas.

Em muitas aplicações, diversos produtos compartilham a mesma rede. Se esses produtos não interagem de nenhuma maneira, para resolver esse problema basta que resolvamos um conjunto de problemas de fluxo em rede, um para cada produto. Entretanto, se este não é o caso, para encontrar o fluxo ótimo, os problemas devem ser resolvidos de comun acordo com os demais. Este problema é conhecido como problema de Multifluxo .

Os problemas de multifluxo apresentam uma estrutura especial, bloco-angular com restrições adicionais, o que possibilita o desenvolvimento de técnicas especializada para resolvê-los.

O objetivo deste trabalho é estudar problemas de multifluxo em redes, com uma visão geral das abordagens básicas de soluções baseadas em decomposição: o Particionamento, a Decomposição Recurso-Diretor e a Decomposiçãode Dantzig-Wolfe.

Este trabalho está organizado da seguinte forma:

No capítulo 1 , apresentamos os problemas de fluxo em rede de custo mínimo e o do caminho mínimo bem como as suas variações. Tais problemas são considerados problemas de fluxo em rede "puros" e serão importantes no desenvolvimento deste trabalho.

No capítulo 2 apresentamos o problema de multifluxo em redes, juntamente o Particionamento Primal. 
No capítulo 3 as outras duas abordagens de decomposição são apresentadas: a Decomposição Recurso-Diretor e a Decomposição de Dantzig-Wolfe.

No capítulo 4 são apresentadas três formulações para o problema de multifluxo e um estudo do impacto das formulações sobre a Decomposição de Dantzig-Wolfe. 


\section{Capítulo 1}

\section{Fluxo em Redes}

\subsection{Introdução}

Os modelos matemáticos de fluxo em redes que serão apresentados neste capítulo, para os problemas tais como de fluxo em rede de custo mínimo, do caminho mínimo, do fluxo máximo são chamados problemas de fluxo em rede puros. Tais modelos podem estar imersos em várias aplicações que possuam uma estrutura de rede, mas também variáveis e/ou restrições adicionais. Em particular, os problemas de fluxo em rede de custo mínimo e do caminho mínimo serão de especial importância para o estudo de multifluxos.

Neste capítulo apresentaremos também uma especialização do método simplex para problemas de fluxo em redes. No apêndice A encontram-se as definições e proposições básicas da teoria dos grafos que serão utilizadas durante o decorrer deste trabalho.

\subsection{A Definição do Problema de fluxo em rede de custo mínimo}

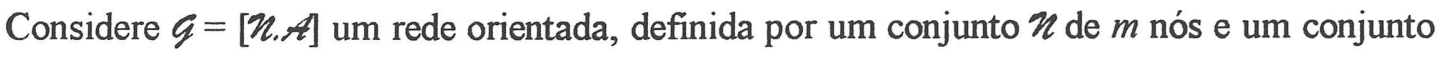
$A$ de $n$ arcos. Associadas a esta rede existem algumas quantidades que possibilitam a definição e a apresentação do modelo matemático de um problema de fluxo em rede de custo mínimo.

Cada arco $e_{\mathrm{j}}=(\mathrm{F}(\mathrm{j}), \mathrm{T}(\mathrm{j})) \in \mathscr{A}$ tem um custo associado $\mathrm{c}_{\mathrm{j}}$, que denota o custo por unidade de fluxo sobre este arco. Será assumido que o custo do fluxo sobre cada arco $e_{\mathrm{j}}$ varia linearmente com a quantidade de fluxo sobre este arco. Associado também a cada arco $e_{\mathrm{j}}$ existe um limitante para a quantidade máxima de fluxo que pode passar através deste arco, chamada de capacidade do arco e denotada por $\mathrm{u}_{\mathrm{j}}$.

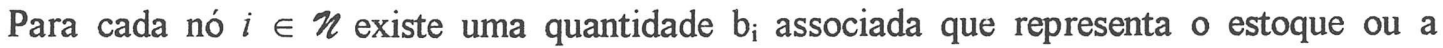
demanda do nó. Se $b_{\mathrm{i}}>0$, o nó $i$ é um nó estoque, com estoque igual a $b_{\mathrm{i}}$; se $\mathrm{b}_{\mathrm{i}}<0$, então o nó $i$ é um nó demanda, com demanda igual a $\left|\mathrm{b}_{\mathrm{i}}\right| ; \mathrm{se}_{\mathrm{i}}=0$, o nó $i$ é um ponto de passagem.

Definidas e determinadas estas quantidades, o problema de fluxo em rede de custo mínimo consiste em determinar o fluxo de um único produto através de uma rede dada, satisfazendo as 
demandas de determinados nós a partir de estoques disponíveis em outros nós, de maneira que o custo total deste produto que percorre a rede seja mínimo.

As variáveis de decisão para o problema de fluxo em rede são os fluxos sobre os arcos e será representado por $\mathrm{x}_{\mathbf{j}}$. como:

O problema de fluxo em rede de custo mínimo pode ser apresentado na forma matricial

$$
\begin{array}{ll}
\text { Minimizar } & \mathbf{c x} \\
\text { sujeito a } & \mathbf{A x}=\mathbf{b} \\
& \mathbf{0} \leq \mathbf{x} \leq \mathbf{u}
\end{array}
$$

Nesta formulação, A é a matriz incidência nó-arco. As restrições (1.1b) são chamadas de restrições conservação de fluxo ou restrições de equilíbrio de massa. Estas restrições estabelecem que a quantidade total de fluxo que sai do nó menos a quantidade total de fluxo que entra, deve ser igual ao estoque ou a demanda deste nó. As restrições (1.1c) são as restrições de capacidade de fluxo sobre cada arco. Existem problemas para os quais o fluxo sobre um, ou mais arcos não são limitados ( isto é, $\mathrm{u}_{\mathrm{j}}=\infty$ ), ou até mesmo para todo arco $e_{\mathrm{j}}$ na rede. Quando isto acontece, tem-se somente as restrições de não-negatividade $(\mathbf{x} \geq \mathbf{0})$.

\subsection{O Problema do Caminho Mínimo}

Outro problema de fluxo em rede importante e que pode ser apresentado como caso especial do problema de fluxo em rede de custo mínimo é o problema do caminho mínimo, que aparece freqüentemente em uma variedade de aplicações.

Dada uma rede com $m$ nós e $n$ arcos, o problema do caminho mínimo consiste em encontrar o caminho de comprimento (ou custo) mínimo, a partir de um nó estoque $s$ específico, para um outro nó demanda $t$ específico, onde $(\mathrm{s}, \mathrm{t})$ pode ser qualquer par de nós dado e cada arco $e_{\mathrm{j}} \in \mathscr{A}$ tem comprimento (ou custo) associado $c_{j}$.

Fazendo $b_{s}=1, b_{t}=-1$ e $b_{i}=0$ para todos os outros nós na rede do problema de fluxo de custo mínimo, o problema do caminho mínimo pode ser apresentado na forma matricial como:

$$
\begin{gathered}
\text { Minimizar } \mathbf{c x} \\
\text { sujeito a } \quad \mathbf{A x}=\mathrm{b} \\
\text { onde } \mathrm{b}_{\mathrm{i}}= \begin{cases}1 & \text { se } \mathrm{i}=\mathrm{s} \\
0 & \text { se } \mathrm{i} \neq \mathrm{s} \text { ou } \mathrm{t} \quad \mathrm{i}=1, \ldots, m \\
-1 & \text { se } \mathrm{i}=\mathrm{t} \text {. }\end{cases}
\end{gathered}
$$

A restrição $\mathrm{x}_{\mathrm{j}}=0 / 1$ indica se o arco $e_{\mathrm{j}}$ está ou não no caminho.

$\mathrm{Na}$ seção 1.6 veremos que a matriz incidência nó-arco, associada as restrições de conservação de fluxo de uma rede, é totalmente unimodular. Se o vetor de estoques/demandas b é 
inteiro, o problema acima sempre tem solução ótima inteira. Portanto, no problema inteiro (1.2) a restrição $x_{j}=0 / 1$ pode ser substituída pela restrição $x_{j} \geq 0$, tornando-se um programa linear.

$$
\begin{gathered}
\text { Minimizar cx } \\
\text { sujeito a } \quad \mathbf{A x}=\mathrm{b} \\
\text { onde } \mathrm{b}_{\mathrm{i}}= \begin{cases}1 & \text { se } \mathrm{i}=\mathrm{s} \\
0 & \text { se } \mathrm{i} \neq \mathrm{s} \text { ou } \mathrm{t} \quad \forall \quad \mathrm{i}=1, \ldots, m \\
-1 & \text { se } \mathrm{i}=\mathrm{t} .\end{cases}
\end{gathered}
$$

A solução deste problema enviará um unidade de fluxo a partir do nó $s$ para o nó $t$ ao longo de um caminho mínimo.

Esta formulação para o problema do caminho mínimo também modela situações nas quais pretende-se enviar uma certa quantidade de fluxo $f$, a partir de um nó estoque $s$ específico, para um outro nó demanda $t$ específico ao longo de um caminho mínimo, onde agora tem-se $\mathrm{b}_{\mathrm{s}}=f$ e $\mathrm{b}_{\mathrm{t}}=-f$.

A solução para este problema enviará $f$ unidades de fluxo a partir do nó estoque $s$ para o nó demanda $t$, ao longo de um caminho mínimo desde que os arcos da rede não sejam capacitados, ou tenham capacidade no mínimo $f$.

$$
\begin{aligned}
& \text { Minimizar cx } \\
& \text { sujeito a } \mathbf{A x}=\mathbf{b} \\
& \text { onde } b_{i}= \begin{cases}f & \text { se } i=s \\
0 & \text { se } i \neq s \text { ou } t \quad i=1, \ldots, m \\
-f & \text { se } i=t .\end{cases}
\end{aligned}
$$

Este tipo de problema do caminho mínimo, aparece como subproblema para problemas de multifluxo em redes, quando estes estão formulados de uma maneira específica que será apresentada no capítulo 3.

Alternativamente, pode-se ver o problema do caminho mínimo como o de encontrar o caminho de comprimento (ou custo) mínimo a partir de um nó estoque $s$ específico para todo outro nó em $\eta-\{s\}$. Este ponto de vista fornece a seguinte formulação para o problema do caminho mínimo:

$$
\begin{aligned}
& \text { Minimizar cx } \\
& \text { sujeito a } \mathbf{A x}=\mathbf{b} \\
& \mathrm{x}_{\mathrm{j}} \geq 0 \quad \forall e_{\mathrm{j}} \in \mathscr{A} \\
& \text { onde } b_{i}=\left\{\begin{array}{lc}
m-1 & \text { se } i=s \\
-1 & \forall i \in n-\{s\}
\end{array} \quad \mathrm{i}=1, \ldots, m .\right.
\end{aligned}
$$

A solução para esta formulação do problema do caminho mínimo é chamada árvore do caminho mínimo, pois a solução é uma árvore formada por caminhos mínimos a partir do estoque $s$ específico para todos os outros nós destino $t \in \mathbb{N}-\{s\}$. 
Da mesma maneira que a anterior, esta formulação também modela situações nas quais pretende-se enviar uma certa quantidade de fluxo a partir de um nó estoque $s$ específico para todos os outros nós em $\mathscr{U}-\{s\}$ da rede. Entretanto, a demanda agora pode variar para cada nó de $\mathscr{U}-\{s\}$. Logo, o nó estoque $s$ específico deverá ter estoque igual a soma de todas as demandas.

$$
\begin{aligned}
& \text { Minimizar cx (1.6a) } \\
& \text { sujeito } \mathbf{a} \quad \mathbf{A x}=\mathbf{b} \\
& \mathrm{x}_{\mathrm{j}} \geq 0 \quad \forall e_{\mathrm{j}} \in \mathscr{A} \\
& \text { onde } \mathrm{b}_{\mathrm{i}}=\left\{\begin{array}{ll}
\sum f_{\mathrm{i}} & \text { se } \mathrm{i}=\mathrm{s} \\
-f_{\mathrm{i}} & \forall \mathrm{i} \in \boldsymbol{U}-\{\mathrm{s}\}
\end{array} \quad \mathrm{i}=1, \ldots, m .\right.
\end{aligned}
$$

Problemas do caminho mínimo com esta formulação também aparecem como subproblemas em problemas de multifluxo em redes devido a uma outra formulação específica, que também será apresentada no capítulo 3 .

\subsection{O Problema do fluxo máximo}

O problema de fluxo máximo é um modelo complementar do problema do caminho mínimo, no sentido de que, o problema do caminho mínimo modela situações nas quais o fluxo está sujeito a um custo, mas não está restrito pela capacidade.

O problema do fluxo máximo procura uma solução factível que envia uma quantidade máxima de fluxo a partir de um nó estoque $s$ específico para um outro nó demanda $t$ específico, sem que as restrições de capacidade $\mathbf{0} \leq \mathbf{x} \leq \mathbf{u}$ sejam violadas.

Seja $f$ é o fluxo na rede a partir do nó $s$ para o nó $t$ que pretende-se maximizar. Fazendo $b_{\mathrm{i}}=0$ para todo nó $i$ e $c_{\mathrm{j}}=0$ para todo arco $e_{\mathrm{j}}$ na rede do problema de fluxo de custo mínimo, o problema do fluxo máximo pode ser apresentado na forma matricial como:

$$
\begin{array}{ll}
\text { Maximizar } f & \\
\text { sujeito a } & \mathbf{A x}+f\left(\mathrm{e}^{\mathrm{t}}-\mathbf{e}^{\mathrm{s}}\right)=\mathbf{0} \\
& 0 \leq \mathrm{x}_{\mathrm{j}} \leq \mathrm{u}_{\mathrm{j}} \quad \forall e_{\mathrm{j}} \in \mathscr{A}
\end{array}
$$

onde A é a matriz incidência nó-arco.

Note que no segundo termo nas restrições de conservação de fluxo, tem-se a diferença entre dois vetores unitários associados ao fluxo $f$. Portanto, $f$ pode ser visto como a variável fluxo a partir do nó $t$ para o nó $s$, ou seja $f \equiv \mathrm{x}_{\mathrm{n}+1}$, onde $e_{\mathrm{n}+1}=(\mathrm{t}, \mathrm{s})$. Isto corresponde a acrescentar o arco $e_{\mathrm{n}+1}=(\mathrm{t}, \mathrm{s})$ na rede. Portanto, o problema de fluxo máximo pode ser visto como um caso particular do problema de fluxo em rede de custo mínimo dado por:

$$
\begin{array}{ll}
\text { Minimizar }-\mathrm{x}_{\mathbf{n}+1} & \\
\text { sujeito a } \quad \mathbf{A x} & =\mathbf{0} \\
& 0 \leq \mathrm{x}_{\mathrm{j}} \leq \mathrm{u}_{\mathrm{j}} \quad \forall e_{\mathrm{j}} \in \mathcal{A} .
\end{array}
$$

onde A é a matriz incidência nó-arco para a rede, quando esta já está acrescentada do arco $e_{\mathrm{n}+1}=(\mathrm{t}, \mathrm{s}), \mathrm{u}_{\mathrm{n}+1}=\infty$ e $\mathrm{c}_{\mathrm{n}+1}=-1$. 


\subsection{Caracterização de base para rede}

Nesta seção estabelecemos a correspondência entre bases e árvores geradoras.

Pela proposição A.10 (apêndice A), tem-se que a matriz de restrições para o problema de fluxo em rede de custo mínimo (PR), tem posto um a menos que o número de linhas (nós).

Pode-se reformular o problema de maneira que a matriz incidência nó-arco tenha posto linha completo, adicionando uma coluna linearmente independente (variável), tendo limite superior igual a zero, resultando na seguinte formulação para o problema de fluxo em rede de custo mínimo:

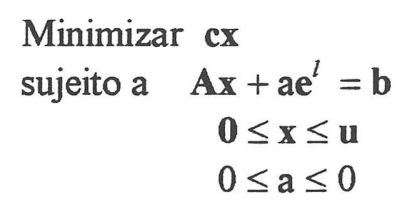

onde $\mathrm{e}^{l}$ é um vetor com $m$ componentes, cuja $l$-ésima tem valor igual a "1" e as demais iguais a zero.

O grafo associado com o problema (1.9) é chamado grafo enraizado, a é chamado arco raiz e o nó $l$ como nó raiz. A árvore associada a este grafo é chamada árvore enraizada e o arco raiz é representado por um arco incidente sobre um único nó (raiz).

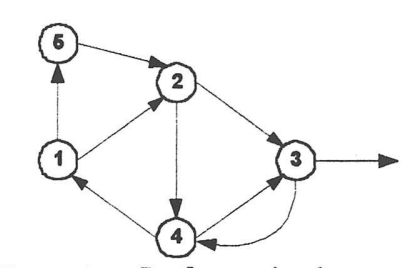

Figura 1.a: Grafo enraizado

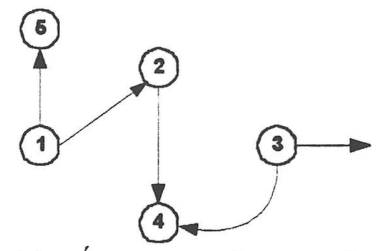

Figura 1.b: Árvore geradora enraizada

Proposição 1.1 Seja A a matriz incidência nó-arco para o grafo próprio conexo $\mathscr{q}=[\varkappa, \mathscr{A}]$ tendo $m$ nós. Seja $7=[\varkappa, \hat{\mathscr{A}}]$ uma árvore geradora para $\mathcal{q}$. Então $\Omega=\left\{\mathrm{A}^{\mathrm{j}} ; e_{\mathrm{j}} \in \hat{\mathscr{A}}\right\} \cup\left\{\mathrm{e}^{l}\right\}$ gera $\mathrm{R}^{\mathrm{m}}$.

Proposição 1.2 Seja $\mathbf{A}$ a matriz incidência nó-arco para o grafo próprio enraizado com nó raiz $l$. Toda base para $\left[\mathbf{A}, \mathbf{e}^{l}\right]$ deve ser formada por $\mathrm{e}^{l}$ juntamente com um conjunto de colunas de A que corresponde a uma árvore geradora para $\mathfrak{g}$.

Proposição 1.3 Seja A a matriz incidência nó-arco para o grafo próprio enraizado com nó raiz $l$. Seja $\mathbf{B}$ qualquer base para $\left[\mathbf{A}, \mathbf{e}^{l}\right]$. Então $\mathbf{B}$ é triangular inferior.

Esta propriedade é um fator importante para as especializações do algoritmo simplex para redes, que será apresentado posteriormente.

\subsection{Unimodularidade}

Nesta seção mostraremos que a matriz de restrições para o problema (1.9) é totalmente unimodular. Esta propriedade garante que toda solução básica factível para o problema é uma solução inteira, desde que o vetor de estoques/demandas (b) e o vetor de capacidades (u) sejam inteiros. 
Seja A uma matriz com elementos inteiros. Diz-se que uma matriz A é unimodular, se o determinante de toda matriz básica $\mathbf{B}$ para a matriz $\mathbf{A}$ é igual a \pm 1 .

A proposição a seguir mostra a relação entre unimodularidade e a solução inteira de programas lineares.

Proposição 1.4 (Unimodularidade) Seja A uma matriz inteira. Então as seguintes condições são equivalentes:

(1) A é unimodular;

(2) Cada solução básica factível definida pela restrições $\mathbf{A x}=\mathbf{b}$ e $\mathbf{0} \leq \mathbf{x} \leq \mathbf{u}$, é inteira para quaisquer vetores inteiros $\mathbf{b}$ e $\mathbf{u}$;

(3) Cada matriz básica $\mathbf{B}$ de $\mathbf{A}$ tem uma inversa inteira $\mathbf{B}^{-1}$.

Para estabelecer uma conexão entre fluxos em redes e o resultado da proposição anterior, deve-se considerar uma outra classe de matrizes: as totalmente unimodulares.

Diz-se que uma matriz A é totalmente unimodular, se cada submatriz quadrada de $\mathbf{A}$ tem determinante igual a 0 ou \pm 1 .

Proposição 1.5 A matriz incidência nó-arco de uma rede orientada é totalmente unimodular.

Toda matriz totalmente unimodular $\mathbf{A}$, é uma matriz unimodular, pois cada matriz básica $\mathbf{B}$ para $\mathbf{A}$ deve ter determinante \pm 1 . Portanto, como a matriz incidência nó-arco para a rede é totalmente unimodular e os dados do problema (capacidades, estoques/demandas e os custos) são inteiros, toda solução básica factível é uma solução inteira.

Proposição 1.6 (Integralidade primal) Se a capacidade de todos os arcos e o estoque/demanda de todos os nós são inteiros, o problema de fluxo de custo mínimo sempre tem uma solução ótima inteira.

Proposição 1.7 (Integralidade Dual) Se todos os custos são inteiros, o problema de custo mínimo tem variáveis duais ótimas inteiras.

\subsection{Especializações do método Simplex para redes}

Como vimos pela proposição 1.2 , uma base para matriz incidência nó-arco $\left[\mathbf{A}, \mathbf{e}^{\mathbf{l}}\right]$ do problema (1.9) é uma árvore geradora juntamente com o arco raiz. Portanto com relação a partição básica para o método simplex, nos referimos a $[\mathbf{B}, \mathbf{N}]$ como estrutura árvore geradora e a árvore geradora enraizada associada a base $\mathbf{B}$ como árvore básica, denotada por $7_{\mathrm{B}}$. Os $\operatorname{arcos} e_{\mathrm{j}} \in 7_{\mathrm{B}}$ são chamados arcos básicos e os que não pertencem como arcos não-básicos. O fluxo sobre os arcos não-básicos são dados por : $x_{j}=0$ ou $x_{j}=u_{j}$. 


\subsubsection{Cálculo dos potenciais dos nós (variáveis duais)}

Dado que a base para o problema (1.9) é triangular (proposição 1.3) podemos simplificar algumas operações do método simplex envolvendo a base $\mathbf{B}$ e sua inversa $\mathbf{B}^{-1}$. Uma dessas operações envolvendo a inversa da matriz básica é utilizada para determinar os valores das variáveis duais $\pi$ 's, dada por $\pi=\mathbf{c}^{\mathbf{B}} \mathbf{B}^{-1}$, que em problemas de fluxo em redes são chamadas de potenciais dos nós.

Mas $\pi=\mathbf{c}^{\mathbf{B}} \mathbf{B}^{-1}$ é a solução do sistema de equações lineares $\pi \mathbf{B}=\mathbf{c}^{\mathbf{B}}$. Como $\mathbf{B}$ é triangular, $\pi$ pode ser obtido diretamente por substituições recursivas.

Em geral, para uma base $\mathbf{B}$ com nó raiz $l, \pi \mathbf{B}=\mathbf{c}^{\mathbf{B}}$ se reduz em:

$$
\begin{aligned}
& \pi_{l}=0 \\
& \pi_{\mathrm{F}(\mathrm{j})}-\pi_{\mathrm{T}(\mathrm{j})}=\mathrm{c}_{\mathrm{j}} \quad \forall e_{\mathrm{j}}=(\mathrm{F}(\mathrm{j}), \mathrm{T}(\mathrm{j})) \in \mathbf{7}_{\mathrm{B}} .
\end{aligned}
$$

Uma outra maneira de determinar os potenciais dos nós $(\pi)$ através da árvore básica sem que seja necessário a resolução de um sistema é dado pelo algoritmo a seguir.

\subsubsection{Algoritmo para Determinação dos potenciais dos nós (variáveis duais)}

0. Inicialização:

Seja $7=[n, \nexists]$ a árvore geradora básica com nó raiz $l$

$\pi_{l}=0, \quad n^{\mathrm{L}}=\{l\}$ e $n^{\mathrm{U}}=n-\{l\}$.

$\eta^{\mathrm{L}}$ : conjunto de nós rotulados.

1. Encontrar um arco $e_{j}$ cujo nó $T(j)$ pertença ao conjunto dos nós rotulados:

Seja $e_{\mathrm{j}} \in \mathscr{A}$ tal que $\mathrm{F}(\mathrm{j}) \in \varkappa^{\mathrm{U}}$ e $\mathrm{T}(\mathrm{j}) \in \varkappa^{\mathrm{L}}$.

Se tal arco não existir, vá para o passo 3 .

2. Rotulação do nó $F(j)$ :

Faça $\pi_{\mathrm{F}(\mathrm{j})}=\mathrm{c}_{\mathrm{j}}+\pi_{\mathrm{T}(\mathrm{j})}$

Retorne ao passo 1 .

$$
x^{\mathrm{L}}=x^{\mathrm{L}} \cup\{\mathrm{F}(\mathrm{j})\} \text { e } x^{\mathrm{U}}=x^{\mathrm{U}}-\{\mathrm{F}(\mathrm{j})\} .
$$

3. Encontrar um arco $e_{\mathrm{j}}$ cujo o nó $F(j)$ pertença ao conjunto dos nós rotulados:

Seja $e_{\mathrm{j}} \in \mathscr{A}$ tal que $\mathrm{T}(\mathrm{j}) \in \boldsymbol{\varkappa}^{\mathrm{U}}$ e $\mathrm{F}(\mathrm{j}) \in \boldsymbol{H}^{\mathrm{L}}$.

Se tal arco não existe, termine.

4. Rotulação do nó $T(j)$ :

Faça $\pi_{\mathrm{T}(\mathrm{j})}=-\mathrm{c}_{\mathrm{j}}+\pi_{\mathrm{F}(\mathrm{j})}$

$$
x^{\mathrm{L}}=x^{\mathrm{L}} \cup\{\mathrm{T}(\mathrm{j})\} \text { e } \boldsymbol{x}^{\mathrm{U}}=\boldsymbol{x}^{\mathrm{U}}-\{\mathrm{T}(\mathrm{j})\} .
$$

Retorne ao passo 1 .

Através do algoritmo 1.7.2, todos os potenciais dos nós (variáveis duais) são unicamente determinados, pois a árvore básica é conexa e não tem ciclos. 


\subsubsection{Condição de Otimalidade}

Um dos aspectos importantes das condições de otimalidade para o método simplex, é que elas fornecem um mecanismo simples e eficaz de verificar se uma solução dada, determina de fato, em se tratando de um problema de minimização, o mínimo para este problema.

No método simplex uma partição é feita na matriz de restrições $\mathbf{A}=[\mathbf{B}, \mathbf{N}]$, determinando assim uma matriz básica B e uma outra matriz não-básica $\mathbf{N}$. Associada a esta partição existe uma solução $\mathbf{x}=\left[\mathrm{x}^{\mathrm{B}}, \mathbf{x}^{\mathrm{N}}\right]$. Para verificar se essa é a melhor solução para o problema, isto é, a ótima, devese analisar se a partição feita é a ótima, ou seja, verificar se nenhuma das colunas associadas a matriz não-básica $\mathbf{N}$, não deveriam estar compondo a matriz básica $\mathbf{B}$.

Um conceito chave para a apresentação das condições de otimalidade é a do chamado custo reduzido, denotado por $\mathrm{c}_{\mathrm{j}}^{\pi}$.

$\mathrm{O}$ custo reduzido é um custo atribuído aos arcos não-básicos dado por:

$$
\begin{aligned}
& c_{j}^{\pi}=c_{j}-\pi N^{j} \text { se } x_{j}=0 \text { e } \\
& c_{j}^{\pi}=\pi N^{j}-c_{j} \text { se } x_{j}=u_{j}
\end{aligned}
$$

onde $\mathbf{N}^{\mathrm{j}}$ é a coluna não-básica correspondente ao arco não-básico $e_{\mathrm{j}}$ e $\mathrm{c}_{\mathrm{j}}$ é o custo deste arco. Como cada coluna não-básica $\mathbf{N}^{j}$ tem somente dois elementos não-nulos (+1 na componente correspondente ao nó $F(j)$ e -1 na componente correspondente ao nó $T(j))$, o custo reduzido pode ser dado por:

$$
\begin{aligned}
& c_{j}^{\pi}=c_{j}-\pi_{F(j)}+\pi_{T(j)} \text { se } \quad x_{j}=0 \text { e } \\
& c_{j}^{\pi}=\pi_{F(j)}-\pi_{T(j)}-c_{j} \text { se } \quad x_{j}=u_{j} .
\end{aligned}
$$

Portanto o custo reduzido é determinado pelo custo original do arco não-básico mais a variação dos potenciais dos nós incidentes neste arco.

Pelo apêndice B pode-se observar que, se o custo relativo é negativo, isto é, $c_{j}^{\pi}<0$ para algum arco não-básico $e_{\mathrm{j}}$, o valor da função objetivo para o problema ainda pode ser menor que o valor que a solução corrente oferece.

A proposição a seguir estabelece as condições para que a árvore básica seja ótima para o problema de fluxo em rede de custo mínimo.

Proposição 1.8 Seja 7B uma árvore básica factível para o problema de fluxo em rede de custo mínimo. Se para algum valor dos potenciais dos nós $(\pi)$, o custo reduzido $c_{j}^{\pi}$ satisfaz as seguintes condições:

(i) $\mathrm{c}_{\mathrm{j}}^{\pi} \geq 0$ para todo arco $e_{\mathrm{j}} \notin 7_{\mathrm{B}}$ tal que $\mathrm{x}_{\mathrm{j}}=0$; e

(ii) $\mathrm{c}_{\mathrm{j}}^{\pi} \leq 0$ para todo arco $e_{\mathrm{j}} \notin 7_{\mathrm{B}}$ tal que $\mathrm{x}_{\mathrm{j}}=\mathrm{u}_{\mathrm{j}}$,

então a árvore básica $7_{\mathrm{B}}$ é ótima.

A condição suficiente de otimalidade apresentada na proposição anterior é também necessária se a hipótese de não-degeneração da solução básica é assumida. 


\subsubsection{Determinação do arco a entrar na base}

Se a árvore básica satisfaz a proposição 1.8 , o algoritmo simplex termina, com a solução corrente ótima. Caso contrário o algoritmo seleciona um arco $e_{\mathrm{k}}=(\mathrm{F}(\mathrm{k}), \mathrm{T}(\mathrm{k}))$ violando as condições de otimalidade. Assim, os conjuntos dos arcos não-básicos candidatos a alterar o fluxo na rede, ou seja, serem inseridos na árvore básica $7_{\mathrm{B}}$ são dados por:

$$
\begin{aligned}
& \Psi_{1}=\left\{e_{\mathrm{k}} ; \mathrm{x}_{\mathrm{k}}=0 \text { e } \mathrm{c}_{\mathrm{k}}-\pi_{\mathrm{F}(\mathrm{k})}+\pi_{\mathrm{T}(\mathrm{k})}<0\right\} \\
& \Psi_{2}=\left\{e_{\mathrm{k}} ; \mathrm{x}_{\mathrm{k}}=u_{\mathrm{k}} \text { e } c_{\mathrm{k}}-\pi_{\mathrm{F}(\mathrm{k})}+\pi_{\mathrm{T}(\mathrm{k})}>0\right\}
\end{aligned}
$$

Portanto, o algoritmo seleciona um arco $e_{\mathrm{k}} \in \psi_{1} \cup \psi_{2}$ para entrar na árvore básica.

Pode existir mais que um arco no conjunto $\psi_{1} \cup \psi_{2}$ candidato a alterar o fluxo na rede. Entretanto, apenas um arco será selecionado.

Existem regras eficientes para selecionar tal arco, como por exemplo:

- Regra de seleção de Dantzig: onde a cada iteração esta regra seleciona uma arco para entrar árvore básica com violação máxima da condição de otimalidade $\left(\left|c_{j}^{\pi}\right|\right)$. A motivação para a utilização desta regra é que selecionar um arco dessa forma implica em um decréscimo maior na função objetivo.

- Regra de seleção do Primeiro arco ilegível: onde o arco selecionado para entrar na árvore básica é o primeiro arco encontrado que não satisfaz a condição de otimalidade. Na iteração seguinte, a procura na lista começa a partir do último arco selecionado. Se a procura for até o final desta lista, volta-se a procurar desde o início da lista. A motivação para utilizar esta regra é que ela identifica rapidamente o arco entrando.

\subsubsection{Determinação da direção de perturbação das variáveis}

Depois de selecionado o arco não-básico $e_{\mathrm{k}}$, adicionando este arco na árvore básica um ciclo é formado. $\mathrm{O}$ fluxo sobre $e_{\mathrm{k}}$ será alterado por $\Delta$ unidades, portanto, os fluxos sobre todos os arcos básicos ao longo do ciclo formado também devem ser alterados por $\Delta$ unidades, para que as restrições de conservação de fluxo sejam satisfeitas.

Seja $e_{\mathrm{k}}=(\mathrm{F}(\mathrm{k}), \mathrm{T}(\mathrm{k}))$ o arco não-básico selecionado. A alteração do fluxo sobre o arco nãobásico é dada a seguir.

Se o arco selecionado $e_{\mathrm{k}}$ tem fluxo $\mathrm{x}_{\mathrm{k}}=0$, ou seja $e_{\mathrm{k}} \in \psi_{1}$, deve-se aumentar o fluxo sobre este arco, então $\mathrm{x}_{\mathrm{k}}=\mathrm{x}_{\mathrm{k}}+\Delta$. Neste caso, o ciclo deverá ser percorrido no sentido do arco $e_{\mathrm{k}}$.

Se o arco selecionado $e_{\mathrm{k}}$ tem fluxo $\mathrm{x}_{\mathrm{k}}=\mathrm{u}_{\mathrm{k}}$, ou seja, $e_{\mathrm{k}} \in \psi_{2}$, deve-se diminuir o fluxo sobre este arco, então $\mathrm{x}_{\mathrm{k}}=\mathrm{x}_{\mathrm{k}}-\Delta$. Portanto, o ciclo deverá ser percorrido no sentido contrário ao do arco $e_{\mathrm{k}}$. 
Portanto, em ambos os casos, o fluxo corrente sobre o arco não-básico é quem determina em que o "sentido" o ciclo formado deverá ser percorrido. Esse "sentido" é chamado de orientação do ciclo e será denotado por $\delta$.

Dessa forma, a alteração do fluxo sobre o arco não-básico pode ser dada por:

$$
\mathrm{x}_{\mathrm{k}}=\mathrm{x}_{\mathrm{k}}+\Delta \delta \text {, onde } \delta=\left\{\begin{array}{lll}
1 & \text { se } & e_{\mathrm{k}} \in \Psi_{1} \\
-1 & \text { se } & e_{\mathrm{k}} \in \Psi_{2}
\end{array}\right. \text { é a orientação do ciclo. }
$$

Analisemos agora, quais os coeficientes de perturbação para os arcos básicos. Seja B uma base correspondente a árvore básica $7_{\mathrm{B}}$. Considerando um fluxo igual a $\Delta$ ou $\mathrm{u}_{\mathrm{k}}-\Delta$ sobre um arco não-básico $e_{\mathrm{k}}$, os coeficientes de perturbação dos fluxos sobre os arcos básicos são dados pelo vetor y onde:

$$
\mathbf{y}=\mathbf{B}^{-1} \mathbf{N}^{\mathrm{k}} \text {, onde } \mathbf{N}^{\mathrm{k}} \text { é a coluna não-básica correspondente ao arco } e_{\mathrm{k}} \text {. }
$$

Como $\mathbf{B}$ é triangular, y pode ser obtido diretamente por substituições recursivas, observando que:

$$
\mathbf{y}=\mathbf{B}^{-1} \mathbf{N}^{\mathrm{k}} \Rightarrow \mathbf{B y}=\mathrm{e}^{\mathrm{F}(\mathrm{k})}-\mathrm{e}^{\mathrm{T}(\mathrm{k})} \text {. }
$$

Novamente, como na determinação dos potenciais dos nós, a árvore básica $7_{\mathrm{B}}$ pode ser usada para obter os coeficientes do vetor direção $\mathbf{y}$.

Seja $\mathrm{P}=\left\{s_{1}, e_{\mathrm{j}_{1}}, s_{2}, e_{\mathrm{j}_{2}}, \ldots, s_{\mathrm{n}}, e_{\mathrm{j}_{\mathrm{n}}}, s_{\mathrm{n}+1}\right\}$ o único caminho na árvore básica $7_{\mathrm{B}}$ ligando nó $\mathrm{F}(\mathrm{k})$ ao nó $\mathrm{T}(\mathrm{k})$. Pela proposição A.5, tem-se que:

$$
\sum_{\mathrm{i}=1}^{\mathrm{n}} \mathbf{O}_{\mathrm{j}_{\mathrm{i}}}(\mathrm{P}) \mathbf{A}^{\mathrm{j}_{\mathrm{i}}}=\mathbf{e}^{\mathrm{F}(\mathbf{k})}-\mathbf{e}^{\mathrm{T}(\mathrm{k})},
$$

onde $\mathbf{A}^{\mathrm{j}_{\mathrm{i}}}$ são as colunas da base $\mathbf{B}$ correspondentes aos arcos pertencentes a árvore básica $\mathrm{e}$ $\mathrm{O}_{\mathrm{j}_{\mathrm{i}}}(\mathrm{P})$ é a orientação do arco $e_{\mathrm{j}_{\mathrm{i}}}$ no caminho.

Como as colunas $\mathbf{A}^{\mathrm{j}_{\mathrm{i}}}$ pertencentes a base $\mathbf{B}$ são linearmente independentes, a coluna nãobásica $\mathbf{N}^{\mathrm{k}}$, correspondente ao arco não-básico $e_{\mathrm{k}}$ selecionado, se escreve como combinação linear única das colunas $\mathbf{A}^{\mathrm{j}_{\mathrm{i}}}$ da base $\mathbf{B}$. Logo, as $m$ componentes de $\mathbf{y}$ podem ser determinadas pela seqüência de orientação do único caminho na árvore $7_{\mathrm{B}}$ ligando o nó $\mathrm{F}(\mathrm{k})$ ao nó $\mathrm{T}(\mathrm{k})$.

Fazendo todos os arcos em $7_{\mathrm{B}}$ serem ordenados como segue: $e_{\mathrm{j}_{1}}, e_{\mathrm{j}_{2}}, \ldots, e_{\mathrm{j}_{\mathrm{m}}}$ correspondendo as $m$ colunas de $\mathbf{B}$ tem-se:

$$
\mathrm{y}_{\mathrm{r}}=\left\{\begin{array}{ll}
\mathrm{O}_{\mathrm{j}_{\mathrm{i}}}(\mathrm{P}) & \text { se } e_{\mathrm{j}_{\mathrm{r}}}=e_{\mathrm{j}_{\mathrm{i}}} \in \mathrm{P} \\
\hat{\mathrm{u}} & \text { caso contrario }
\end{array} \mathrm{r}=1, \ldots, m .\right.
$$

Note que, para os arcos que pertencem a árvore geradora, mas não estão no caminho que liga o nó $\mathrm{F}(\mathrm{k})$ ao nó $\mathrm{T}(\mathrm{k})$ a componente correspondente de y é nula. Portanto, serão alterados somente os fluxos sobre os arcos ao longo do ciclo formado, os demais da árvore básica permanecerá os mesmos. 
A sequência de orientação do único caminho na árvore básica é sempre traçada a partir do nó $\mathrm{F}(\mathrm{k})$ para o nó $\mathrm{T}(\mathrm{k})$. No entanto, a alteração nos fluxos sobre os arcos básicos é feita quando o ciclo formado é percorrido segundo uma orientação determinada pelo fluxo corrente sobre o arco não-básico selecionado.

Portanto, os coeficientes da direção de perturbação dos arcos básicos no ciclo devem ser determinados pela seqüência de orientação dos arcos no único caminho que liga o nó $F(k)$ ao nó $\mathrm{T}(\mathrm{k})$, mas levando-se também em consideração o sentido em que o ciclo é percorrido.

Considere a figura dada a seguir:

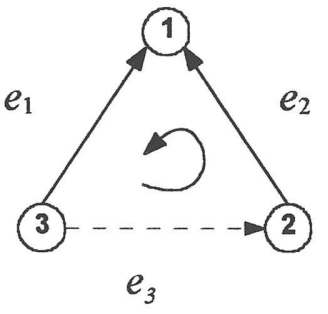

Figura 1.2a: $\mathrm{x}_{3}=0, \delta=1$

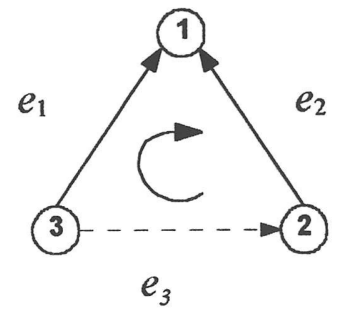

Figura 1.2b: $x_{3}=u_{3}, \delta=-1$.

Nas figuras $1.2 \mathrm{a}$ e $1.2 \mathrm{~b}$ acima, o arco selecionado para entrar na árvore básica é $e_{3} . \mathrm{O}$ único caminho que liga o nó $\mathrm{F}(3)=3$ ao nó $\mathrm{T}(3)=2$ é dado por $\mathrm{P}=\left\{3, e_{1}, 1, e_{2}, 2\right\}$, cuja seqüência de orientação é dada por $\mathrm{O}(\mathrm{P})=\{1,-1\}$.

Observe que, alterar os fluxos nos arcos da árvore básica ao longo do ciclo formado pela adição do arco não-básico $e_{3}$, é equivalente a aumentar o fluxo sobre o arco que está no mesmo sentido em que o ciclo é percorrido e diminuir o fluxo sobre o arco que está no sentido contrário daquele em que o ciclo é percorrido. Na figura 1.2a, onde $\delta=1$, o fluxo sobre o arco $e_{2}$ deve aumentar e sobre o arco $e_{1}$ deve diminuir. Já na figura $1.2 \mathrm{~b}$, onde $\delta=-1$, o fluxo sobre o arco $e_{1}$ deve aumentar, enquanto que sobre o arco $e_{2}$ deve diminuir.

Logo, a alteração dos fluxos sobre os arcos básicos pertencentes ao ciclo é dada por:

$$
\mathrm{x}_{\mathrm{j}}=\mathrm{x}_{\mathrm{j}}-\Delta \delta \mathrm{O}_{\mathrm{j}}(\mathrm{P}) \text {. }
$$

\subsubsection{Determinação de $\Delta$ (o valor da alteração dos fluxos)}

O fluxo sobre o arco não-básico $e_{\mathbf{k}}$ é alterado por $\Delta$ unidades para que a solução do problema de minimização seja melhorada, isto é, a função objetivo tenha um valor menor, o qual será melhor do que o valor que a solução corrente oferece.

Nesta seção será determinada por quantas unidades os fluxos sobre os arcos no ciclo deverão ser alteradas para obter uma nova solução que melhore o valor da função objetivo do problema. 
O limitante para o crescimento de $\Delta$ é obtido através das restrições de capacidade de fluxo, $0 \leq \mathrm{x}_{\mathrm{j}} \leq \mathrm{u}_{\mathrm{j}}$ para todo arco $e_{\mathrm{j}}$ no ciclo. Ou seja , pode-se alterar o fluxo sobre os arcos no ciclo formado até que um dos arcos no ciclo, atinja seu limite superior ou tenha valor nulo (limite inferior).

Portanto, deve-se verificar os seguintes casos:

- Se $\mathrm{O}_{\mathrm{j}}(\mathrm{P})=\delta$, isto é, a orientação do arco $e_{\mathrm{j}}$ é igual a orientação do ciclo, tem-se: $\mathrm{x}_{\mathrm{j}}=\mathrm{x}_{\mathrm{j}}-\Delta$, então deve-se garantir que $\mathrm{x}_{\mathrm{j}}-\Delta \geq 0 \Rightarrow \Delta \leq \mathrm{x}_{\mathrm{j}}$.

Portanto, neste caso $\Delta$ de ser escolhido como: $\Delta_{1}=\min _{O_{j}(P)=\delta}\left\{x_{j}\right\}$.

- $\mathrm{Se}-\mathrm{O}_{\mathrm{j}}(\mathrm{P})=\delta$, isto é, a orientação do arco $e_{\mathrm{j}}$ é contrária a orientação do ciclo, tem-se: $\mathrm{x}_{\mathrm{j}}=\mathrm{x}_{\mathrm{j}}+\Delta$, então deve-se garantir que $\mathrm{x}_{\mathrm{j}}+\Delta \leq \mathrm{u}_{\mathrm{j}} \Rightarrow \Delta \leq \mathrm{u}_{\mathrm{j}}-\mathrm{x}_{\mathrm{j}}$.

Logo, $\Delta$ deveria ser escolhido como sendo: $\Delta_{2}=\min _{-O_{j}(P)=\delta}\left\{u_{j}-x_{j}\right\}$.

- Além de certificar-se da validade das restrições de capacidade para os fluxo dos arcos básicos no ciclo, também deve ser verificada a validade da mesma restrição para a variável não-básica entrando na árvore.

$O$ arco não-básico é alterado por $\mathrm{x}_{\mathrm{k}}=\mathrm{x}_{\mathrm{k}}+\Delta \delta$, onde :

$$
\begin{aligned}
& \text { se } x_{k}=0 \Rightarrow \delta=1, \quad x_{k}=0+\Delta \leq u_{k} \Rightarrow \Delta \leq u_{k} \\
& \text { se } x_{k}=u_{k} \Rightarrow \delta=-1, \quad x_{k}=u_{k}-\Delta \leq u_{k} \Rightarrow \Delta \leq u_{k}
\end{aligned}
$$

A alteração $\Delta$ que os fluxos sofrerão, deve ser escolhida de forma a satisfazer simultaneamente as restrições de capacidade para todos os arcos no ciclo formado. Assim, $\Delta$ é escolhido como sendo:

$$
\Delta=\min \left\{\Delta_{1}, \Delta_{2}, u_{k}\right\} .
$$

Se $\Delta=\Delta_{1}$, então um arco básico atingiu o seu limite inferior.

Se $\Delta=\Delta_{2}$, então um arco básico atingiu o seu limite superior.

Se $\Delta=u_{\mathrm{k}}$, então o arco não-básico entrando na árvore básica atingiu o seu limite oposto antes que qualquer outro arco básico atingisse um dos seus limites. Quando isto acontece, nenhuma mudança é feita na base B. Portanto, depois de atualizados os valores dos fluxos sobre os arcos básicos e o arco não-básico por $\Delta$, o arco $e_{\mathrm{k}}$ deve ser trocado nos conjuntos $\psi_{1}$ e $\psi_{2}$. Feito isso, a condição de otimalidade é analisada novamente. Se ela estiver sendo satisfeita o algoritmo termina, caso contrário um novo arco não-básico deverá ser selecionado para entrar na árvore básica. 


\subsubsection{Algoritmo simplex sobre uma rede}

\section{Inicialização:}

Seja $\eta_{\mathbf{B}}$ uma árvore básica enraizada factível e $\mathrm{x}_{\mathrm{j}}$ o fluxo sobre o arco $e_{\mathrm{j}} \in \overline{7}_{\mathbf{B}}$.

Calcule $\pi$ pelo algoritmo 1.7.2 .

1. Teste da otimalidade:

Faça $\psi_{1}=\left\{e_{\mathrm{k}} ; \mathrm{x}_{\mathrm{k}}=0\right.$ e $\left.c_{\mathrm{k}}-\pi_{\mathrm{F}(\mathrm{k})}+\pi_{\mathrm{T}(\mathrm{k})}<0\right\}$

$$
\psi_{2}=\left\{e_{\mathrm{k}} ; \mathrm{x}_{\mathrm{k}}=\mathrm{u}_{\mathrm{k}} \text { e } \mathrm{c}_{\mathrm{k}}-\pi_{\mathrm{F}(\mathrm{k})}+\pi_{\mathrm{T}(\mathrm{k})}>0\right\} .
$$

Se $\psi_{1} \cup \psi_{2}=\varnothing$, termine solução corrente é ótima.

Se não selecione $e_{\mathrm{k}} \in \psi_{1} \cup \psi_{2}$.

Faça $\delta=\left\{\begin{array}{ccc}1 & \text { se } & e_{\mathrm{k}} \in \psi_{1} \\ -1 & \text { se } & e_{\mathrm{k}} \in \psi_{2}\end{array}\right.$

2. Determinação de $\Delta$ :

Seja $\mathrm{P}=\left\{s_{1}, e_{\mathrm{j}_{1}}, s_{2}, e_{\mathrm{j}_{2}}, \ldots, s_{\mathrm{n}}, e_{\mathrm{j}_{\mathrm{n}}}, s_{\mathrm{n}+1}\right\}$ o caminho em $7_{\mathrm{B}}$ ligando $\mathrm{F}(\mathrm{k})$ a $\mathrm{T}(\mathrm{k})$.

Faça $\Delta_{1}=\min _{O_{j}(P)=\delta}\left\{x_{j}\right\}, \quad \Delta_{2}=\min _{O_{j}(P)=-\delta}\left\{u_{j}-x_{j}\right\}$ e $\Delta=\min \left\{\Delta_{1}, \Delta_{2}, u_{k}\right\}$.

3. Atualização dos fluxos:

Faça $x_{k}=x_{k}+\Delta \delta$

Para todo $e_{\mathrm{j}} \in \mathrm{P}$ faça $\mathrm{x}_{\mathrm{j}}=\mathrm{x}_{\mathrm{j}}-\Delta \delta \mathrm{O}_{\mathrm{j}}(\mathrm{P})$.

Se $\Delta=u_{k}$, retorne ao passo 1 .

4. Atualização da árvore e dos potenciais dos nós:

Faça $\quad \psi_{3}=\left\{e_{\mathrm{j}} ; \mathrm{x}_{\mathrm{j}}=0\right.$ onde $\left.\mathrm{O}_{\mathrm{j}}(\mathrm{P})=\delta\right\} \mathrm{e}$

$$
\psi_{4}=\left\{e_{j} ; \mathrm{x}_{\mathrm{j}}=\mathrm{u}_{\mathrm{j}} \text { onde }-\mathrm{O}_{\mathrm{j}}(\mathrm{P})=\delta\right\} .
$$

Selecione $e_{\mathrm{s}} \in \psi_{3} \cup \psi_{4}$ e substitua $e_{\mathrm{s}}$ na árvore básica $7_{\mathrm{B}}$ por $e_{\mathrm{k}}$.

Atualize os potenciais dos nós.

Retorne ao passo 1.

\section{8 Árvore geradora inicial}

No algoritmo simplex anterior, foi assumido que uma árvore básica geradora factível para iniciar o algoritmo estava disponível.

Existem várias estratégias para encontrar uma solução básica factível. A apresentada aqui é chamada início com todas artificiais e envolve a ampliação da rede.

Considere a matriz incidência nó-arco sem o arco raiz adicionado. Suponhamos que a rede associada tenha $m$ nós.

Primeiramente, é acrescentado um novo nó $m+1$. A seguir, para cada nó estoque $i$ com estoque $b_{i}$ acrescentamos um arco artificial a partir do nó $i$ para o nó $m+1$, tendo custo e limite 
iguais a infinito. O fluxo sobre este arco será igual a $b_{i}$, satisfazendo desta maneira a restrição de conservação de fluxo do nó $i$.

Para cada nó demanda $t$, com demanda $\left|b_{\mathfrak{t}}\right|$, acrescentamos um arco artificial a partir do nó $m+1$ para o nó $t$ tendo custo e limite iguais a infinito. $O$ fluxo sobre este arco será $\left|b_{t}\right|$, satisfazendo assim a restrição de conservação de fluxo do nó $t$.

Desde que os custos associados com os arcos artificiais são infinitos, qualquer solução sem fluxo sobre os arcos artificiais é melhor que uma solução tendo fluxo sobre estes arcos. Este método corresponde ao Método do M-Grande.

A árvore básica é completada usando arcos originais, cada um com fluxo igual a zero. Um exemplo do método início com todas artificiais é ilustrado na figura a seguir.

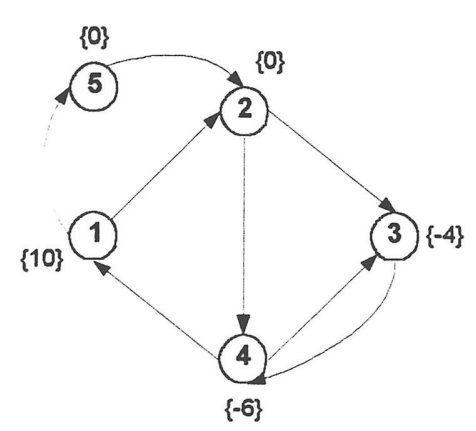

Figura 1.3a: Rede original

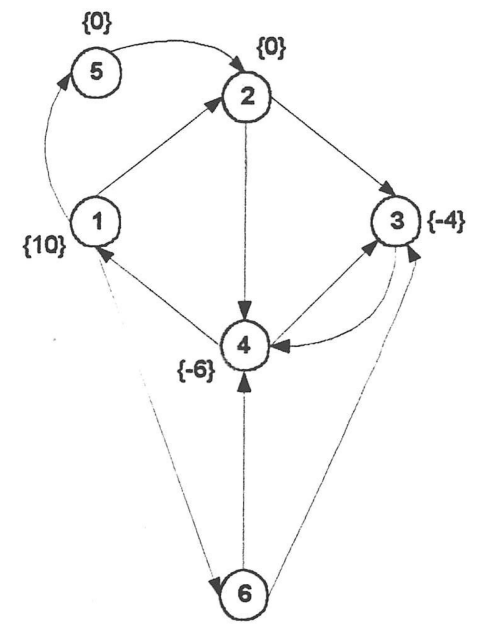

Figura 1.3b: Rede transformada

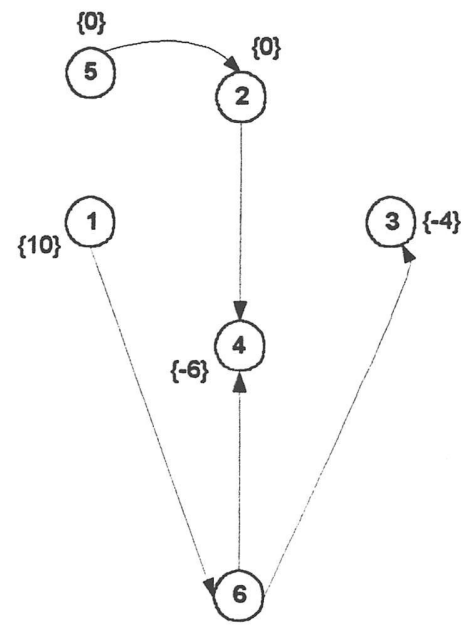

Figura 1.3c: Árvore geradora inicial

\subsection{Uma regra para evitar a ciclagem}

O algoritmo simplex para redes, como descrito anteriormente, mantém uma árvore básica factível a cada iteração, até obter uma estrutura árvore geradora $([\mathbf{B}, \mathbf{N}])$ que satisfaça a condição de otimalidade (proposição 1.8).

Se a cada iteração, a árvore básica é não-degenerada, o algoritmo simplex terminará em um número finito de iterações. Pois neste caso, a alteração $\Delta$ será sempre positiva $(\Delta>0)$, permitindo que o custo da nova estrutura árvore geradora seja $\Delta\left|c_{j}^{\pi}\right|$ unidades menor do que a estrutura árvore geradora anterior, isto é a cada iteração a função objetivo do problema sofierá um decréscimo de $\Delta\left|c_{j}^{\pi}\right|$ unidades. E como o número de árvores básicas é finito e cada uma das estruturas árvores geradoras tem um único custo associado, o algoritmo encontrará qualquer uma destas estruturas no máximo uma vez, terminando portanto, em um número finito de iterações. 
Entretanto, se em uma iteração a árvore é não-degenerada, isto não significa que em nenhum momento não ocorrerá uma árvore degenerada. O algoritmo simplex pode obter uma árvore degenerada a partir de uma árvore não-degenerada, quando dois ou mais arcos são candidatos a sair desta árvore básica no passo 4.

Diante da degeneração o algoritmo simplex não necessariamente termina em um número finito de iterações. Pois, se uma árvore básica é degenerada, na iteração seguinte o $\Delta$ escolhido será nulo $(\Delta=0)$. Depois de realizar a troca de arcos na árvore básica no passo 4 , uma nova árvore básica é obtida. Entretanto, essa nova árvore corresponderá a mesma solução anterior, já que os fluxos não sofreram nenhuma alteração. Logo, nenhum avanço na direção da otimalidade fosse feito.

Isto poderia fazer com que partições básicas fossem repetidas indefinidamente, cada uma tendo $\Delta=0$. Este fenômeno ao qual o método simplex está sujeito diante da degeneração é chamado ciclagem.

Para que o método termine em um número finito de iterações, algumas restrições sobre a escolha da variável saindo da base são impostas.

O algoritmo simplex mantém uma árvore geradora a cada iteração. Mantendo um tipo especial de árvore geradora, chamada árvore geradora fortemente factível, o algoritmo simplex termina em um número finito de iterações mesmo quando a árvore geradora é degenerada.

Uma árvore geradora é dita ser fortemente factível se todo arco $e_{\mathrm{j}}$ na árvore básica com fluxo $\mathrm{x}_{\mathrm{j}}=0$ está orientado para o nó raiz, enquanto que todo arco $e_{\mathrm{j}}$ cujo fluxo $\mathrm{x}_{\mathrm{j}}=\mathrm{u}_{\mathrm{j}}$ está orientado para fora do nó raiz. (Toda árvore não-degenerada é fortemente factível).

Seja $\mathrm{P}$ o único caminho o único caminho na árvore básica inicial a partir do nó $i$ para o nó raiz. Começando pelo nó $\mathrm{F}(\mathrm{k})$ traça-se o caminho a partir deste nó para o nó raiz, marcando todos os nós deste caminho. Em seguida traça-se o caminho do nó $\mathrm{T}(\mathrm{k})$ para o nó raiz. $\mathrm{O}$ primeiro nó em comum entre esses dois caminhos, é chamado apex.

Regra para evitar a ciclagem: Selecione o arco saindo como sendo o último arco encontrado, candidato a sair da árvore, quando o ciclo é percorrido ao longo de sua orientação começando pelo nó apex. 


\section{Capítulo 2}

\section{Multifluxo em Redes}

\subsection{Introdução}

No capítulo anterior os problemas de fluxo em redes eram compostos por um único produto, o qual desejava-se enviar pela rede, a partir de certos nós estoques para seus nós demandas, de modo que o custo total ou caminho percorrido fosse mínimo. Entretanto, existem problemas onde mais de um produto compartilham a mesma rede, tais problemas são chamados de Problemas de Multifluxo em redes.

Problemas de multifluxo em redes possuem uma característica estrutural importante, podendo ser representados, em parte, como um conjunto de subproblemas separáveis, agregados por um número de restrições adicionais. Número este, menor do que o número total de restrições imposto sobre o problemas. Problemas deste tipo que aparecem na prática, frequentemente, possuem um número extremamente grande de variáveis e restrições. Sendo assim, a aplicação direta do método simplex para obter uma solução, pode não ser recomendável.

Existem duas abordagens básicas e atrativas, que foram e estão sendo utilizadas pelos pesquisadores no desenvolvimento de técnicas especializadas para resolverem problemas deste tipo: - Particionamento e a Decomposição.

Neste capítulo apresentaremos uma formulação matemática para o problema de multifluxo e a seguir a abordagem do particionamento primal, o qual é uma especialização do método simplex.

\subsection{Definição do Problema de Multifluxo em Redes}

Existe uma classe de problemas de fluxo em redes chamada Multifluxo em Redes ou Fluxo em Redes de Multiprodutos, na qual é necessário diferenciar os fluxos de vários produtos na rede.

$\mathrm{O}$ que caracteriza um problema de multifluxo em rede, é o fato de produtos distintos compartilharem os mesmos recursos disponíveis na rede. Portanto, para encontrar o fluxo ótimo destes produtos na rede, é necessário resolver os problemas em comum acordo com cada outro. 
$\mathrm{Na}$ formulação matemática, essa interação entre os problemas, está representado pelas chamadas restrições de acoplamento ou restrições de capacidade mútua, que limitam os fluxos dos produtos sobre alguns ou todos os recursos disponíveis na rede. Se os produtos não interagirem de alguma maneira, isto é, não compartilham nenhum dos recursos existentes limitados, os fluxos destes produtos podem ser vistos como problemas de fluxo em rede independentes.

O termo produto está sendo usado como um item específico, isto é, uma mercadoria que flui pela rede, sendo diferenciado apenas pelas suas características físicas. Por exemplo, em uma rede onde deseja-se transportar milho e arroz, pela definição usada presentemente tem-se somente dois produtos. Essa definição para produto implica em uma formulação para o problema de multifluxo chamada Produto Específico, formulação esta apresentada a seguir e que será utilizada durante o decorrer deste capítulo. Entretanto, os produtos podem ser diferenciados não somente pelas suas características físicas, mas também pelos seus pares origem e destino.

No capítulo seguinte, será apresentada outras duas definições para produto, de acordo com seus pares origem e destino, as quais implicarão em outras duas formulações para o problema de multifluxo.

A rede é assumida ser idêntica para todos os produtos.

\subsection{Formulação do Problema}

Seja $\mathcal{G}$ uma rede com $m$ nós e $n$ arcos na qual existe um fluxo de $\mathrm{K}$ produtos distintos. A formulação matemática, em notação matricial, para o problema de multifluxo em rede pode ser apresentado como:

$$
\begin{array}{ll}
\text { Minimizar } & \sum_{\mathbf{k}=1}^{K} \mathbf{c}^{\mathrm{k}} \mathbf{x}^{\mathrm{k}} \\
\text { sujeito a } & \mathbf{A \mathbf { x } ^ { \mathrm { k } }}=\mathbf{b}^{\mathrm{k}} \\
& \sum_{\mathbf{k}=1}^{\mathrm{K}} \mathbf{D}^{\mathrm{k}} \mathbf{x}^{\mathrm{k}} \leq \mathbf{r} \\
& \mathbf{0} \leq \mathbf{x}^{\mathrm{k}} \leq \mathbf{u}^{\mathrm{k}} \quad \mathrm{k}=1, \ldots, \mathrm{K}
\end{array}
$$

onde: A é a matriz incidência nó-arco $(m \times n)$ para a rede $\mathscr{G}$ (definida no apêndice A);

$\mathbf{b}^{\mathrm{k}}$ é o vetor estoque/demanda para cada produto $\mathrm{k}$, com $m$ componentes (número de nós). Será assumido que a rede está balanceada, isto é, o estoque total é igual a demanda total, $\mathbf{1 b}^{\mathbf{k}}=0$ $\mathrm{k}=1, \ldots, \mathrm{K}$;

$\mathrm{c}^{\mathrm{k}}$ é o vetor custo do produto $\mathrm{k}$, com $n$ componentes (número de arcos) e $\mathrm{c}_{\mathrm{j}}^{\mathrm{k}}$ é o custo do produto k sobre o $\operatorname{arco} e_{\mathrm{j}}$;

$\mathbf{u}^{\mathrm{k}}$ é o vetor de capacidade individual do arco para cada produto $\mathrm{k}$, com $n$ componentes; 
$\mathbf{x}^{\mathrm{k}}$ é o vetor fluxo para cada produto $\mathrm{k}$ na rede, variável de decisão com $n$ componentes e $\mathrm{x}_{\mathbf{j}}^{\mathrm{k}}$ é a variável fluxo sobre o arco $e_{\mathrm{j}}$;

$\mathbf{D}^{\mathbf{k}}$ é uma matriz diagonal $(n \times n)$ e $\forall \mathbf{k} \mathbf{D}_{\mathrm{jj}}{ }^{\mathrm{k}}= \begin{cases}1 & \text { se o arco } e_{\mathrm{j}} \text { tem capacidade mutua } \\ 0 & \text { caso contrario }\end{cases}$ $\mathrm{D}_{\mathrm{jj}}^{\mathrm{k}}$ também pode ser visto como um "fator peso" com relação a essa restrição;

r é o vetor de capacidade mútua sobre os fluxos nos arcos com $n$ componentes.

A restrição $2.1 \mathrm{~b}$ é uma coleção de $\mathrm{K}$ conjuntos de restrições de conservação de fluxo, modelando o fluxo sobre a rede para cada produto; $2.1 \mathrm{~d}$ são as restrições de capacidade de fluxo individual de cada produto $\mathrm{k}$, sobre cada arco. Em alguns problemas tem-se $\mathrm{u}_{\mathrm{j}}^{\mathrm{k}}=\infty$, isto é, o fluxo sobre o arco $e_{\mathrm{j}}$ para algum produto $\mathrm{k}$ não é limitado, ou até mesmo para todo produto $\mathrm{k}$ e/ou todo $\operatorname{arco} e_{\mathrm{j}}$. Quando isto acontece, tem-se somente as restrições de não-negatividade, ou seja, $\mathbf{x}^{\mathbf{k}} \geq \mathbf{0}$, para todo produto k; as restrições $2.1 \mathrm{c}$ são as restrições de acoplamento ou capacidade mútua que restringem o fluxo total (de todos os produtos), sobre cada arco $e_{\mathrm{j}}$. São estas as restrições que "amarram", ou melhor, agregam os problemas de fluxo de todos os produtos, transformando-os em um único problema, de dimensão maior, o qual é chamado de problema de multifluxo em rede. Os problemas de fluxos em rede para todos os produtos agora devem ser resolvidos, de maneira que satisfaçam as restrições de acoplamento.

A matriz de restrições para o problema de multifluxo pode ser representada por:

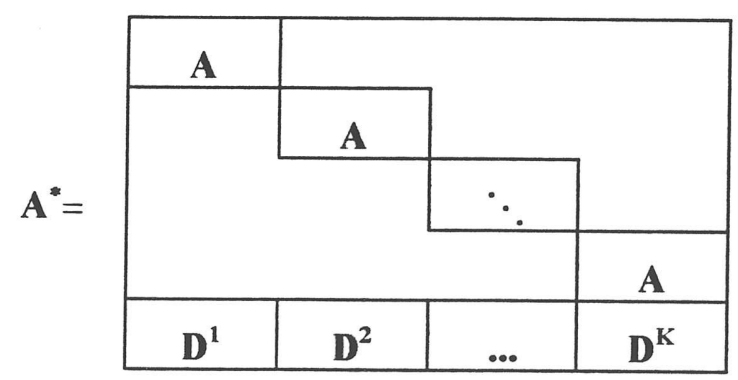

Figura 2.1 - Matriz de restrições para o Problema de Multifluxo

\subsection{Particionamento}

A abordagem Particionamento explora a estrutura bloco angular com restrições adicionais da matriz do problema de multifluxo (ver figura 2.1), particionando a matriz básica, de maneira que, partes destas tenham características das bases individuais de cada um dos produtos na rede.

Os métodos que utilizam a abordagem de particionanıento são especializações do método simplex, entre eles estão: o Particionamento Primal, o Particionamento Dual e o Particionamento Primal-Dual. Neste trabalho apresentaremos o Particionamento Primal.

[Hartman/Lasdon-72] apresentaram o método Generalized Upper Bounding (GUB) para problemas de multifluxo em redes, usando a formulação baseada em fluxos nos arcos. Este método é 
uma especialização do Generalized Upper Bounding para problemas blocos angulares apresentado por [Lasdon-72]. [Helgason/Kennington-77] apresentaram os detalhes da implementação do método de particionamento primal de [Hartman/Lasdon-72].

Daremos a seguir, alguns resultados algébricos que serão úteis na apresentação do método de particionamento primal por [Helgason/Kennington-80].

\subsubsection{Resultados algébricos}

Considere a seguinte matriz $\mathbf{E}$ da forma:

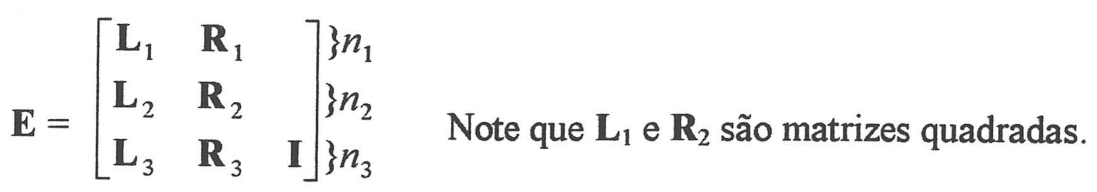

$$
\begin{aligned}
& n_{1} n_{2} \quad n_{3}
\end{aligned}
$$

Primeiramente, examinaremos alguns resultados importantes de operações executadas com a matriz $\mathbf{E}$ dada acima, pois estas operações são requeridas pelo método simplex. Uma delas é $\pi \mathbf{E}=$ c, que determinam as variáveis duais, utilizadas para calcular os custos reduzidos, e estes por sua vez determinam qual a variável não-básica a entrar na base; a outra é Ey = a, que determina qual a direção de perturbação das variáveis básicas, para a obtenção dos novos valores para as mesmas.

Proposição 2.1: Se $\operatorname{det}\left(\mathbf{L}_{1}\right) \neq 0$ e $\operatorname{det}(\mathbf{E}) \neq 0$, então $\operatorname{det}\left(\mathbf{R}_{2}-\mathbf{L}_{2} \mathbf{L}_{1}{ }^{-1} \mathbf{R}_{1}\right) \neq 0$.

demonstração: Seja

$$
\begin{gathered}
\mathbf{T}_{1}=\left[\begin{array}{ccc}
\mathbf{I} & -\mathbf{L}_{1}^{-1} \mathbf{R}_{1} & \\
& \mathbf{I} &
\end{array}\right] \quad \mathbf{T}_{2}=\left[\begin{array}{ccc}
\mathbf{I} & & \\
& \mathbf{I} & \\
& \mathbf{L}_{3} \mathbf{L}_{1}^{-1} \mathbf{R}_{1}-\mathbf{R}_{3} & \mathbf{I}
\end{array}\right] \text {, onde } \operatorname{det}\left(\mathrm{T}_{1}\right)=\operatorname{det}\left(\mathrm{T}_{2}\right)=1 . \\
\text { Considere } \left.\mathrm{ET}_{1} \mathbf{T}_{2}=\left[\begin{array}{ccc}
\mathbf{L}_{1} & & \\
\mathbf{L}_{2} & \mathbf{R}_{2}-\mathbf{L}_{2} \mathbf{L}_{1}^{-1} \mathbf{R}_{1} & \\
\mathbf{L}_{3} & & \mathbf{I}
\end{array}\right]\right\} n_{2} \text {. Então } \operatorname{det}\left(\mathrm{ET}_{1} \mathrm{~T}_{2}\right) \neq 0 \text { implica que } \mathrm{ET}_{1} \mathbf{T}_{2}
\end{gathered}
$$

tem posto coluna completo, então $\mathbf{R}_{2}-\mathbf{L}_{2} \mathbf{L}_{1}{ }^{-1} \mathbf{R}_{1}$ deve ter posto coluna completo, caso contrário $\mathrm{ET}_{1} \mathrm{~T}_{2}$ não teria posto coluna completo. Portanto $\operatorname{det}\left(\mathbf{R}_{2}-\mathbf{L}_{2} \mathbf{L}_{1}{ }^{-1} \mathbf{R}_{1}\right) \neq 0$.

Proposição 2.2: Se $\operatorname{det}\left(\mathbf{L}_{1}\right) \neq 0 \operatorname{edet}(\mathbf{E}) \neq 0$, a solução para

$$
\left[\begin{array}{lll}
\mathbf{L}_{1} & \mathbf{R}_{1} & \\
\mathbf{L}_{2} & \mathbf{R}_{2} \\
\mathbf{L}_{3} & \mathbf{R}_{3} & \mathbf{I}
\end{array}\right]\left[\begin{array}{l}
\mathbf{x} \\
\mathbf{y} \\
\mathbf{z}
\end{array}\right]=\left[\begin{array}{c}
\mathbf{u} \\
\mathbf{r} \\
\mathbf{w}
\end{array}\right]
$$

é dada por: $\quad \mathbf{y}=\left(\mathbf{R}_{2}-\mathbf{L}_{2} \mathbf{L}_{1}^{-1} \mathbf{R}_{1}\right)^{-1}\left(\mathbf{r}-\mathbf{L}_{2} \mathbf{L}_{1}^{-1} \mathbf{u}\right)$

$$
\begin{aligned}
& \mathbf{x}=\mathbf{L}_{1}^{-1} \mathbf{u}-\mathbf{L}_{1}^{-1} \mathbf{R}_{1} \mathbf{y} \\
& \mathbf{z}=\mathbf{w}-\mathbf{L}_{3} \mathbf{L}_{1}^{-1} \mathbf{u}-\left(\mathbf{R}_{3}-\mathbf{L}_{3} \mathbf{L}_{1}^{-1} \mathbf{R}_{1}\right) \mathbf{y} .
\end{aligned}
$$


Proposição 2.3: Se $\operatorname{det}\left(\mathbf{L}_{1}\right) \neq 0 \quad \operatorname{det}(\mathbf{E}) \neq 0$, a solução para

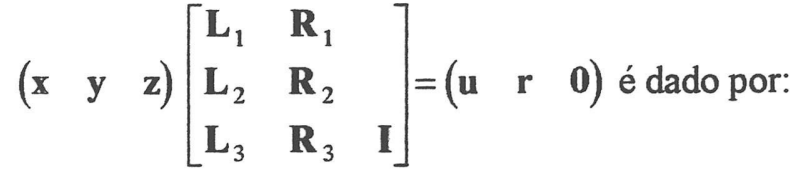

$$
\begin{aligned}
& \mathbf{z}=\mathbf{0} \\
& \mathbf{y}=\left(\mathbf{r}-\mathbf{u ~ L} \mathbf{L}_{1}^{-1} \mathbf{R}_{1}\right)\left(\mathbf{R}_{2}-\mathbf{L}_{2} \mathbf{L}_{1}^{-1} \mathbf{R}_{1}\right)^{-1} \\
& \mathbf{x}=\left(\mathbf{u}-\mathbf{y} \mathbf{L}_{2}\right) \mathbf{L}_{1}^{-1} \text {. }
\end{aligned}
$$

\subsubsection{Caracterização de base para o problema de multifluxo}

Considere uma rede $\mathfrak{q}=[\varkappa, \mathcal{A}]$ com $m$ nós e $n$ arcos. Para obter restrições de igualdade no problema 2.1, acrescentamos um vetor (s) de variáveis de folga. $\mathrm{E}$ antes de apresentar uma base para o problema de multifluxo, acrescentando o arco raiz $\left(a_{k}\right)$ na matriz incidência nó-arco de cada produto $\mathrm{k}$, obtem-se:

$$
\begin{array}{ll}
\text { Minimizar } & \sum_{\mathbf{k}} \mathbf{c}^{\mathrm{k}} \mathbf{x}^{\mathbf{k}} \\
\text { sujeito a } \quad & \mathbf{A \mathbf { x } ^ { \mathrm { k } }}+\mathbf{e}^{l} \mathbf{a}_{\mathbf{k}}=\mathbf{b}^{\mathbf{k}} \quad \forall \mathbf{k} \\
& \sum_{\mathbf{k}} \mathbf{D}^{\mathrm{k}} \mathbf{x}^{\mathbf{k}}+\mathbf{s}=\mathbf{r} \\
& \mathbf{0} \leq \mathbf{x}^{\mathbf{k}} \leq \mathbf{u}^{\mathbf{k}} \text { e } 0 \leq \mathbf{a}_{\mathbf{k}} \leq 0 \quad \forall \mathbf{k}, \mathbf{s} \geq \mathbf{0} .
\end{array}
$$

A matriz das restrições associada a este problema é dada por:

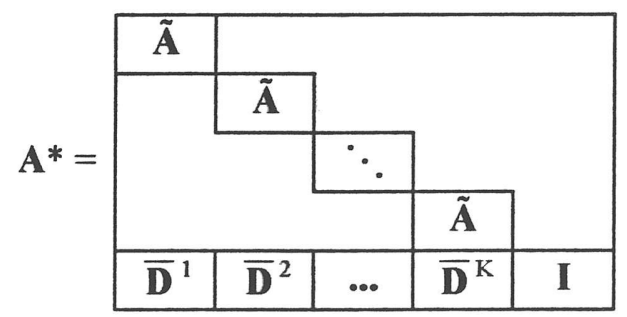

Figura 2.2 Matriz de restrições para o problema 2.2

onde $\tilde{\mathbf{A}}$ é a matriz incidência nó-arco acrescentada do arco raiz e $\overline{\mathbf{D}}^{\mathrm{k}}$ tem uma coluna nula devido ao acréscimo do arco raiz na matriz de incidência nó-arco.

Proposição 2.4. Cada base para $\mathbf{A}^{*}$ pode ser colocada na forma:

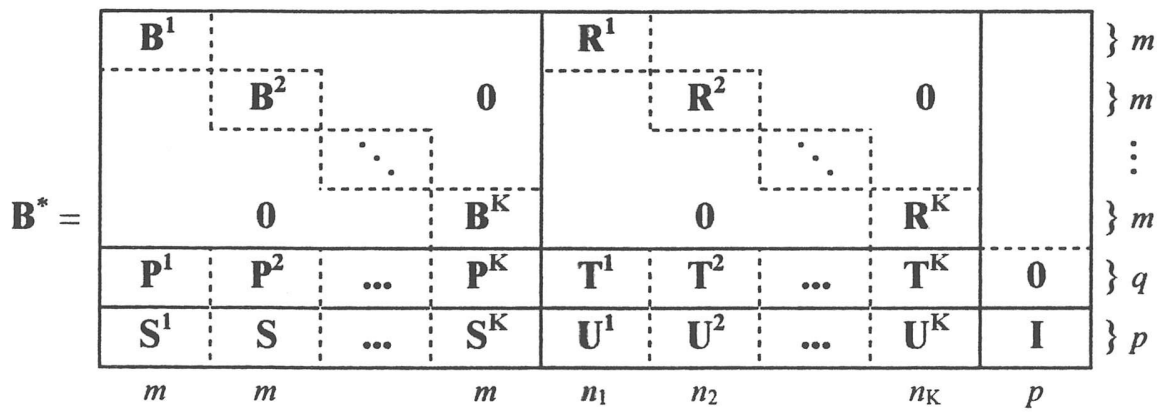

Figura 2.3 - Matriz básica para Problemas de Multifluxo

onde $\mathbf{B}^{1}, \ldots, \mathbf{B}^{\mathrm{K}}$ são as bases para as matrizes $\tilde{\mathbf{A}}$ de cada produto. 
Na matriz básica $\mathbf{B}^{*}$, existem $\mathrm{K}$ submatrizes $\mathbf{B}^{\mathrm{k}}(m \times m)$, que são bases para cada um dos produtos. Sendo assim, existem $\mathrm{Km}$ colunas na base $\mathbf{B}^{*}$, associadas com as bases dos produtos individuais e essas colunas são chamadas colunas chaves.

Para cada submatriz $\mathbf{B}^{\mathbf{k}}$ da base $\mathbf{B}^{*}$, tem-se duas outras submatrizes associadas $\mathbf{P}^{\mathbf{k}}(q \times m)$ e $\mathbf{S}^{\mathbf{k}}$ $(p \times m)$, que identificam os arcos nas restrições de acoplamento que estão na base individual $\mathbf{B}^{\mathrm{k}}$ do produto $\mathrm{k}$.

Considere a submatriz:

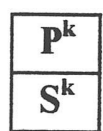

Essa submatriz possui uma coluna nula correspondente ao arco raiz em $\mathbf{B}^{\mathbf{k}}$ e vetores unitários para as $m-1$ colunas restantes. Se a componente de valor igual a " 1 " estiver entre as $q$ primeiras componentes, isto é, em $\mathbf{P}^{\mathrm{k}}$, o arco correspondente na base $\mathbf{B}^{*}$ é chamado saturado, pois está no limite de sua capacidade mútua. Logo, a variável de folga para a restrição de capacidade mútua relativo a este arco é nula. Se a componente com valor igual a "1" estiver entre as $p$ restantes, isto é em $\mathbf{S}^{\mathbf{k}}$, o arco associado na base $\mathbf{B}^{*}$ é chamado não-saturado (não está no limite de sua capacidade mútua) e a variável de folga na restrição de capacidade mútua em relação a este arco é diferente de zero. As variáveis de folga estão representadas pela submatriz identidade I $(p \times p)$.

Cada submatriz $\mathbf{R}^{\mathrm{k}}\left(m \times n_{\mathrm{k}}\right)$ que estão na base $\mathbf{B}^{*}$, contém colunas (arcos) que não pertencem a base individual $\left(\mathbf{B}^{\mathrm{k}}\right)$ do produto $\mathrm{k}$. Observe que, a submatriz $\mathbf{R}^{\mathrm{k}}$ pode ser nula para algum $\mathrm{k}$. Se isso acontecer, as submatrizes $\mathbf{T}^{\mathrm{k}}$ e $\mathbf{U}^{\mathrm{k}}$ correspondentes também serão nulas e consequentemente não pertencerão a base $\mathbf{B}^{*}$.

Todas as colunas associadas com as submatrizes $\mathbf{R}^{\mathbf{k}}$ (para todo $\mathrm{k}$ ) mais as colunas correspondentes da submatriz I, são chamadas colunas não-chaves.

Para cada submatriz $\mathbf{R}^{\mathrm{k}}$ em $\mathbf{B}^{*}$, existem outras duas submatrizes associadas, $\mathbf{T}^{\mathrm{k}}\left(q \times n_{\mathrm{k}}\right)$ e $\mathbf{U}^{\mathrm{k}}$ $\left(p \times n_{\mathrm{k}}\right)$ que identificam os arcos nas restrições de capacidade mútua que não pertencem a base individual $\mathbf{B}^{\mathbf{k}}$.

\begin{tabular}{ll|}
\hline & $\mathbf{T}^{\mathrm{k}}$ \\
\hline & $\mathbf{U}^{\mathrm{k}}$ \\
\hline
\end{tabular}

As colunas dessa submatriz também são vetores unitários, onde se a componente com valor igual a "l" estiver entre as $q$ primeiras componentes o arco é saturado, e se estiver entre as $p$ restantes o arco é não-saturado.

\subsubsection{A Matriz Ciclo}

Depois que a matriz básica está na forma como mostrada na figura 2.3 , a base $\mathbf{B}^{*}$ pode ser triangularizada multiplicando-a por uma matriz $\mathbf{T}$ dada por: 


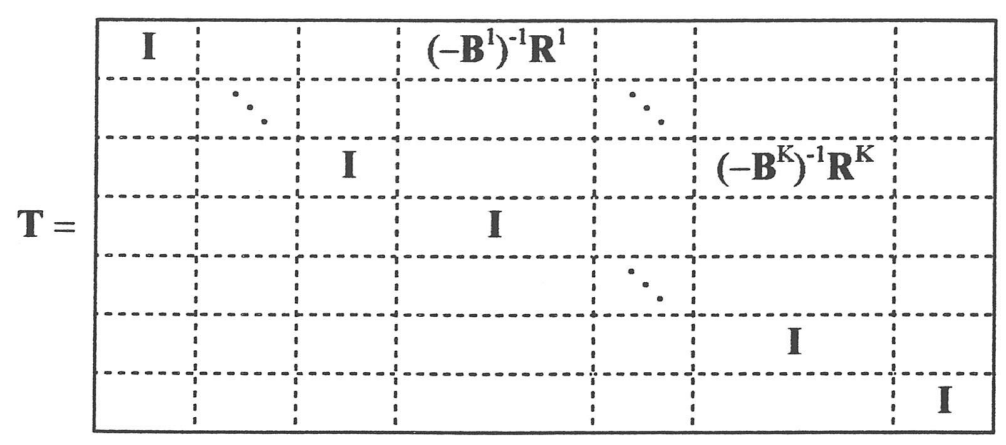

Obtem-se uma matriz básica bloco triangular inferior, da forma:

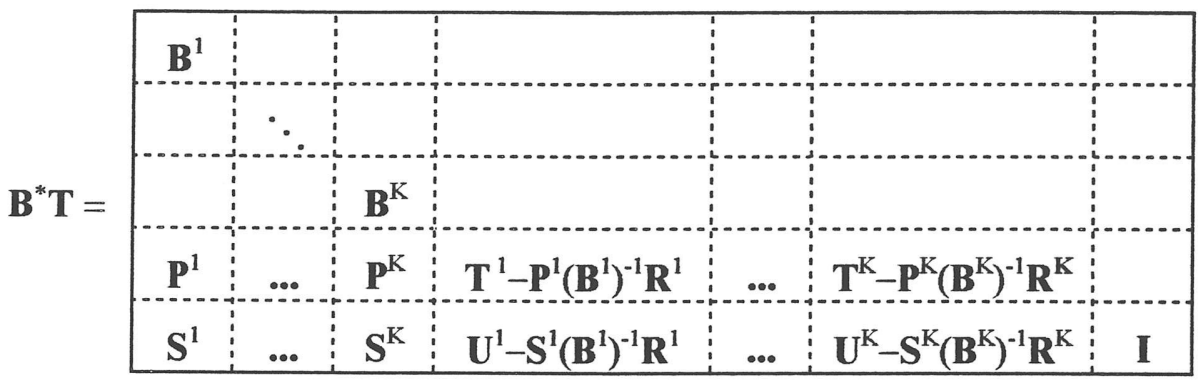

A matriz $\mathbf{R}_{2}-\mathbf{L}_{2} \mathbf{L}_{1}^{-1} \mathbf{R}_{1}$, proveniente da proposição 2.1 pela triangularização de $\mathbf{B}^{*}$, será denotada por $\mathbf{Q}$.

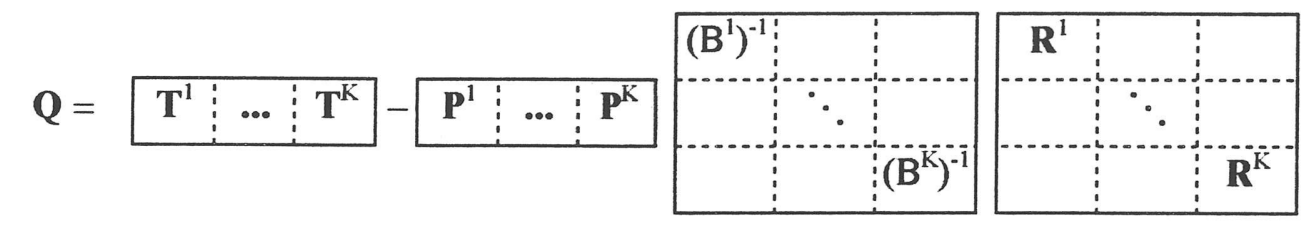

$\mathbf{Q}=\mathbf{R}_{2}-\mathbf{L}_{2} \mathbf{L}_{1}^{-1} \mathbf{R}_{1}=\left(\mathbf{T}^{1}-\mathbf{P}^{1}\left(\mathbf{B}^{1}\right)^{-1} \mathbf{R}^{1} \ldots \mathbf{T}^{\mathrm{K}}-\mathbf{P}^{\mathrm{K}}\left(\mathbf{B}^{\mathrm{K}}\right)^{-1} \mathbf{R}^{\mathrm{K}}\right)$.

Esta matriz é chamada base trabalho ou matriz ciclo. Uma coluna da matriz ciclo para algum produto $\mathrm{k}$, é dada por:

$$
\mathbf{T}_{\mathbf{j}}{ }^{\mathrm{k}}-\mathbf{P}^{\mathrm{k}}\left(\mathbf{B}^{\mathrm{k}}\right)^{-1} \mathbf{R}_{\mathbf{j}}^{\mathrm{k}},
$$

onde $\mathbf{R}_{\mathrm{j}}^{\mathrm{k}}$ é a $j$-ésima coluna da submatriz $\mathbf{R}^{\mathrm{k}}$, referente ao arco $e_{\mathrm{j}}$ não-básico para o produto $\mathrm{k} \mathrm{e} \mathbf{T}_{\mathbf{j}}{ }^{\mathrm{k}}$ é a $j$-ésima coluna correspondente ao mesmo arco na submatriz $\mathbf{T}^{\mathrm{k}}$.

Lembrando que $\left(\mathbf{B}^{\mathrm{k}}\right)^{-1} \mathbf{R}_{\mathrm{j}}^{\mathrm{k}}=\mathbf{y}$ (com $m$ componentes) corresponde a sequência de orientação, do único caminho na árvore básica do produto $k$, que liga o nó $F(j)$ ao nó $T(j)$. Logo, $-\left(B^{k}\right)^{-1} \mathbf{R}_{j}{ }^{k}$ corresponde a sequência de orientação dos arcos no único caminho na árvore básica que liga o nó $\mathrm{T}(\mathrm{j})$ ao nó $\mathrm{F}(\mathrm{j})$. $\quad \mathbf{P}^{\mathrm{k}}(q \times m)$ é uma matriz cujas colunas são vetores unitários ou vetores nulos, que identificam os arcos que estão na base individual $\left(\mathbf{B}^{\mathrm{k}}\right)$ do produto $\mathrm{k}$ e estão saturados.

Portanto, a operação $-\mathbf{P}^{\mathrm{k}}\left(\mathbf{B}^{\mathrm{k}}\right)^{-1} \mathbf{R}_{\mathrm{j}}^{\mathrm{k}}$, simplesmente contrai um vetor com $m$ componentes para um vetor com $q$ componentes, identificando os arcos do caminho, que estão no ciclo formado na árvore básica do produto $\mathrm{k}$ e que estão saturados, ordenando-os da maneira em que aparecem nas restrições de capacidade mútua. 
A coluna $\mathbf{T}_{\mathrm{j}}{ }^{\mathrm{k}}(q \times 1)$ da submatriz $\mathbf{T}^{\mathrm{k}}$ é um vetor unitário se o arco $e_{\mathbf{j}}$ correspondente está saturado, ou é um vetor nulo caso contrário. Portanto, para $\mathrm{i}=1, \ldots, q$, as componentes de $\mathbf{T}_{\mathbf{j}}{ }^{\mathrm{k}}-\mathbf{P}^{\mathrm{k}}\left(\mathbf{B}^{\mathrm{k}}\right)^{-1} \mathbf{R}_{\mathrm{j}}^{\mathrm{k}}$ são dadas por:

$$
\begin{aligned}
& +1 \text { se o i-ésimo arco saturado é o arco associado a } \\
\left(\mathbf{T}_{\mathrm{j}}{ }^{\mathrm{k}}-\mathbf{P}^{\mathrm{k}}\left(\mathbf{B}^{\mathrm{k}}\right)^{-} \mathbf{R}_{\mathrm{j}}\right)_{\mathrm{i}}= & +1 \text { se o i-ésimo arco saturado está no ciclo formado } \\
& \text { na árvore básica pela adição de } \mathbf{R}_{\mathrm{j}}^{\mathrm{k}} \text { (isto é, o arco } e_{\mathrm{j}} \text { ) e } \\
& \text { tem a mesma orientação que este arco; } \\
& -1 \text { se o i-ésimo arco saturado está no ciclo formado } \\
& \text { na árvore básica pela adição de } \mathbf{R}_{\mathrm{j}}^{\mathrm{k}} \text { e tem orientação } \\
& \text { contrária que o arco } e_{\mathrm{j}} ; \\
& 0 \text { caso contrário. }
\end{aligned}
$$

\subsubsection{Cálculo das variáveis duais}

A matriz básica $\left(\mathbf{B}^{*}\right)$ admite a mesma forma que a matriz $\mathbf{E}$ da seção (2.4.1) onde:
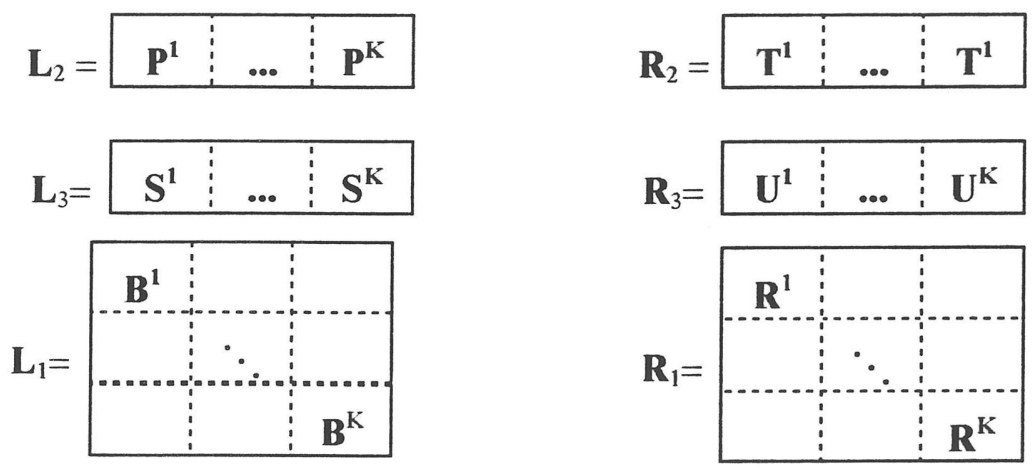

$\mathbf{L}_{1}$ é inversível e sua inversa é dada por:

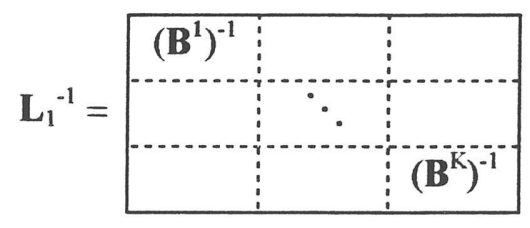

Utilizando a proposição 2.3 , veremos como a estrutura da base $\mathbf{B}^{*}$ pode ser usada eficientemente para calcular as variáveis duais, onde $\pi \mathbf{B}^{*}=\mathbf{c}$ é dado por:

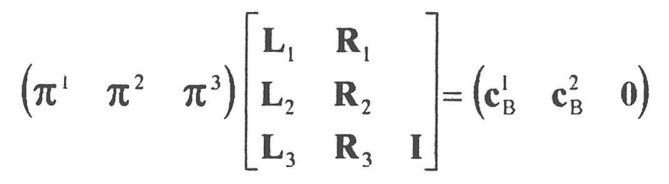

Particionando $\mathbf{c}_{\mathrm{B}}{ }^{1}$ e $\pi^{1}$ compatível com $\mathbf{L}_{1}$, particionando $\mathbf{c}_{\mathrm{B}}{ }^{2}$ e $\pi^{2}$ compatível com $\mathbf{L}_{2}$ e aplicando a proposição 2.3 onde $\pi^{3}=\mathbf{0}$, obtemos: 


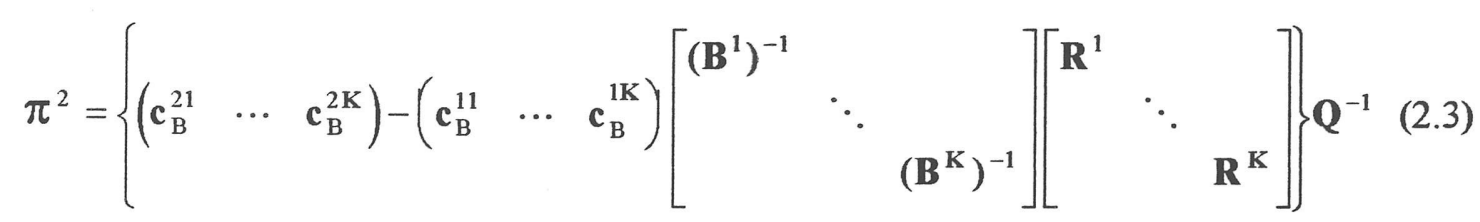

$$
\begin{aligned}
& \pi^{2}=\left[\mathbf{c}_{\mathrm{B}}{ }^{21}-\mathbf{c}_{\mathrm{B}}{ }^{11}\left(\mathbf{B}^{1}\right)^{-1} \mathbf{R}^{1} \ldots \mathrm{c}_{\mathrm{B}}{ }^{2 \mathrm{~K}}-\mathbf{c}_{\mathrm{B}}{ }^{1 \mathrm{~K}}\left(\mathbf{B}^{\mathrm{K}}\right)^{-1} \mathbf{R}^{\mathrm{K}}\right] \mathbf{Q}^{-1}
\end{aligned}
$$

Lembrando que $\left(\mathbf{B}^{\mathrm{k}}\right)^{-1} \mathbf{R}^{\mathrm{k}}$ pode ser determinado pela sequência de orientação, como na seção (1.7.5). Portanto, $\mathbf{c}_{\mathrm{B}}{ }^{2 \mathrm{k}}-\mathbf{c}_{\mathrm{B}}{ }^{1 \mathrm{k}}\left(\mathbf{B}^{\mathrm{k}}\right)^{-1} \mathbf{R}^{\mathrm{k}}$, não exige multiplicação de matrizes, apenas uma operação entre os custos. Logo, $\pi^{2}$ pode ser obtido pós-multiplicando por $\mathbf{Q}^{-1}$.

$$
\begin{aligned}
& \left.\pi^{1}=\left(\begin{array}{lll}
\pi^{11} & \ldots & \pi^{1 \mathbf{K}}
\end{array}\right)=\left\{\begin{array}{lll}
\left(\mathbf{c}_{\mathrm{B}}^{11}\right. & \cdots & \mathbf{c}_{\mathrm{B}}^{1 \mathbf{K}}
\end{array}\right)-\pi^{2}\left(\begin{array}{lll}
\mathbf{P}^{1} & \ldots & \mathbf{P}^{\mathbf{K}}
\end{array}\right)\right\}\left[\begin{array}{llll}
\left(\mathbf{B}^{1}\right)^{-1} & & \\
& \ddots & \\
& & \left(\mathbf{B}^{\mathbf{K}}\right)^{-1}
\end{array}\right] \\
& \pi^{1}=\left\{\begin{array}{l}
\pi^{11}=\left(\mathbf{c}_{\mathbf{B}}^{11}-\pi^{21} \mathbf{P}^{1}\right)\left(\mathbf{B}^{1}\right)^{-1} \\
\pi^{1 \mathbf{K}}=\left(\mathbf{c}_{\mathbf{B}}^{1 \mathbf{K}}-\pi^{2 \mathbf{K}} \mathbf{P}^{\mathbf{K}}\right)\left(\mathbf{B}^{\mathbf{K}}\right)^{-1}
\end{array}\right\} \Rightarrow\left\{\begin{array}{l}
\pi^{11} \mathbf{B}^{1}=\mathbf{c}_{\mathbf{B}}^{11}-\pi^{21} \mathbf{P}^{1} \\
\pi^{1 \mathbf{K}} \mathbf{B}^{\mathbf{K}}=\mathbf{c}_{\mathbf{B}}^{1 \mathbf{K}}-\pi^{2 \mathbf{K}} \mathbf{P}^{\mathbf{K}}
\end{array}\right\}
\end{aligned}
$$

Fazendo $\beta^{\mathrm{k}}=\mathbf{c}_{\mathrm{B}}^{1 \mathrm{k}}-\pi^{2 \mathrm{k}} \mathbf{P}^{\mathrm{k}}$ para $\mathrm{k}=1, \ldots, \mathrm{K}$ tem-se: $\pi^{1 \mathrm{k}} \mathbf{B}^{\mathrm{k}}=\beta^{\mathrm{k}}$.

Como $\mathbf{P}^{\mathrm{k}}$ tem no máximo uma entrada não-nula igual a 1 (em cada coluna), $\pi^{2 \mathrm{k}} \mathbf{P}^{\mathrm{k}}$ pode ser gerado a partir dos dados originais. Portanto, o algoritmo (1.7.2) pode ser usado para resolver o sistema acima, onde $\beta^{k}$ faz o papel dos custos das variáveis básicas.

\subsubsection{Cálculo da direção de perturbação para as variáveis básicas}

A estrutura da base $\mathbf{B}^{*}$ pode ser usada para calcular a direção de perturbação das variáveis básicas que é dada como sendo $\mathbf{y}=\left(\mathbf{B}^{*}\right)^{-1} \mathbf{N}^{j}$, onde $\mathbf{N}^{j}$ é uma coluna não-básica.

Particionando a coluna $\mathbf{N}^{\mathrm{j}}$ apropriadamente, encontrar $\mathbf{y}=\left(\mathbf{B}^{*}\right)^{-1} \mathbf{N}^{\mathrm{j}}$ é equivalente a resolver o sistema $\mathbf{B}^{*} \mathbf{y}=\mathbf{N}^{\mathrm{j}}$ dado por:

$$
\left[\begin{array}{lll}
\mathbf{L}_{1} & \mathbf{R}_{1} & \\
\mathbf{L}_{2} & \mathbf{R}_{2} & \\
\mathbf{L}_{3} & \mathbf{R}_{3} & \mathbf{I}
\end{array}\right]\left[\begin{array}{l}
\mathbf{y}^{1} \\
\mathbf{y}^{2} \\
\mathbf{y}^{3}
\end{array}\right]=\left[\begin{array}{l}
\boldsymbol{\alpha} \\
\boldsymbol{\beta} \\
\boldsymbol{\gamma}
\end{array}\right]
$$

Particionando $\mathbf{y}^{1}, \mathbf{y}^{2}, \alpha$ e $\beta$ e aplicando a proposição 2.2 , tem-se:

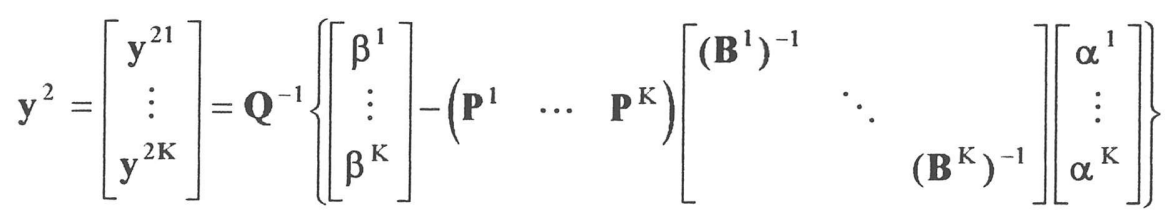

$$
\begin{aligned}
& \therefore \mathbf{y}^{2}=\mathbf{Q}^{-1}\left[\begin{array}{c}
\beta^{1}-\mathbf{P}^{1}\left(\mathbf{B}^{1}\right)^{-1} \alpha^{1} \\
\vdots \\
\beta^{K}-\mathbf{P}^{K}\left(\mathbf{B}^{K}\right)^{-1} \alpha^{K}
\end{array}\right]
\end{aligned}
$$




$$
\begin{aligned}
& \mathbf{y}^{1}=\left[\begin{array}{c}
\mathbf{y}^{11} \\
\vdots \\
\mathbf{y}^{1 \mathrm{~K}}
\end{array}\right]=\left[\begin{array}{lll}
\left(\mathbf{B}^{1}\right)^{-1} & & \\
& \ddots & \\
& & \left(\mathbf{B}^{\mathrm{K}}\right)^{-1}
\end{array}\right]\left[\begin{array}{c}
\alpha^{1} \\
\vdots \\
\alpha^{\mathrm{K}}
\end{array}\right]-\left[\begin{array}{lll}
\left(\mathbf{B}^{1}\right)^{-1} & & \\
& \ddots & \\
& & \left(\mathbf{B}^{\mathrm{K}}\right)^{-1}
\end{array}\right]\left[\begin{array}{lll}
\mathbf{R}^{1} & & \\
& \ddots & \\
& & \mathbf{R}^{\mathrm{K}}
\end{array}\right]\left[\begin{array}{c}
\mathbf{y}^{21} \\
\vdots \\
\mathbf{y}^{2 \mathrm{~K}}
\end{array}\right] \\
& \therefore \mathbf{y}^{1}=\left[\begin{array}{c}
\left(B^{1}\right)^{-1} \alpha^{1}-\left(B^{1}\right)^{-1} \mathbf{R}^{1} \mathbf{y}^{21} \\
\vdots \\
\left(\mathbf{B}^{\mathrm{K}}\right)^{-1} \alpha^{\mathrm{K}}-\left(\mathbf{B}^{\mathrm{K}}\right)^{-1} \mathbf{R}^{\mathrm{K}} \mathbf{y}^{2 \mathrm{~K}}
\end{array}\right] \\
& \mathbf{y}^{3}=\gamma-\left(\mathbf{S}^{1} \cdots \mathbf{S}^{\mathrm{K}}\right)\left[\begin{array}{c}
\left(\mathbf{B}^{1}\right)^{-1} \boldsymbol{\alpha}^{1} \\
\vdots \\
\left(\mathbf{B}^{\mathrm{K}}\right)^{-1} \boldsymbol{\alpha}^{\mathrm{K}}
\end{array}\right]-\left\{\left(\mathbf{U}^{1} \cdots \mathbf{U}^{\mathrm{K}}\right)-\left(\mathbf{S}^{1} \cdots \mathbf{S}^{\mathrm{K}}\right)\left[\begin{array}{lll}
\left(\mathbf{B}^{1}\right)^{-1} & & \\
& \ddots & \\
& & \left(\mathbf{B}^{\mathrm{K}}\right)^{-1}
\end{array}\right]\left[\begin{array}{lll}
\mathbf{R}^{1} & & \\
& \ddots & \\
& & \mathbf{R}^{\mathrm{K}}
\end{array}\right]\right\}\left[\begin{array}{l}
\mathbf{y}^{21} \\
\vdots \\
\mathbf{y}^{2 \mathrm{~K}}
\end{array}\right] \\
& \mathbf{y}^{3}=\gamma-\mathbf{S}^{1} \mathbf{y}^{11}-\ldots S^{K} \mathbf{y}^{1 \mathrm{~K}}-\mathbf{U}^{1} \mathbf{y}^{21}-\ldots-\mathbf{U}^{\mathrm{K}} \mathbf{y}^{2 \mathrm{~K}}
\end{aligned}
$$

Novamente, pode-se usar a sequência de orientação para gerar $\left(\mathbf{B}^{\mathrm{k}}\right)^{-1} \alpha^{\mathrm{k}}$ em $(2.5)$ e o mesmo acontece com $\left(\mathbf{B}^{\mathrm{k}}\right)^{-1} \mathbf{R}^{\mathrm{k}}$ em (2.6) para todo $\mathrm{k}$. Observe que somente no cálculo $\mathrm{y}^{2}$ é necessário realizar uma operação com a inversa da matriz $\mathbf{Q}$. Depois de calculado $\mathbf{y}^{2}, \mathbf{y}^{1}$ é obtido rapidamente a partir das árvores básicas $\boldsymbol{T}^{\mathrm{k}}$ de cada produto $\mathrm{k}$. Além disso, como $\mathbf{S}^{\mathrm{k}}$ e $\mathbf{U}^{\mathrm{k}}$ tem no máximo uma entrada não-nula em cada coluna, o cálculo de $y^{3}$ é simples e também não requer operações com matrizes.

\subsubsection{Algoritmo simplex especializado para o Problema de Multifluxo}

\section{Inicialização}

Seja $\left[\mathbf{x}^{\mathrm{B}}, \mathbf{x}^{\mathrm{N}}\right]$ uma solução básica factível associada a base $\mathbf{B}$. Arcos raizes e artificiais podem ser acrescentados em $\mathbf{A} \mathbf{x}^{\mathrm{k}}=\mathbf{b}^{\mathrm{k}}$ e variáveis de folga podem ser acrescentadas nas restrições de capacidade mútua para obter uma solução inicial factível.

\section{Verificação da otimalidade}

Para as variáveis não-básicas faça

$$
\begin{aligned}
& \Psi_{1}=\left\{j ; x_{j}=0 \text { e } c_{j}-\mathbf{c}^{\mathbf{B}}\left(\mathbf{B}^{*}\right)^{-1} \mathbf{N}^{\mathrm{j}}<0\right\} \\
& \Psi_{2}=\left\{\mathrm{j} ; \mathrm{x}_{\mathrm{j}}=\mathrm{u}_{\mathrm{j}} \text { e } \mathrm{c}_{\mathrm{j}}-\mathbf{c}^{\mathbf{B}}\left(\mathbf{B}^{*}\right)^{-1} \mathbf{N}^{\mathrm{j}}<0\right\}
\end{aligned}
$$

Se $\Psi_{1} \cup \Psi_{2}=\varnothing$, termine com $\left[\mathbf{x}^{\mathrm{B}}, \mathbf{x}^{\mathrm{N}}\right]$ ótimo

Senão selecione $t \in \Psi_{1} \cup \Psi_{2}$.

Faça $\delta=\left\{\begin{array}{c}1 \text { se } t \in \psi_{1} \\ -1 \text { se } t \in \psi_{2}\end{array}\right.$.

O custo reduzido $\left(\mathrm{c}_{\mathrm{j}}-\mathbf{c}^{\mathbf{B}}\left(\mathbf{B}^{*}\right)^{-1} \mathbf{N}^{j}\right)$ é calculado encontrando primeiramente o valor de $\pi$, que está particionado em $\left[\pi^{11}, \ldots, \pi^{1 \mathrm{~K}}, \pi^{2}, \pi^{3}\right]$, onde $\pi^{3}=\mathbf{0}$. Use a equação (2.3) para calcular $\pi^{2} \mathrm{e}$ (2.4) para calcular $\left[\pi^{11}, \ldots, \pi^{1 \mathrm{~K}}\right]$. 


\section{Determinação de $\Delta$ :}

Calcule $\mathbf{y}=\left(\mathbf{B}^{*}\right)^{-1} \mathbf{N}^{\mathrm{j}}$ que está particionado em $\mathbf{y}=\left[\mathbf{y}^{1}, \mathbf{y}^{2}, \mathbf{y}^{3}\right]$. Use (2.5) para encontrar o valor de $\mathbf{y}^{2}=\left[\mathbf{y}^{21}, \ldots, \mathbf{y}^{2 \mathrm{~K}}\right],(2.6)$ para obter $\mathbf{y}^{1}=\left[\mathbf{y}^{11}, \ldots, \mathbf{y}^{1 \mathrm{~K}}\right]$ e (2.7) para obter $\mathbf{y}^{3}$.

Para as variáveis básicas calcule:

$$
\Delta_{1}=\min _{\sigma\left(y_{j}\right)=\delta}\left\{\frac{x_{j}}{\left|y_{j}\right|}\right\} ; \quad \Delta_{2}=\min _{\sigma\left(y_{j}\right)=-\delta}\left\{\frac{u_{j}-x_{j}}{\left|y_{j}\right|}\right\} \text { e } \Delta=\min \left\{\Delta_{1}, \Delta_{2}, u_{j}\right\} .
$$

3. Atualização da solução

Faça $x_{j}=x_{j}+\Delta \delta$ e $x_{j}=x_{j}-\Delta \delta y_{j}$

Se $\Delta=u_{j}$ retorne ao passo 1 .

\section{Atualização da base}

$$
\text { Faça } \begin{aligned}
\psi_{3} & =\left\{\mathrm{j} ; \sigma\left(\mathrm{y}_{\mathrm{j}}\right)=\delta \text { e } \mathrm{x}_{\mathrm{j}}=0\right\} \text { e } \\
\psi_{4} & =\left\{\mathrm{j} ; \sigma\left(\mathrm{y}_{\mathrm{j}}\right)=-\delta \text { e } \mathrm{x}_{\mathrm{j}}=\mathrm{u}_{\mathrm{j}}\right\}
\end{aligned}
$$

Selecione $s \in \psi_{3} \cup \psi_{4}$ e troque $\mathbf{B}^{s}$ com $\mathbf{N}^{\mathrm{j}}$ e retorne ao passo 1 .

O método de Particionamento primal é uma especialização do método "Generalized Upper Bounding" para problemas com estruturas bloco angulares. Este método por sua vez, é uma especialização do método simplex com a inversa da base na forma produto.

A especialização do método "Generalized Upper Bounding" para problemas com estruturas bloco angulares possui uma técnica especial, desenvolvida para explorar a estrutura bloco angular da matriz de restrições e conseqüentemente da matriz básica, permitindo que o método simplex com inversa na forma produto seja aplicado, entretanto, sendo necessário manipular e armazenar somente a inversa de uma matriz de dimensão menor do que a inversa da matriz básica.

Observe que as principais operações executadas pelo método de particionamento primal requer a inversa da matriz ciclo $\left(\mathbf{Q}^{-1}\right)$ e das matrizes básicas para cada produto. Portanto, para um bom desempenho do algoritmo é necessário que se tenha disponível uma boa fórmula para atualizar a inversa da matriz ciclo.

\subsubsection{Atualização da Inversa da Matriz Ciclo}

[Hartman/Lasdon-72] mostraram que $\mathbf{Q}^{-1}$ pode ser atualizada executando no máximo duas operações pivot sobre $\mathbf{Q}^{-1}$. A fórmula dada a seguir para atualizar $\mathbf{Q}^{-1}$, pode ser encontrada no trabalho de [Helgason/kennington-8C].

A matriz básica $\mathbf{B}^{*}$ para o problema 2.2 é triangularizada pós-multiplicando-a por uma matriz de transformação denotada por $\mathbf{T}$. Seja $\mathbf{B}_{\mathrm{i}}{ }^{*}$ e $\mathbf{T}_{\mathrm{i}}$ a matriz básica e a matriz de transformação, respectivamente para a iteração $i$. Portanto, a matriz básica triangularizada na $i$-ésima iteração é dada por: 


$$
\begin{gathered}
\mathrm{B}_{\mathrm{i}}=\mathbf{B}_{\mathrm{i}}{ }^{*} \mathbf{T}_{\mathrm{i}} \\
\text { então, }\left(\mathbf{B}_{\mathrm{i}}{ }^{*}\right)^{-1}=\mathbf{T}_{\mathrm{i}}\left(\mathrm{B}_{\mathrm{i}}\right)^{-1}
\end{gathered}
$$

Semelhantemente,

$$
\begin{aligned}
& B_{i+1}=B^{*}{ }_{i+1} T_{i+1} \\
& \left(B_{i+1}\right)^{-1}=\left(T_{i+1}\right)^{-1}\left(B^{*}{ }_{i+1}\right)^{-1} .
\end{aligned}
$$

No passo 4 do algoritmo de particionamento primal, deve-se trocar a coluna básica $\mathbf{B}^{\mathrm{s}}$ pela coluna não-básica $\mathbf{N}^{\mathbf{j}}$. Portanto, a matriz básica $\mathbf{B}^{*}{ }^{*}+1$ da iteração $i+1$ difere da matriz básica da iteração anterior em apenas uma coluna. Logo,

$$
\mathbf{B}^{*}{ }_{\mathrm{i}+1}=\mathbf{E}\left(\mathbf{B}_{\mathrm{i}}^{*}\right)^{-1}
$$

onde $\mathbf{E}$ é uma matriz coluna elementar.

Entretanto, para manter a propriedade de particionamento se a coluna $\mathbf{B}^{\mathrm{s}}$ deixando a base é uma coluna chave, isto é, uma das $\mathrm{K} m$ colunas de $\mathbf{B}^{*}$, é necessário trocar esta coluna chave básica por uma outra coluna básica não-chave, antes de efetuar a mudança de base propriamente dita. Neste caso, a matriz $\mathbf{E}$ na equação (2.12) é uma matriz de permutação.

Substituindo (2.12) em (2.11) obtem-se:

$$
\left(B_{i+1}\right)^{-1}=\left(T_{i+1}\right)^{-1} \mathbf{E}\left(B_{i}^{*}\right)^{-1} .
$$

Substituindo (2.9) na equação acima, obtem-se:

$$
\left(B_{i+1}\right)^{-1}=\left(T_{i+1}\right)^{-1} \mathbf{E} \mathbf{T}_{i}\left(B_{i}\right)^{-1}
$$

Lembrando que a matriz $B=\mathbf{B} \mathbf{T}$ é dada por:

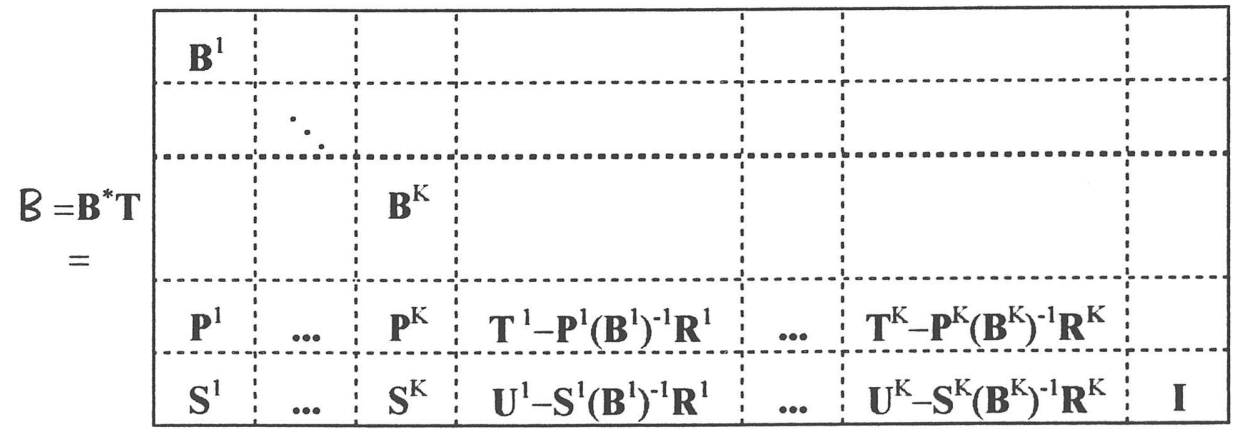

Fazendo

$$
\mathbf{Y}=\begin{array}{|c:c:c:c}
\hline \mathbf{T}^{1}-\mathbf{P}^{1}\left(\mathbf{B}^{1}\right)^{-1} \mathbf{R}^{1} & \ldots & \mathbf{T}^{\mathrm{K}}-\mathbf{P}^{\mathrm{K}}\left(\mathbf{B}^{\mathrm{K}}\right)^{-1} \mathbf{R}^{\mathrm{K}} & \\
\hdashline \mathbf{U}^{1}-\mathbf{S}^{1}\left(\mathbf{B}^{1}\right)^{-1} \mathbf{R}^{1} & \ldots & \mathbf{U}^{\mathrm{K}}-\mathbf{S}^{\mathrm{K}}\left(\mathbf{B}^{\mathrm{K}}\right)^{-1} \mathbf{R}^{\mathrm{K}} & \mathbf{I} \\
\hline
\end{array}
$$

As matrizes na equação (2.14) podem ser particionadas como:

$$
\left.\left(\mathrm{B}_{\mathrm{i}+1}\right)^{-1}=\begin{array}{|c:c}
\hline \overline{\mathbf{B}}_{\mathrm{i}+1} & \\
\hdashline \mathbf{C}_{\mathrm{i}+1} & \mathbf{Y}_{\mathrm{i}+1}^{-1} \\
\hline \mathrm{K} m & n
\end{array}\right\} \mathrm{K} m
$$

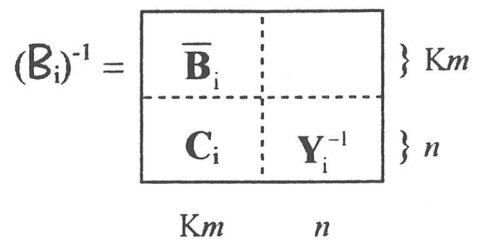

$$
\left.\mathbf{T}_{\mathbf{i}}=\begin{array}{|l|c|}
\hline \mathbf{I} & \mathbf{V}_{\mathbf{i}} \\
\hdashline & \mathbf{I} \\
\hline \mathrm{K} m & n
\end{array}\right\} \mathrm{K} m
$$




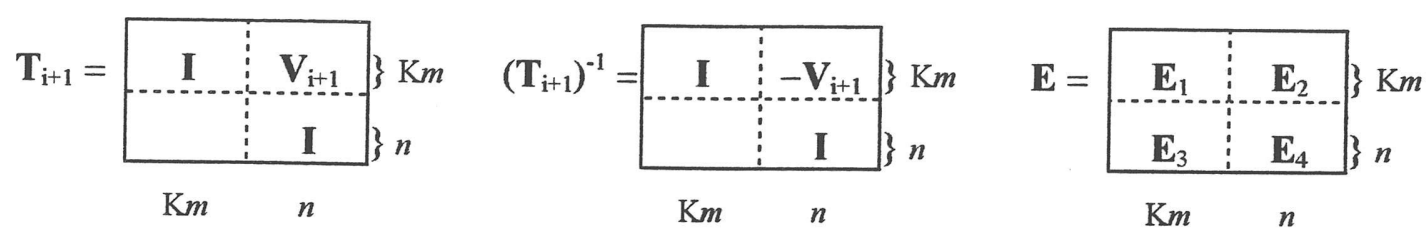
obtem-se:

Usando as matrizes particionadas acima na equação (2.14) e fazendo a multiplicação direta,

$$
\mathbf{Y}_{\mathrm{i}+1}^{-1}=\left(\mathbf{E}_{4}+\mathbf{E}_{3} \mathbf{V}_{\mathrm{i}}\right) \mathbf{Y}_{\mathrm{i}}^{-1}
$$

Note que, Y dado em (C.9) também pode ser particionada da seguinte forma:

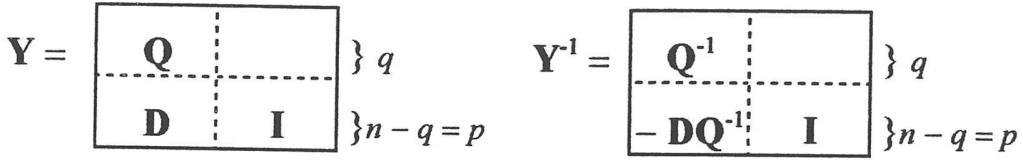

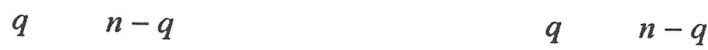

[Helgason/Kennington-80] apresentam as fórmulas de atualização de $\mathbf{Q}^{-1}$, analisando os seguintes casos:

$1^{\circ}$ Caso : A coluna saindo é uma coluna não-chave. Neste caso, a matriz $\mathbf{E}$ anterior tem a seguinte forma:

$$
\mathbf{E}=\begin{array}{|c:c}
\hline \mathbf{E}_{1} & \mathbf{E}_{2} \\
\hdashline \mathbf{E}_{3} & \mathbf{E}_{4} \\
\hline \mathbf{I} & \mathbf{E}_{2} \\
\hdashline & \mathbf{E}_{4} \\
\hline
\end{array}
$$

Como $\mathbf{E}_{3}$ é uma matriz nula, a partir de (2.17) tem-se:

$$
\mathbf{Y}_{i+1}^{-1}=\mathbf{E}_{4} \mathbf{Y}_{i}^{-1}
$$

onde $\mathbf{E}_{4}$ é uma matriz coluna elementar.

Uma coluna da matriz básica B é chamada coluna fluxo se esta coluna pertence as $q$-primeiras colunas não-chave desta base ou se ela é uma coluna não-básica entrando e diferente de uma coluna de folga. Uma linha em (2.19) é dita ser ativa se esta linha está na partição de Y correspondente a matriz $\mathbf{Q}$ e é dita ser inativa caso contrário.

Considere agora os seguintes subcasos:

Subcaso la: Ambas as colunas (a entrando e a saindo) são colunas fluxo. Particionando $\mathbf{E}_{4}$ para ser compatível com $\mathbf{Y}_{\mathrm{i}}^{-1}$ como em (2.18), tem-se:

$$
\mathbf{E}_{4}=\begin{array}{|c:c|}
\hline \mathbf{E}_{5} & \\
\hdashline \mathbf{E}_{6} & 1 \\
\hline
\end{array}
$$

Usando (2.18) e (2.20) em (2.19) obtem-se:

$$
\left.\mathbf{Y}_{\mathrm{i}+1}^{-1}=\begin{array}{|c:c}
\mathbf{Q}_{\mathrm{i}+1}^{-1} & \\
\hdashline-\mathbf{D}_{\mathrm{i}+1} \mathbf{Q}_{\mathrm{i}+1}^{-1} & \mathbf{I}
\end{array}\right]=\begin{array}{|c:c}
\mathbf{E}_{5} & \\
\hdashline \mathbf{E}_{\mathbf{6}} & \mathbf{I} \\
\hline-\mathbf{D}_{\mathbf{i}} \mathbf{Q}_{\mathrm{i}}^{-1} & \mathbf{I} \\
\hline
\end{array}
$$


Portanto, $\mathbf{Q}_{\mathbf{i}+1}^{-1}=\mathbf{E}_{5} \mathbf{Q}^{-1}$

Subcaso 1b: A coluna entrando é uma coluna fluxo e a coluna saindo é uma coluna de folga. Neste caso, antes de executar a troca de colunas, a linha correspondente a variável saindo deve tornar-se ativa.

Suponha que a variável saindo é $s_{\mathrm{n}}$. Então,

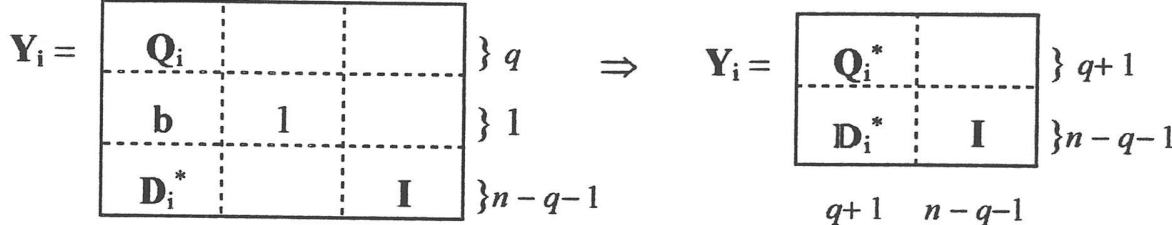

$$
\begin{aligned}
& q \quad 1 \quad n-q-1
\end{aligned}
$$

onde a $(\mathrm{q}+1)$-ésima linha de $\mathbf{Q}_{\mathrm{i}}{ }^{*}$ corresponde ao arco $e_{\mathrm{n}}$. Portanto,

$$
\left(\mathbf{Y}_{\mathrm{i}}\right)^{-1}=\begin{array}{|c:c}
\hline\left(\mathbf{Q}_{\mathrm{i}}^{*}\right)^{-1} & \\
\hdashline-\mathbf{D}_{\mathrm{i}}^{*}\left(\mathbf{Q}_{\mathrm{i}}^{*}\right)^{-1} & \mathbf{I} \\
\hline
\end{array}
$$

Note que,

$$
\left(\mathbf{Q}_{\mathrm{i}}^{*}\right)^{-1}=\begin{array}{|c:c|}
\hline\left(\mathbf{Q}_{\mathrm{i}}\right)^{-1} & \\
\hdashline-\mathrm{b}\left(\mathbf{Q}_{\mathrm{i}}\right)^{-1} & 1 \\
\hline
\end{array}
$$

Particionando $\mathbf{E}_{4}$ de modo compatível com $\mathbf{Y}_{\mathbf{i}}$ como em (2.22), obtem-se:

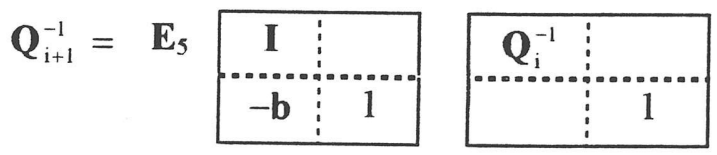

Subcaso 1c: A coluna entrando é uma coluna de folga e a coluna saindo é uma coluna fluxo.

Primeiramente é feita a mudança na inversa da matriz ciclo, a seguir a linha correspondente a variável de folga entrando deve tronar-se inativa.

A partir de (2.19) tem-se:

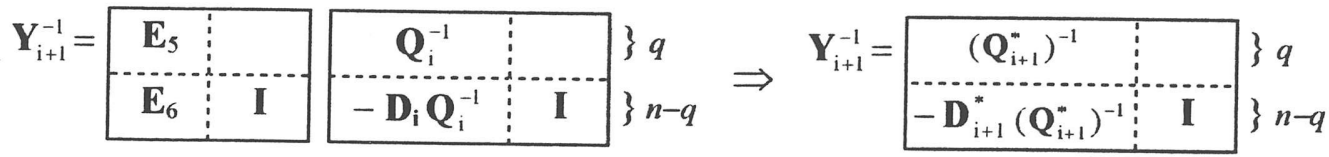

onde $\left(\mathbf{Q}_{\mathrm{i}+1}^{*}\right)^{-1}=\mathbf{E}_{5} \mathbf{Q}_{\mathrm{i}}^{-1}$

Suponha que $\mathrm{s}_{\mathrm{m}}$ é a variável de folga entrando. Suponha também que a linha ativa correspondente ao arco $e_{\mathrm{m}}$ é a $r$-ésima coluna de $\left(\mathbf{Q}_{\mathrm{i}+1}^{*}\right)^{-1}$ e a coluna saindo corresponde a $l$-ésima coluna de $\mathbf{Q}_{\mathbf{i}}$ e portantc está associada a l-ésima linha de $\left(\mathbf{Q}_{\mathbf{i}+1}^{*}\right)^{-1}$.

Portanto, $\left(\mathbf{Q}_{\mathbf{i}+1}\right)^{-1}=D_{1}\left(\mathbf{Q}_{\mathbf{i}+1}^{*}\right)^{-1} T_{\mathrm{r}}$

A matriz $D_{1}(q-1 \times q)$ é chamada matriz cancela linha, formada pela matriz identidade deletando a $l$ - linha e $T_{\mathrm{r}}$ é uma matriz $(q \times q-1)$ chamada matriz cancela coluna que também é obtida a partir da matriz identidade deletando a $r$-coluna. 
Assim, substituindo (2.26) em (2.27) obtem-se:

$$
\left(\mathbf{Q}_{i+1}\right)^{-1}=D_{1} \mathbf{E}_{5} \mathbf{Q}_{\mathrm{i}}^{-1} \boldsymbol{T}_{\mathbf{r}}
$$

Subcaso 1d: Ambas as colunas (entrando e saindo) são colunas de folga. Neste caso, antes de efetuar a mudança na inversa da matriz ciclo, a linha correspondente a variável saindo deve se tornar ativa. Em seguida é feita a mudança na matriz ciclo em seguida a linha correspondente a variável de folga entrando deve se tornar-se inativa.

Suponha que a variável saindo é $\mathrm{s}_{\mathbf{n}}$. Então,

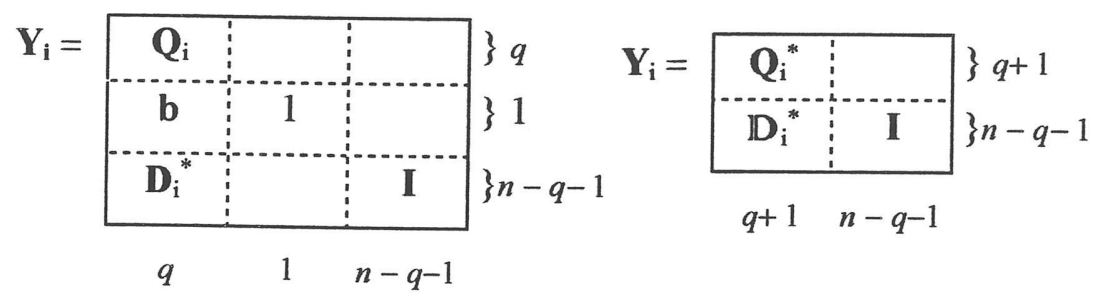

onde a ( $q+1)$-ésima linha de $\mathbf{Q}_{\mathrm{i}}{ }^{*}$ corresponde ao $\operatorname{arco} n$.

Então, como no subcaso $1 \mathrm{~b}$, obtem-se: $\left(\mathbf{Q}_{\mathbf{i}+1}^{*}\right)^{-1}=\mathbf{E}_{5}\left(\mathbf{Q}_{i}^{*}\right)^{-1}$

Suponha que a variável entrando seja $s_{\mathrm{m}}$ e que a linha ativa correspondente ao arco $\mathrm{m}$ aparece na r-ésima linha de $\mathbf{Q}_{\mathrm{i}}$.

Logo, como no subcaso 1c, tem-se:

$$
\left(\mathbf{Q}_{\mathrm{i}+1}\right)^{-1}=\boldsymbol{D}_{\mathrm{q}+1}\left(\mathbf{Q}_{\mathrm{i}+1}^{-}\right)^{-1} \boldsymbol{T}_{\mathrm{r}}
$$

Substituindo (C.23) em (C.24) obtem-se:

Portanto,

$$
\left(\mathbf{Q}_{\mathrm{i}+1}\right)^{-1}=\boldsymbol{D}_{\mathrm{q}+1} \mathbf{E}_{5}\left(\mathbf{Q}_{\mathrm{i}}^{*}\right)^{-1} T_{\mathrm{r}} .
$$

$$
\left.\mathbf{Q}_{\mathbf{i}+1}^{-1}=\boldsymbol{D}_{\mathbf{q}+1} \quad \mathbf{E}_{5} \begin{array}{|c:c}
\mathbf{I} & \\
\hdashline-\mathbf{b} & 1
\end{array}\right] \begin{array}{c|c}
\hline \mathbf{Q}_{\mathrm{i}}^{-1} & \\
\hdashline & T_{\mathrm{r}} \\
& 1 \\
\hline
\end{array}
$$

$2^{\circ}$ Caso: A coluna saindo é uma coluna chave. Então, o arco correspondente a essa coluna está em uma das árvores básicas $7_{\mathrm{b}}{ }^{\mathrm{k}}$ para algum produto $\mathrm{k}$. Se este arco é removido da base, a estrutura desta árvore básica será destruída. Portanto, antes da mudança de base propriamente dita, deve-se trocar esta coluna de $\mathbf{B}^{\mathbf{k}}$ em $\mathbf{B}^{*}$ por uma coluna de $\mathbf{R}^{\mathbf{k}}$.

Suponha que a coluna saindo está associada a $l$-ésima posição em $\mathbf{B}^{\mathrm{k}}$. Seja $\mathbf{R}_{\mathbf{j}}^{\mathrm{k}}$ a j-ésima coluna de $\mathbf{R}^{\mathrm{k}}$ escolhida para trocar de posição com $\mathbf{B}_{1}^{\mathrm{k}}$. Então, $\mathbf{R}_{\mathbf{j}}^{\mathrm{k}}$ pode substituir a $l$-ésima coluna de $\mathbf{B}^{\mathrm{k}}$, se e somente se a matriz resultante é não-singular, ou seja, $\mathrm{y}_{1} \neq 0$, onde $\mathbf{y}=\left(\mathbf{B}^{\mathrm{k}}\right)^{-1} \mathbf{R}^{\mathrm{k}}$. Considere $\beta_{1}$ a l-ésima linha de $\left(\mathbf{B}^{\mathrm{k}}\right)^{-1}$, logo $y_{1}=\beta_{1} \mathbf{R}_{\mathbf{j}}^{\mathrm{k}}$. Seja $\gamma=\beta_{1} \mathbf{R}^{\mathrm{k}}$, existem dois subcasos.

Subcaso $2 a: \gamma \neq \mathbf{0}$. Suponha que $\gamma_{\mathbf{j}} \neq 0$, então a $j$-ésima coluna de $\mathbf{R}^{\mathbf{k}}$ pode trocar de posição com a $l$-ésima coluna de $\mathbf{B}^{\mathrm{k}}$ e o particionamento será preservado. 
Considere a seguinte matriz de permutação dada a seguir.

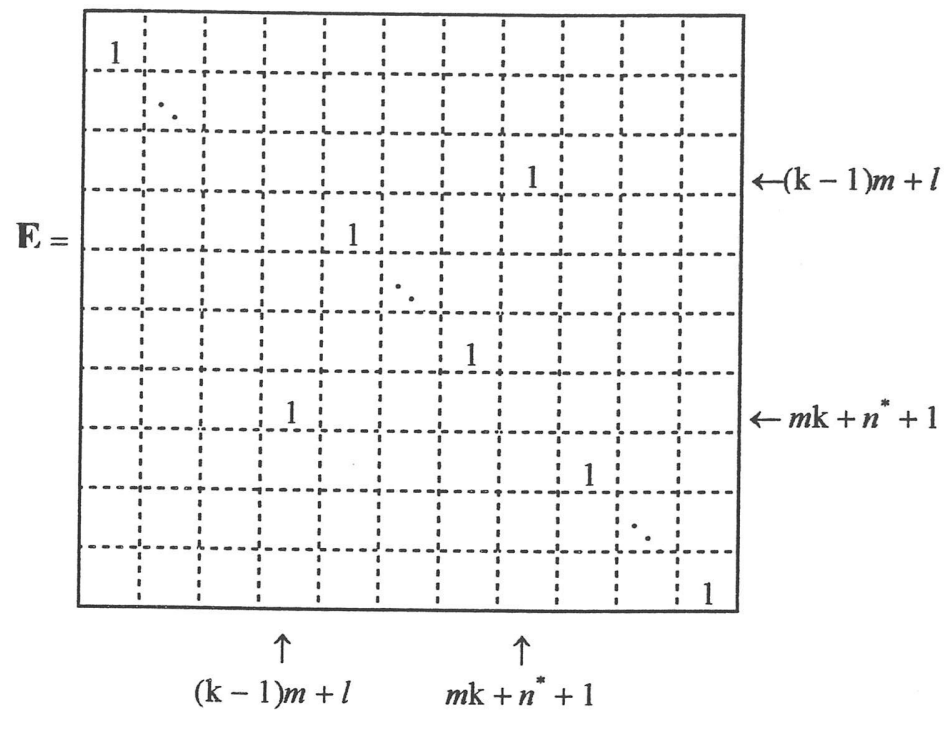

onde $n^{*}$ é dado por: $n^{*}=\left\{\begin{array}{l}0 \quad \text { se } \mathrm{k}=1 \\ \sum_{\mathrm{k}=1}^{\mathrm{K}} \mathrm{v}_{\mathrm{k}} \text { caso contrario }\end{array}\right.$ e $\mathrm{v}_{\mathrm{k}}$ é o número de colunas de cada bloco da matriz $\mathbf{V}_{\mathrm{i}}$ definida como:

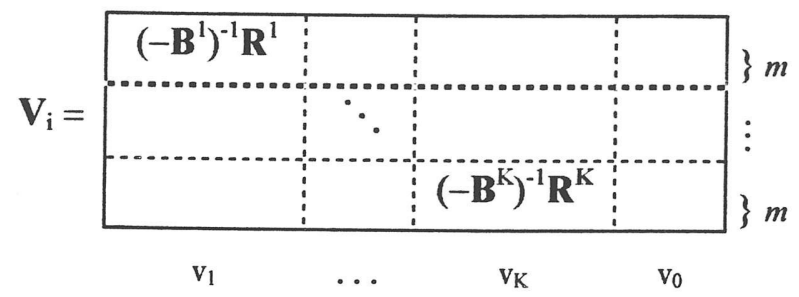

Então, $\mathbf{B}^{*}{ }_{i+1}$ (a matriz formada pela matriz $\mathbf{B}_{\mathrm{i}}{ }^{*}$ trocando a coluna $(\mathrm{k}-1) m+l$ com a coluna $\left.m \mathrm{k}+n^{*}+1\right)$ é dada por $\mathbf{B}_{\mathrm{i}}{ }^{*} \mathbf{E}$. Então, $\mathbf{B}^{*}{ }^{*}+1=\mathbf{E}^{-1}\left(\mathbf{B}_{\mathrm{i}}{ }^{*}\right)^{-1}$, mas $\mathbf{E}^{-1}=\mathbf{E}$.

Logo, $\quad \mathbf{B}^{*}{ }_{i+1}=\mathbf{E}\left(\mathbf{B}_{\mathrm{i}}^{*}\right)^{-1}$.

A matriz $\mathbf{E}$ está particionada de maneira compatível com $\mathbf{B}_{i}{ }^{*}$, onde,
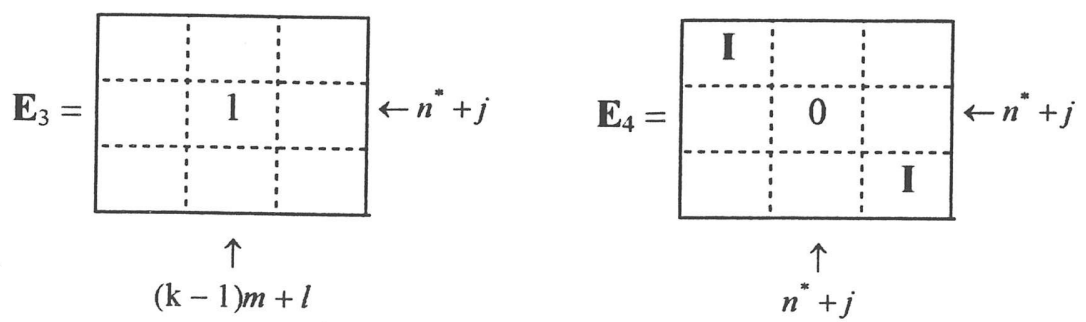

Assim, $\mathbf{E}_{4}+\mathbf{E}_{3} \mathbf{V}_{\mathrm{i}}$ da equação (2.17) resulta na matriz linha elementar da forma:

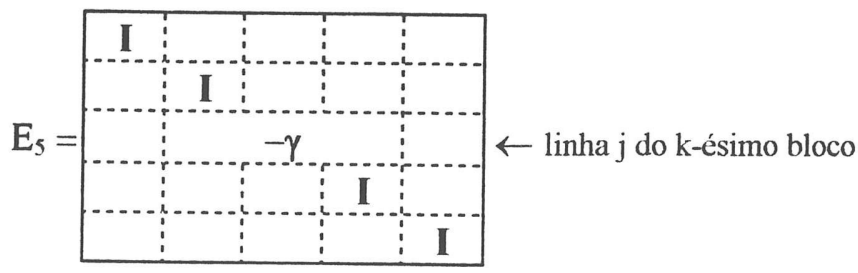


Portanto, $\mathbf{Y}_{\mathrm{i}+1}^{-1}=\mathbf{E}_{5} \mathbf{Y}_{\mathrm{i}}^{-1}$.

Particionando $\mathbf{E}_{5}$ de forma compatível com (2.18), tem-se:

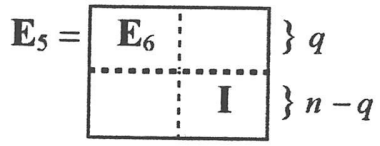

Substituindo (2.37) e (2.18) em (2.34) obtem-se:

\begin{tabular}{|c|c|}
\hline $\mathbf{Q}_{\mathrm{i}+1}^{-1}$ & \\
\hdashline$-\mathbf{D}_{\mathbf{i}} \mathbf{Q}_{\mathrm{i}+1}^{-1}$ & $\mathbf{I}$ \\
\hline
\end{tabular}$=$\begin{tabular}{|c:c|c|}
\hline $\mathbf{E}_{\mathbf{6}}$ & \\
\hdashline & $\mathbf{I}$ \\
\hdashline$-\mathbf{D}_{\mathbf{i}} \mathbf{Q}_{\mathrm{i}}^{-1}$ & $\mathbf{I}$ \\
\hline
\end{tabular}

Portanto, $\quad \mathbf{Q}_{i+1}^{-1}=\mathbf{E}_{6} \mathbf{Q}_{\mathrm{i}}^{-1}$

Subcaso 2b: $\gamma=\mathbf{0}$. Então, ambas as colunas (entrando e saindo) devem estar associadas com o produto k e uma troca direta é possível. Caso contrário, o particionamento de $\mathbf{B}^{*}$ poderia não ser possível. Portanto, não existe mudança na matriz ciclo. 


\section{Capítulo 3}

\section{A Decomposição de}

\section{Problemas de Multifluxo em Redes}

\subsection{Introdução}

A decomposição é utilizada como ferramenta para o desenvolvimento de métodos para resolver problemas que possuam estruturas especiais, como por exemplo, o problema de multifluxo em redes, cuja matriz de restrições tem a forma ilustrada na figura 2.1.

Quando as técnicas de decomposição são utilizadas em um problema com essa estrutura, obtem-se um problema de otimização chamado problema mestre, que está relacionado ao conjunto de restrições consideradas complicadas, e um conjunto de subproblemas correspondentes ao conjunto de restrições simples. As soluções dos subproblemas embora sejam independentes, são coordenadas pelo problema mestre de modo que produzam uma solução para o problema original.

Uma motivação para a utilização da abordagem de decomposição para problemas de multifluxo em redes tendo $\mathrm{K}$ produtos, é que estes possuem $\mathrm{K}$ subconjuntos de restrições de conservação de fluxo (um para cada produto), para os quais existem algoritmos eficientes, e um conjunto formado pelas restrições de capacidade mútua que impedem a resolução do problema como problemas de fluxo em rede independentes.

Neste capítulo apresentaremos duas técnicas de decomposição, a decomposição recursodiretor e decomposição preço-diretor. A decomposição recurso-diretor consiste em distribuir a capacidade mútua dos arcos decompondo o problema de multifluxo em rede em $\mathrm{K}$ problemas de fluxo em rede de custo mínimo coordenados por um problema mestre. Neste trabalho esta abordagem será resolvida uma especialização do método Otimização Subgradiente de [Held et al-74] proposta por [Kennington/Shalaby-77].

A técnica de decomposição preço-diretor para resolver problemas de multifuxo em redes que será apresentada neste trabalho, foi proposta por [Dantzig/Wolfe -61] e é conhecida como Decomposição Dantzig-Wolfe. No capítulo seguinte mostramos o trabalho de Jones et al. (1993) que estuda o impacto desta decomposição em diferentes formulações para o problema de multifluxo. 


\subsection{A Decomposição recurso-diretor}

A decomposição recurso-diretor, quando aplicada para decompor problemas de multifluxo em redes, consiste em distribuir a capacidade mútua dos arcos, fixando temporariamente esta capacidade sobre o fluxo nos arcos para cada um dos produtos. Portanto, para um problema de multifluxo tendo $\mathrm{K}$ produtos, quando a capacidade é temporariamente fixada, o problema de multifluxo é decomposto em $\mathrm{K}$ subproblemas de fluxo em rede de custo mínimo (um para cada produto) os quais são chamados de subproblemas.

Entretanto, a distribuição da capacidade mútua dos arcos não deve ser feita de forma aleatória, mas de forma que as soluções dos subproblemas tenham uma solução factível e que estas soluções produzam uma solução factível e mínima para o problema original.

Considere o problema de multifluxo dado por:

$$
\begin{array}{ll}
\text { Minimizar } & \sum_{\mathrm{k}=1}^{\mathrm{K}} \mathbf{c}^{\mathrm{k}} \mathbf{x}^{\mathrm{k}} \\
\text { sujeito a } & \mathbf{A \mathbf { x } ^ { \mathrm { k } }}=\mathbf{b}^{\mathrm{k}} \quad \mathrm{k}=1, \ldots, \mathrm{K} \\
& \sum_{\mathrm{k}=1}^{\mathrm{K}} \mathbf{D}^{\mathrm{k}} \mathbf{x}^{\mathrm{k}} \leq \mathbf{r} \\
& \mathbf{0} \leq \mathbf{x}^{\mathrm{k}} \leq \mathbf{u}^{\mathrm{k}} \quad \mathrm{k}=1, \ldots, \mathrm{K}
\end{array}
$$

onde A é a matriz incidência nó-arco $(m \times n)$.

Seja $\mathbf{y}=\left(\mathbf{y}^{1}, \mathbf{y}^{2}, \ldots, \mathbf{y}^{\mathrm{K}}\right)$ uma distribuição do vetor de capacidade mútua $\mathbf{r}$, sobre os arcos para cada produto $\mathrm{k}(\mathrm{k}=1, \ldots, \mathrm{K})$. Ou seja, $\mathrm{y}^{\mathrm{k}}$ é um vetor com valores temporariamente fixados sobre as capacidades mútuas dos arcos na rede para o produto $\mathrm{k}$.

Portanto, o problema de multifluxo (3.1) está separado em K subproblemas de fluxo em rede de custo mínimo, um para cada produto, da forma:

$$
\begin{array}{ll}
\text { Minimizar } & \mathbf{c}^{\mathrm{k}} \mathbf{x}^{\mathrm{k}} \\
\text { sujeito a } & \mathbf{A} \mathbf{x}^{\mathrm{k}}=\mathbf{b}^{\mathrm{k}} \\
& \mathbf{0} \leq \mathbf{x}^{\mathrm{k}} \leq \mathbf{y}^{\mathrm{k}} \quad \forall \mathrm{k}
\end{array}
$$

Estes problemas são problemas de fluxo em rede de custo mínimo e podem ser resolvidos pelo método simplex para redes apresentado no capítulo 1 .

Considere $Z^{k}\left(y^{k}\right)$ o valor ótimo para o subproblema $k$ quando a distribuição é $\mathbf{y}^{\mathrm{k}}(\mathrm{k}=1, \ldots, \mathrm{K})$, isto é, $Z^{\mathrm{k}}\left(\mathbf{y}^{\mathrm{k}}\right)=\min \left\{\mathbf{c}^{\mathrm{k}} \mathbf{x}^{\mathbf{k}} ; \mathbf{A} \mathbf{x}^{\mathbf{k}}=\mathbf{b}^{\mathrm{k}}\right.$ e $\left.\mathbf{0} \leq \mathbf{x}^{\mathrm{k}} \leq \mathbf{y}^{\mathrm{k}}\right\}$.

Portanto, para que as soluções obtidas pelos subproblemas (3.2) sejam factíveis para o problema de multifluxo (3.1), a soma das capacidades distribuídas sobre cada arco (para todos produtos), deve ser menor ou igual a capacidade mútua deste arco como no problema original. Ou seja, deve satisfazer as seguintes condições: 


$$
\begin{aligned}
& \text { (i) } \sum_{\mathrm{k}=1}^{\mathrm{K}} \mathbf{D}^{\mathrm{k}} \mathbf{y}^{\mathrm{k}} \leq \mathbf{r} \\
& \text { (ii) } \mathbf{0} \leq \mathbf{y}^{\mathrm{k}} \leq \mathbf{u}^{\mathrm{k}} \text { para } \mathrm{k}=1, \ldots, \mathrm{K} \text {. }
\end{aligned}
$$

Para que as soluções ótimas (mínimas) dos subproblemas (3.2) sejam também ótimas para o problema de multifluxo (3.1), a distribuição das capacidades mútuas deve ser feita de modo a minimizar a soma dos valores das função dos subproblemas, isto é, minimizar a soma de $Z^{\mathrm{k}}\left(\mathbf{y}^{\mathrm{k}}\right)$, $\mathrm{k}=1, \ldots, \mathrm{K}$.

Portanto, o problema (3.1) é equivalente ao seguinte problema:

$$
\begin{gathered}
\text { Minimizar } Z\left(\mathbf{y}^{1}, \mathbf{y}^{2}, \ldots, \mathbf{y}^{\mathrm{K}}\right)=\sum_{\mathbf{k}=1}^{\mathrm{K}} \mathbf{Z}^{\mathrm{k}}\left(\mathbf{y}^{\mathrm{k}}\right) \\
\text { sujeito a } \quad \sum_{\mathrm{k}=1}^{\mathrm{K}} \mathbf{D}^{\mathrm{k}} \mathbf{y}^{\mathrm{k}} \leq \mathbf{r} \\
\quad \mathbf{0} \leq \mathbf{y}^{\mathrm{k}} \leq \mathbf{u}^{\mathrm{k}} \quad \mathrm{k}=1, \ldots, \mathrm{K} .
\end{gathered}
$$

onde $Z^{k}\left(\mathbf{y}^{k}\right)=\min \left\{\mathbf{c}^{\mathrm{k}} \mathbf{x}^{\mathrm{k}} ; \mathbf{A} \mathbf{x}^{\mathrm{k}}=\mathbf{b}^{\mathrm{k}}\right.$ e $\left.\mathbf{0} \leq \mathbf{x}^{\mathrm{k}} \leq \mathbf{y}^{\mathrm{k}}\right\}$.

O problema (3.4) é chamado problema mestre. Este problema coordena as distribuições e as soluções ótimas dos subproblemas, para que estas forneçam um solução factível e ótima para o problema de multifluxo (3.1).

[Kennington-78] comenta que J.T. Robacker em 1956, foi quem primeiro propôs esta abordagem de solução para resolver problemas de multifluxo em redes, mas não especificou como esta abordagem poderia ser resolvida.

Proposição 3.1: A função $Z(y)=Z\left(y^{1}, y^{2}, \ldots, y^{K}\right)$ é convexa sobre o conjunto $\left\{y^{1}, y^{2}, \ldots, y^{K}\right.$; $\left.\mathbf{y}^{1} \geq \mathbf{0}, \mathbf{y}^{2} \geq \mathbf{0}, \ldots, \mathbf{y}^{\mathrm{K}} \geq \mathbf{0}\right\}$.

Como o problema (3.4) requer a minimização de uma função convexa sobre um conjunto convexo e compacto, se a função $Z(y)$ fosse sempre diferenciável, condições de otimalidade convenientes poderiam ser estabelecidas e um algoritmo que produz uma sequência de soluções factíveis, isto é, um algoritmo de direções factíveis poderia ser aplicado. Entretanto, $Z(y)$ nem sempre é diferenciável (linear por partes). Portanto, algoritmos deste tipo não podem ser aplicados diretamente.

\subsubsection{Resolvendo o problema mestre pelo método Otimização do Subgradiente}

[Held et al - 74] propuseram um método baseado no método do Subgradiente de N.Z. Shor, chamado Otimização do Subgradiente e o aplicaram para resolver o problema mestre da distribuição (3.4) para problemas de fluxo máximo de multiprodutos o qual foi adaptado por [Kennington/Shalaby -77] para resolver problemas de multifluxo de custo mínimo . 
Subgradiente: Um vetor $\eta$ é um subgradiente de uma função convexa $Z$ em $\mathbf{y}^{\prime}$ se:

$$
Z(\mathbf{y})-Z\left(y^{\prime}\right) \geq \eta\left(\mathbf{y}-\mathbf{y}^{\prime}\right), \forall \mathbf{y} \in \operatorname{dom} Z \text {. }
$$

$\mathrm{O}$ conjunto de todos os subgradientes de $Z$ em $\mathbf{y}^{\prime}$ é denotado por $\partial Z\left(\mathbf{y}^{\prime}\right)$ e é chamado de subdiferencial.

No trabalho de [Held et al - 74], uma distribuição inicial factível, era dada como:

$$
\sum_{k=1}^{\mathrm{K}} \mathbf{D}^{\mathrm{k}} \mathbf{y}^{\mathrm{k}}=\mathbf{r}
$$

onde os arcos não possuíam restrições de capacidade individual, um subgradiente era utilizado para definir a direção de movimento.

A motivação para a escolha do subgradiente de $Z$ em uma distribuição y como direção de movimento é dada pela proposição a seguir.

Proposição 3.2: [Geoffrion-70] Seja $\mathbf{x}^{\mathrm{k}^{*}}$ uma solução ótima para $Z^{\mathrm{k}}\left(\overline{\mathbf{y}}^{\mathrm{k}}\right)$. Então, $\mathbf{v}^{\mathrm{k}^{*}}$ é o vetor multiplicador Lagrangeano ótimo associado a restrição $\mathrm{x}^{\mathrm{k}} \leq \overline{\mathbf{y}}^{\mathrm{k}}$ de $\mathrm{Z}^{\mathrm{k}}\left(\overline{\mathbf{y}}^{\mathrm{k}}\right)$ se e somente se $-v^{\mathrm{k}^{*}}$ é um subgradiente de $Z^{\mathrm{k}}$ em $\overline{\mathbf{y}}^{\mathrm{k}}$.

Proposição 3.3: A função $Z^{\mathrm{k}}\left(\mathrm{y}^{\mathrm{k}}\right)$ é diferenciável se e somente se o dual tem solução ótima única.

Se a função objetivo é diferenciável no ponto de interesse, então o único subgradiente coincide com o gradiente da função e esta direção produz uma solução melhor para o problema. No entanto, mesmo quando este não é o caso, um passo é feito na direção estabelecida.

Portanto, a função objetivo pode aumentar, diminuir ou permanecer a mesma para iterações sucessivas.

Seja - v um subgradiente de $Z$ no ponto $\mathbf{y}^{\prime}$. A nova distribuição obtida quando $\mathbf{y}^{\prime}$ é perturbado na direção $-v$ é dada por:

$$
\overline{\mathbf{y}}=\mathbf{y}^{\prime}+\theta(-v), \text { onde } \theta \text { é o passo. }
$$

[Held et al - 74] comenta que B.T. Poljak em 1967 mostrou um resultado geral sobre a escolha apropriada do passo $\theta$ para o método do Subgradiente de N.Z. Shor. Este resultado afirma que o passo $\theta$ deve ser escolhido de modo que satisfaça a Regra das Séries Divergentes, isto é, a sequência de passos $\theta_{\mathrm{i}}$ deve satisfazer as seguintes condições:

$$
\text { (i) } \theta_{\mathrm{i}} \rightarrow 0 \text { e (ii) } \sum_{\mathrm{i}=0}^{\infty} \theta_{\mathrm{i}}=\infty \text {. }
$$

Trabalhando com problemas de maximização, B.T.Poljak em 1969, propôs um passo definido da seguinte forma:

$$
\theta_{i}=\lambda_{i} \frac{Z^{*}-Z\left(y_{i}\right)}{\|\eta\|^{2}},
$$


onde $\eta$ é um subgradiente e $\lambda$ é uma sequência não-crescente onde $0 \leq \lambda \leq 2, Z^{*}$ é o valor ótimo da função objetivo e $Z\left(y_{i}\right)$ é o valor da função para a solução corrente. Como o valor ótimo $Z^{*}$ é o valor procurado, B.T.Poljak mostrou que usando uma estimativa $\hat{Z}$ inferior ao valor ótimo $\left(\hat{Z}<Z^{*}\right)$ e $\lambda=2$, a sequência $Z\left(y_{i}\right)$ ou converge para $\hat{Z}$ ou obtem-se, em um número finito de iterações, um ponto $\mathrm{y}_{\mathrm{L}}$ para o qual, $\hat{\mathrm{Z}} \leq \mathrm{Z}\left(\mathrm{y}_{\mathrm{L}}\right) \leq \mathrm{Z}^{*}$.

Considere $\mathbf{y}^{*}$ como qualquer ponto no conjunto de soluções ótimas para $Z$, isto é, $Z\left(y^{*}\right)=Z^{*}$, a idéia principal para a utilização deste passo é que: como $\eta \in \partial Z\left(y_{i}\right)$, pela definição de subgradiente tem-se $Z^{*}-Z\left(y_{i}\right) \geq \eta\left(y^{*}-y_{i}\right)$. Desta maneira, se $y_{i}$ não é ótima, então a direção $\eta$ faz um ângulo agudo com o raio a partir de $\mathbf{y}_{\mathbf{i}}$ passando por $\mathbf{y}^{*}$. Consequentemente, se o tamanho do passo $\theta_{\mathbf{i}}$ é suficientemente pequeno, o ponto $\mathbf{y}_{\mathbf{i}+1}$ pode estar mais próximo de $\mathbf{y}^{*}$ do que $\mathbf{y}_{\mathbf{i}}$. Portanto, a sequência de valores que se aproxima do conjunto ótimo.

[Held et al - 74] utilizaram em suas experiências computacionais uma estimativa superior $\bar{Z}$ para problemas de maximização, isto é, $\bar{Z}>Z^{*}$. Pois, a estimativa $\hat{Z}$ inferior poderia ser dificil de encontrar. Observando os resultados, afirmaram que a convergência não depende estritamente sobre o valor exato da estimativa, sendo sobretudo necessário que a condição $\theta_{i} \rightarrow 0$ seja satisfeita. Então, como a estimativa para o valor ótimo era superior, sugeriram uma regra para escolher a sequência $\left\{\lambda_{i}\right\}$ de forma que $\lambda_{i} \rightarrow 0$. Geralmente, uma boa regra é fazer $\lambda_{i}=2$ durante 2D iterações (onde D é a dimensão do problema), a seguir reduz-se sucessivamente, o valor de $\lambda$ e do número de iterações à metade, até que o número de iterações atinja algum número $\mathrm{L}$ pré-fixado. Então, $\lambda$ é reduzido à metade a cada $L$ iterações, até que $\theta_{i}$ seja suficientemente pequeno.

[Kennington/Shalaby -77] utilizaram para problemas de multifluxo em redes de custo mínimo, uma estimativa inferior $\bar{Z}\left(\bar{Z}<Z^{*}\right)$. Portanto, o passo $\theta$ era dado por:

$$
\theta_{i}=\lambda_{i} \frac{Z\left(y_{i}\right)-\bar{Z}}{\|\eta\|^{2}}
$$

Como o tamanho do passo é selecionado baseado somente no comportamento da funçạ̃o, a nova distribuição y geralmente é infactível. Então, [Held et al - 74] propôs uma operação chamada Operação Projeção, para fazer com que a nova distribuição se torne factível, onde a nova solução é dada por: $\mathbf{y}=P[\overline{\mathbf{y}}+\theta(-\boldsymbol{v})]$, onde $P[$.$] projeta a solução sobre o conjunto$

$$
\mathrm{Y}=\left\{\mathbf{y}=\left(\mathbf{y}^{1}, \mathbf{y}^{2}, \ldots, \mathbf{y}^{\mathrm{K}}\right) ; \sum_{\mathrm{k}=1}^{\mathrm{K}} \mathbf{D}^{\mathrm{k}} \mathbf{y}^{\mathrm{k}}=\mathbf{r}, \mathbf{0} \leq \mathbf{y}^{\mathrm{k}} \leq \mathbf{u}^{\mathrm{k}} \mathrm{k}=1, \ldots, \mathrm{K}\right\} .
$$

$\mathrm{O}$ algoritmo termina quando o subgradiente no ponto de interesse é único, isto é, $\eta=\mathbf{0} \in$ $\partial Z\left(y^{\prime}\right)$. Como este critério de parada não é computacionalmente eficiente, [Kennington/Shalaby -77] fizeram uma adaptação para o método terminar quando a solução obtida esteja próxima da solução 
ótima quanto se queira, isto é, escolher um $\varepsilon>0$ para o método terminar se $Z\left(y_{i}{ }^{\prime}\right)-\bar{Z}<\varepsilon(\bar{Z})$, ou quando um número máximo de iterações L pré-fixado fosse alcançado. [Helgason et al-79] apresentaram uma caracterização das soluções para problemas quadráticos com uma única restrição, que pode ser utilizada, eficientemente, para realizar a operação projeção que será descrita posteriormente.

[Helgason/Kennington-80] usaram o método proposto por [Kennington/Shalaby-77] para resolver problemas de multifluxo de custo mínimo, onde os arcos possuíam capacidade individual e utilizando a caracterização da solução para problemas quadráticos de [Helgason et al-79] para fazer a projeção da solução perturbada na direção oposta do subgradiente.

Considere o problema de multifluxo dado por 3.1. Acrescentando as variáveis artificiais (vetor $\mathbf{a}^{\mathbf{k}}$ ) e as variáveis de folga (vetor $\mathbf{s}$ ), obtem-se:

$$
\begin{aligned}
& \operatorname{Minimizar} \sum_{\mathrm{k}=1}^{\mathrm{K}} \mathbf{c}^{\mathrm{k}} \mathbf{x}^{\mathrm{k}}+\mathbf{M} \mathbf{1} \mathbf{a}^{\mathrm{k}} \\
& \text { sujeito a } \quad \mathbf{A x}^{\mathrm{k}}+\mathbf{a}^{\mathbf{k}}=\mathbf{b}^{\mathbf{k}} \quad \mathbf{k}=1, \ldots, \mathbf{K} \text {. } \\
& \sum_{k=1}^{K} D^{k} x^{k}+s=r \\
& \mathbf{0} \leq \mathbf{x}^{\mathrm{k}} \leq \mathbf{u}^{\mathrm{k}} \text { e } \mathbf{a}^{\mathrm{k}} \geq \mathbf{0} \forall \mathrm{k}
\end{aligned}
$$

onde $\mathrm{M}$ é um escalar positivo muito grande.

Na primeira distribuição cada arco tem capacidade igual a $\mathbf{y}^{\mathbf{k}}=\mathbf{u}^{\mathbf{k}}$. Se algum dos subproblemas $Z^{\mathrm{k}}\left(\mathrm{y}^{\mathrm{k}}\right)$ for infactível, então o problema (3.11) será infactível consequentemente (3.1) também será infactível. Se todos os subproblemas são factíveis, então tem-se um limite inferior para a solução do problema dado a seguir.

$$
\begin{aligned}
& \text { Minimizar } Z\left(\mathbf{y}^{1}, \mathbf{y}^{2}, \ldots, \mathbf{y}^{\mathrm{K}}\right)=\sum_{\mathrm{k}=1}^{\mathrm{K}} \mathrm{Z}^{\mathrm{k}}\left(\mathbf{y}^{\mathrm{k}}\right) \\
& \text { sujeito a } \sum_{\mathrm{k}=1}^{\mathrm{K}} \mathbf{D}^{\mathrm{k}} \mathbf{y}^{\mathrm{k}}=\mathbf{r} \\
& \qquad \mathbf{0} \leq \mathbf{y}^{\mathrm{k}} \leq \mathbf{u}^{\mathrm{k}} \quad \mathrm{k}=1, \ldots, \mathrm{K} .
\end{aligned}
$$

onde $Z^{k}\left(\mathbf{y}^{k}\right)=\min \left\{\mathbf{c}^{\mathrm{k}} \mathbf{x}^{\mathrm{k}}+\mathbf{M} 1 \mathbf{a}^{\mathrm{k}} ; \mathbf{A} \mathbf{x}^{\mathrm{k}}+\mathbf{a}^{\mathrm{k}}=\mathbf{b}^{\mathrm{k}}\right.$ e $\left.\mathbf{x}^{\mathrm{k}}+\mathbf{s}^{\mathrm{k}}=\mathbf{y}^{\mathrm{k}}, \mathbf{x}^{\mathrm{k}} \geq \mathbf{0} \mathbf{a}^{\mathrm{k}} \geq \mathbf{0}\right\}$, ou pela teoria da dualidade $Z^{k}\left(\mathbf{y}^{k}\right)=\max \left\{\mathbf{b}^{k} \mu^{k}-\mathbf{y}^{k} v^{k} ; \mu^{k} \mathbf{A} v^{k} \leq \mathbf{c}^{k}, \mu^{k} \leq \mathbf{M} 1, v^{k} \geq 0\right\}$.

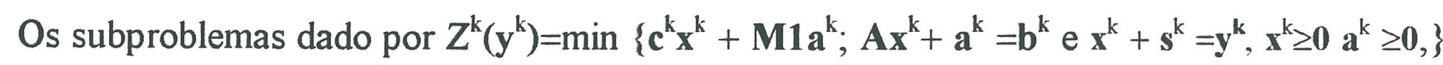
são problemas de fluxo em rede de custo mínimo e podem ser resolvidos pelo método simplex para redes apresentado no capítulo 1, usando uma transformação para remover a capacidade individual $\left(\mathrm{x}^{\mathrm{k}} \leq \mathrm{y}^{\mathrm{k}}\right)$ do problema de fluxo em rede, para obter $\left(\mathrm{v}^{\mathrm{k}}\right.$ 


\subsubsection{Algoritmo: Otimização do subgradiente para o problema de multifluxo}

\section{Inicialização}

Escolha um $\varepsilon>0$ para terminar se para algum $i$, a solução $\mathbf{y}_{\mathrm{i}}$ encontrada é tal que $\mathrm{Z}\left(\mathbf{y}_{\mathrm{i}}\right)-\mathrm{LB}<\varepsilon(\overline{\mathrm{Z}})$, onde LB é o limite inferior. Selecione um inteiro $\mathrm{L}>0$ denotando o número máximo de distribuições permitidas e a sequência de $\lambda$ 's. Faça $Z^{*}=\infty$ e $i=0$.

\section{Determinar um limite inferior}

Faça a distribuição $\mathbf{y}^{\mathrm{k}}=\mathbf{u}^{\mathrm{k}}$ para $\mathrm{k}=1, \ldots \mathrm{K}$. Então, o limite inferior é dado por $\mathrm{LB}=\sum_{\mathrm{k}=1}^{\mathrm{K}} Z^{\mathrm{k}}\left(\mathbf{y}^{\mathrm{k}}\right)$, onde $Z^{k}\left(\mathbf{y}^{k}\right)=\min \left\{\mathbf{c}^{\mathrm{k}} \mathbf{x}^{\mathrm{k}}+\mathbf{M} 1 \mathbf{a}^{\mathrm{k}} ; \mathbf{A} \mathbf{x}^{\mathrm{k}}+\mathbf{a}^{\mathrm{k}}=\mathbf{b}^{\mathrm{k}} \mathrm{e} \mathbf{x}^{\mathrm{k}}+\mathbf{s}^{\mathrm{k}}=\mathbf{y}^{\mathrm{k}}, \mathbf{x}^{\mathrm{k}} \geq \mathbf{0} \quad \mathbf{a}^{\mathrm{k}} \geq \mathbf{0}\right\}$,

Se $\mathbf{a}^{\mathrm{k}} \neq \mathbf{0}$, então o problema (3.1) não tem solução.

Caso contrário, faça $\left(\mathbf{y}_{0}{ }^{1}, \mathbf{y}_{0}{ }^{2}, \ldots, \mathbf{y}_{0}{ }^{\mathrm{K}}\right)=\mathrm{P}\left[\mathbf{x}_{\mathrm{o}}{ }^{1}, \mathbf{x}_{\mathrm{o}}{ }^{2}, \ldots, \mathbf{x}_{\mathrm{o}}{ }^{\mathrm{K}}\right]$, onde $\left(\mathrm{x}_{\mathrm{o}}{ }^{1}, \mathbf{x}_{\mathrm{o}}{ }^{2}, \ldots, \mathbf{x}_{\mathrm{o}}{ }^{\mathrm{K}}\right)$ é a solução ótima de $Z^{\mathrm{k}}\left(\mathbf{y}^{\mathrm{k}}\right) \mathrm{k}=1, \ldots, \mathrm{K} P[\cdot]$ é projeção sobre $\mathrm{Y}=\left\{\mathbf{y}=\left(\mathbf{y}^{1}, \mathbf{y}^{2}, \ldots, \mathbf{y}^{\mathrm{K}}\right) ; \sum_{\mathrm{k}=1}^{\mathrm{K}} \mathbf{D}^{\mathrm{k}} \mathbf{y}^{\mathrm{k}}=\mathbf{r}, \mathbf{0} \leq \mathbf{y}^{\mathrm{k}} \leq \mathbf{u}^{\mathrm{k}} \mathbf{k}=1, \ldots, \mathrm{K}\right\}$.

\section{Resolver $K$ subproblemas}

$Z^{k}\left(\mathbf{y}_{i}^{k}\right)=\min \left\{\mathbf{c}^{\mathrm{k}} \mathbf{x}^{\mathrm{k}}+\operatorname{M1} \mathbf{a}^{\mathrm{k}} ; \mathbf{A} \mathbf{x}^{\mathrm{k}}+\mathbf{a}^{\mathrm{k}}=\mathbf{b}^{\mathrm{k}} \mathrm{e} \mathbf{x}^{\mathrm{k}}+\mathbf{s}^{\mathrm{k}}=\mathbf{y}_{\mathrm{i}}^{\mathrm{k}}, \mathbf{x}^{\mathrm{k}} \geq \mathbf{0}, \mathbf{a}^{\mathrm{k}} \geq \mathbf{0}\right\}$

Se $\sum_{\mathrm{k}=1}^{\mathrm{K}} \mathrm{Z}^{\mathrm{k}}\left(\mathrm{y}_{\mathrm{i}}^{\mathrm{k}}\right)<\mathrm{Z}^{*}$ faça $\mathrm{Z}^{*}=\sum_{\mathrm{k}=1}^{\mathrm{K}} \mathrm{Z}^{\mathrm{k}}\left(\mathrm{y}_{\mathrm{i}}^{\mathrm{k}}\right)$, vá para o passo 3 .

Senão vá para o passo 5 .

3. Verificar a $\varepsilon$ - otimalidade

Se $Z\left(y_{i}^{k}\right)-Z^{*}<\varepsilon\left(Z^{*}\right)$ vá para o passo 4 .

Se não vá para o passo 5 .

\section{Checar a factibilidade}

Se para a solução correspondente a $Z^{*}$ todas as variáveis artificiais são nulas, termine.

Se não vá para o passo 5 .

\section{Determinação de uma nova distribuição (Projeção)}

Se $i=\mathrm{L}$, termine com a melhor solução encontrada

Caso contrário, faça $\left(\mathbf{y}_{i+1}{ }^{1}, \mathbf{y}_{\mathrm{i}+1}{ }^{2}, \ldots, \mathbf{y}_{\mathrm{i}+1}{ }^{\mathrm{K}}\right)=\mathrm{P}\left[\left(\mathbf{y}_{\mathrm{i}}{ }^{1}, \mathbf{y}_{\mathrm{i}}{ }^{2}, \ldots, \mathbf{y}_{\mathrm{i}}^{\mathrm{K}}\right)+\theta_{l}\left(-\boldsymbol{v}^{1},-\boldsymbol{v}^{2}, \ldots,-\boldsymbol{v}^{\mathrm{K}}\right)\right]$, onde $\theta_{\mathrm{i}}=\lambda_{\mathrm{i}} \frac{Z\left(\mathbf{y}_{\mathrm{i}}^{1}, \mathbf{y}_{\mathrm{i}}^{2}, \ldots, \mathbf{y}_{\mathrm{i}}^{\mathrm{k}}\right)-Z^{*}}{\sum_{\mathrm{k}} v_{\mathrm{i}}^{\mathrm{k}} v_{\mathrm{i}}^{\mathrm{k}}}$. Faça $i=i+1$ e retorne ao passo 2

\subsubsection{A Operação Projeção}

Considere $\overline{\mathbf{y}}=\mathbf{y}^{\prime}+\theta v$, a operação projeção é definida como sendo qualquer $\mathbf{y} \in \mathrm{Y}=\left\{\mathbf{y}=\left(\mathbf{y}^{1}, \mathbf{y}^{2}, \ldots, \mathbf{y}^{\mathrm{K}}\right) ; \sum_{\mathrm{k}=1}^{\mathrm{K}} \mathbf{D}^{\mathrm{k}} \mathbf{y}^{\mathrm{k}}=\mathbf{r}, \mathbf{0} \leq \mathbf{y}^{\mathbf{k}} \leq \mathbf{u}^{\mathrm{k}} \mathrm{k}=1, \ldots, \mathrm{K}\right\}$ o mais próximo de $\overline{\mathbf{y}}$ segundo a norma Euclidiana. Portanto, para realizar a operação projeção o seguinte problema deve ser resolvido.

Minimizar $\left\{\left\|\left(\mathbf{y}^{1}, \mathbf{y}^{2}, \ldots, \mathbf{y}^{\mathrm{k}}\right)-\left(\overline{\mathbf{y}}^{1}, \overline{\mathbf{y}}^{2}, \ldots, \overline{\mathbf{y}}^{\mathrm{k}}\right)\right\| ; \sum_{\mathrm{k}} \mathbf{D}^{\mathrm{k}} \mathbf{y}^{\mathrm{k}}=\mathbf{r}, \mathbf{0} \leq \mathbf{y}^{\mathrm{k}} \leq \mathbf{u}^{\mathrm{k}} \forall \mathbf{k}\right\}$ 


$$
\begin{aligned}
& \equiv \operatorname{Minimizar}\left\{\left(\sum_{j, k}\left(y_{j}^{k}-\bar{y}_{j}^{k}\right)^{2}\right)^{1 / 2} ; \sum_{k} \mathbf{D}^{\mathrm{k}} \mathbf{y}^{\mathrm{k}}=\mathbf{r}, \mathbf{0} \leq \mathbf{y}^{\mathrm{k}} \leq \mathbf{u}^{\mathrm{k}} \forall \mathrm{k}\right\} \\
& \equiv \operatorname{Minimizar}\left\{\sum_{\mathrm{j}, \mathrm{k}}\left(\mathrm{y}_{\mathrm{j}}^{\mathrm{k}}-\overline{\mathrm{y}}_{\mathrm{j}}^{\mathrm{k}}\right)^{2} ; \sum_{\mathrm{k}} \mathbf{D}^{\mathrm{k}} \mathbf{y}^{\mathrm{k}}=\mathbf{r}, \mathbf{0} \leq \mathbf{y}^{\mathrm{k}} \leq \mathbf{u}^{\mathrm{k}} \forall \mathrm{k}\right\} .
\end{aligned}
$$

Este problema pode ser decomposto em j. Portanto, para cada arco j o seguinte problema deve ser resolvido:

$$
\operatorname{Minimizar}\left\{\sum_{\mathrm{k}}\left(\mathrm{y}_{\mathrm{j}}^{\mathrm{k}}-\overline{\mathrm{y}}_{\mathrm{j}}^{\mathrm{k}}\right)^{2} ; \sum_{\mathrm{k}} \mathrm{D}_{\mathrm{j} j}^{\mathrm{k}} \mathrm{y}_{\mathrm{j}}^{\mathrm{k}}=\mathrm{r}_{\mathrm{j}}, 0 \leq \mathrm{y}_{\mathrm{j}}^{\mathrm{k}} \leq \mathrm{u}_{\mathrm{j}}^{\mathrm{k}} \forall \mathrm{k}\right\} \text {. }
$$

Por simplicidade de notação, para evitar o índice $j z_{k}=D_{j j}^{k} y_{j}^{k}$ e $c=r_{j}(3.12)$ acima pode ser apresentado de forma equivalente como:

onde $\overline{\mathbf{D}}$ é uma matriz diagonal.

$$
\begin{gathered}
\text { Minimizar } \frac{1}{2} \mathbf{z} \overline{\mathbf{D}} \mathbf{z}-\mathbf{a z} \\
\text { sujeito a } \sum_{\mathbf{k}}^{2} z_{\mathbf{k}}=\mathrm{c} \\
\mathbf{0} \leq \mathbf{z} \leq \mathbf{u},
\end{gathered}
$$

Reescrevendo as restrições (3.13) obtem-se:

$$
\begin{gathered}
\text { Minimizar } \frac{1}{2} \mathbf{z} \overline{\mathbf{D}} \mathbf{z}-\mathbf{a z} \\
\text { sujeito a } \sum_{\mathrm{k}} z_{\mathrm{k}}=\mathrm{c} \\
\mathrm{z}_{\mathrm{k}}-\mathrm{u}_{\mathrm{k}} \leq 0 \\
-\mathrm{z}_{\mathrm{k}} \leq 0
\end{gathered}
$$

onde $\lambda, w_{k}, v_{k}$ são os multiplicadores de Kuhn-Tucker associados com os três tipos de restrições.

As condições de Kuhn-Tucker para o problema (3.14) podem ser apresentadas como:

$$
\begin{aligned}
& \mathrm{d}_{\mathrm{k}} \mathrm{z}_{\mathrm{k}}-a_{\mathrm{k}}+\mathrm{w}_{\mathrm{k}}-\mathrm{v}_{\mathrm{k}}+\lambda=0 \quad \forall \mathrm{kj}, \\
& \mathrm{w}_{\mathrm{k}}\left(\mathrm{z}_{\mathrm{k}}-\mathrm{u}_{\mathrm{k}}\right)=0 \quad \forall \mathrm{k}, \\
& \mathrm{v}_{\mathrm{k}} \mathrm{z}_{\mathrm{k}}=0 \quad \forall \mathrm{k}, \\
& \mathrm{w}_{\mathrm{k}}, \mathrm{v}_{\mathrm{k}} \geq 0 \quad \forall \mathrm{k} .
\end{aligned}
$$

mais as condições (3.14b), (3.14c) e (3.14d). $d_{k}$ é o k-ésimo elemento diagonal de $\overline{\mathbf{D}}$.

Considere a seguinte solução como função de $\lambda$ :

$$
\begin{aligned}
& \mathrm{z}_{\mathrm{k}}(\lambda)=\max \left\{\min \left[\frac{a_{\mathrm{k}}-\lambda}{\mathrm{d}_{\mathrm{k}}}, \mathrm{u}_{\mathrm{k}}\right], 0\right\}, \\
& \mathrm{w}_{\mathrm{k}}(\lambda)=\max \left\{a_{\mathrm{k}}-\lambda-\mathrm{d}_{\mathrm{k}} \mathrm{u}_{\mathrm{k}}, 0\right\} \\
& \mathrm{v}_{\mathrm{k}}(\lambda)=\max \left\{\lambda-a_{\mathrm{k}}, 0\right\} .
\end{aligned}
$$

Para qualquer escolha de $\lambda$ a solução dada por (3.16) satisfaz (3.14c), (3.14d).e (3.15d).

Proposição 3.4: A solução dada por (3.16) satisfaz (3.15b), (3.15c) e (3.15a). 
Portanto, para resolver (3.16) é necessário encontrar um $\lambda$ apropriado tal que (3.14b) seja satisfeita, isto é, $\sum_{\mathbf{k}} z_{\mathbf{k}}=c$.

$$
\mathrm{g}(\lambda)=\sum_{\mathbf{k}} \mathrm{z}_{\mathrm{k}}(\lambda)=\sum_{\mathbf{k}} \max \left\{\min \left[\frac{a_{\mathrm{k}}-\lambda}{\mathrm{d}_{\mathrm{k}}}, \mathrm{u}_{\mathrm{k}}\right], 0\right\} .
$$

Então, $\lambda^{*}$ deve ser encontrado para que $g\left(\lambda^{*}\right)=c$. Como $d_{k} \geq 0$ e $u_{k} \geq 0 \quad z_{k}(\lambda)$ pode ser dada por:

$$
\mathrm{z}_{\mathrm{k}}(\lambda)= \begin{cases}\mathrm{u}_{\mathrm{k}} & \text { se } \lambda \leq a_{\mathrm{k}}-\mathrm{d}_{\mathrm{k}} \mathrm{u}_{\mathrm{k}} \\ \frac{a_{\mathrm{k}}-\lambda}{\mathrm{d}_{\mathrm{k}}} & \text { se } a_{\mathrm{k}}-\mathrm{d}_{\mathrm{k}} \mathrm{u}_{\mathrm{k}}<\lambda \leq a_{\mathrm{k}} \\ 0 & \text { se } \lambda>a_{\mathrm{k}}\end{cases}
$$

Cada $z_{k}(\lambda)$ é uma função linear por partes e monotônica não-crescente. A soma de tais funções preserva esta propriedade. Assim, $g(\lambda)$ também é uma função linear por partes e monotônica não-crescente.

Note que que $z$ tem $n$ componentes. Então, os pontos de quebra para a função linear por partes $\mathrm{g}(\lambda)$ ocorre em $2 \mathrm{~K}$ pontos: $a_{\mathrm{k}}-\mathrm{d}_{\mathrm{k}} \mathrm{u}_{\mathrm{k}}$ e $a_{\mathrm{k}}$ para $\mathrm{j}=1, \ldots, n$. Seja $\alpha_{1}, \alpha_{2}, \ldots, \alpha_{2 \mathrm{~K}}$ os $2 \mathrm{~K}$ pontos de quebra onde $\alpha_{1} \leq \alpha_{2} \leq \ldots \leq \alpha_{2 \mathrm{~K}}$.

Então, para $\lambda \leq \alpha_{1} \Rightarrow g(\lambda)=\sum_{\mathbf{k}} \mathrm{u}_{\mathbf{k}}$ e para $\lambda \geq \alpha_{2 K} \Rightarrow \mathrm{g}(\lambda)=0$. Portanto, o problema (3.13) tem solução factível se e somente se $0 \leq \mathrm{c} \leq \sum_{\mathbf{k}} \mathrm{u}_{\mathbf{k}}$. O algoritmo a seguir obtem $\lambda^{*}$ tal que $\mathrm{g}\left(\lambda^{*}\right)=\mathrm{c}$.

\section{Algoritmo para obter $\lambda^{*}$}

0. Inicialização

Se $c>\sum_{j} u_{j}$ ou $c<0$, termine problema não tem solução.

Se não faça $l=1, \mathrm{r}=2 \mathrm{~K}, \mathrm{~L}=\sum_{\mathbf{k}} \mathrm{u}_{\mathbf{k}}$ e R $=0$.

1. Teste de parada

Se $\mathrm{r}-l=1$, vá para o passo 4 .

Se não $m=[(l+\mathrm{r}) / 2]_{\mathrm{I}}$ onde $[\mathrm{k}]_{\mathrm{I}}$ : é o maior inteiro $\leq \mathrm{k}$.

2. Cálculo do novo valor

Faça $C=\sum_{\mathbf{k}} \max \left\{\min \left[\frac{a_{\mathbf{k}}-\alpha_{m}}{\mathrm{~d}_{\mathbf{k}}}, \mathrm{u}_{\mathbf{k}}\right], 0\right\}$, onde $\mathrm{C}=\sum_{\mathbf{k}} \mathrm{z}_{\mathbf{k}}(\lambda)$.

\section{Atualização}

Se $C=c$, termine com a sullição $\lambda^{*}=\alpha_{m}$.

Se C $>$ c, faça $l=m, \mathrm{~L}=\mathrm{C}$ e vá para o passo 1 .

$\mathrm{Se} \mathrm{C}<\mathrm{c}$, faça $\mathrm{r}=m, \mathrm{R}=\mathrm{C}$ e vá para o passo 1 .

\section{Interpolação}

Termine com $\lambda^{*}=\alpha_{l}+\left[\left(\alpha_{\mathrm{r}}-\alpha_{l}\right)(\mathrm{c}-\mathrm{L})\right] /(\mathrm{R}-\mathrm{L})$. 
Com o valor obtido pelo algoritmo 3.2.3, os valores de $\mathrm{z}, \mathrm{w}, \mathrm{v}$ são determinados segundo (3.16). Assim $y_{j}^{k}=z_{k} / d_{j}$ é a projeção procurada.

\subsection{A Decomposição de Dantzig-Wolfe}

O nome decomposição preço-diretor é utilizado para classificar técnicas de decomposição que coordenam a solução entre o problema mestre e os subproblemas, alterando as funções objetivo dos subproblemas através dos multiplicadores de Lagrange (variáveis duais). Os métodos que utilizam este tipo de decomposição, procuram encontrar multiplicadores apropriados, de maneira que as soluções combinadas dos subproblemas Lagrangeanos, resultantes da decomposição, seja uma solução ótima para o problema original.

[Kennington - 78] comenta que Ford e Fulkerson em 1958 foram os primeiros a sugerirem uma abordagem de decomposição preço-diretor, para resolver problemas de fluxo máximo de multiprodutos, apresentando a técnica de geração de colunas para a formulação arco-caminho.

\subsubsection{Decomposição Dantzig- Wolfe para Problemas de Multifluxo em redes}

[Dantzig/Wolfe-61] apresentaram um algoritmo de decomposição para problemas com uma matriz de restrições bloco angular e restrições adicionais. Tais problemas, geralmente, possuem um número muito grande de variáveis e restrições, portanto a base para esses problemas também possui uma dimensão muito grande. Uma maneira de lidar com essa dificuldade, sugerida por [Dantzig/Wolfe-61] é transformar o problema original em um problema equivalente com poucas restrições, escrevendo o problema de forma equivalente, em função dos pontos extremos de um subconjunto das restrições. Quando esta transformação é feita o problema equivalente possui um número de restrições menor, mas um número extremamente maior de variáveis. No entanto, utilizando o método simplex para resolver o problema equivalente, uma técnica de geração de colunas pode ser utilizada.

Uma motivação para aplicação da Decomposição de Dantzig-Wolfe para problemas de multifluxo (3.1), é que este problema possui a estrutura descrita acima, onde as restrições de conservação de fluxo $\left(\mathbf{A} \mathbf{x}^{\mathrm{k}}=\mathbf{b}^{\mathrm{k}}, \mathbf{0} \leq \mathbf{x}^{\mathrm{k}} \leq \mathbf{u}^{\mathrm{k}}\right)$, formam o conjunto de restrições bloco angulares, enquanto que as restrições de capacidade mútua constitui as restrições adicionais. Assim decompondo o problema de multifluxo, os subproblemas formados podem ser resolvidos por métodos eficientes existentes, por exemplo o método simplex apresentado no capítulo 1 .

O desenvolvimento da decomposição de Dantzig-Wolfe está baseado no fato de que, um ponto pertence a um poliedro convexo limitado se e somente se, este ponto pode ser escrito como combinação convexa dos pontos extremos deste poliedro. 
Proposição 3.5: Seja $S=\{\mathbf{x}: \mathbf{A x}=\mathbf{b}, \mathbf{x} \geq \mathbf{0}\}$ não vazio e limitado e $\mathbf{x}^{\mathrm{i}}, \mathrm{i}=1, \ldots, \mathrm{q}$, seus pontos extremos. Então, qualquer $\mathbf{x} \in S$ pode ser escrito como,

$$
x=\sum_{i=1}^{q} \lambda_{i} x^{i}, \sum_{i=1}^{q} \lambda_{i}=1 \text { e } \lambda_{i} \geq 0 i=1, \ldots, q .
$$

Considere o problema de multifluxo em rede dado a seguir, onde a rede possui $m$ nós e $n$ arcos e existem $\mathrm{K}$ produtos que percorrem a rede (um produto está definido apenas pelas características físicas dos itens que percorrem a rede, portanto, $\mathrm{K}$ itens distintos definem $\mathrm{K}$ produtos).

$$
\begin{array}{ll}
\text { Minimizar } & \sum_{\mathrm{k}=1}^{\mathrm{K}} \mathbf{c}^{\mathrm{k}} \mathbf{x}^{\mathrm{k}} \\
\text { sujeito a } & \mathbf{A \mathbf { x } ^ { \mathrm { k } }}=\mathbf{b}^{\mathrm{k}} \quad \mathrm{k}=1, \ldots, \mathrm{K} \\
& \sum_{\mathrm{k}=1}^{\mathrm{K}} \mathbf{D}^{\mathrm{k}} \mathbf{x}^{\mathrm{k}} \leq \mathbf{r} \\
& \mathbf{0} \leq \mathbf{x}^{\mathrm{k}} \leq \mathbf{u}^{\mathbf{k}} \quad \mathrm{k}=1, \ldots, \mathrm{K}
\end{array}
$$

onde A é a matriz incidência nó-arco $(m \times n)$.

As restrições $\mathbf{A} \mathbf{x}^{\mathbf{k}}=\mathbf{b}^{\mathrm{k}} \mathrm{k}=1, \ldots, \mathrm{K}$ formam um conjunto de restrições bloco angular, que pode ser visto como uma matriz Â com $\mathrm{K}$ submatrizes, dada da forma:

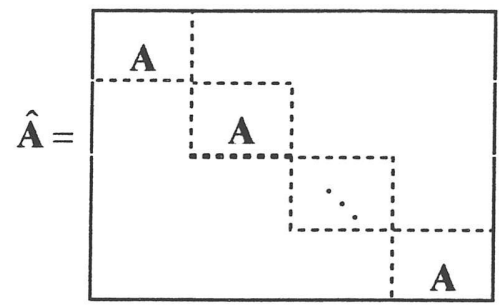

Considerando a matriz $\hat{\mathbf{A}}$ dada, as restrições (3.17b) e (3.17d) podem ser apresentadas como:

$$
\begin{aligned}
& \hat{\mathbf{A}} \mathbf{x}=\hat{\mathbf{b}} \text { onde } \mathbf{x}=\left(\mathbf{x}^{1}, \mathbf{x}^{2}, \ldots, \mathbf{x}^{\mathrm{K}}\right)^{\prime} \text { e } \hat{\mathbf{b}}=\left(\mathbf{b}^{1}, \mathbf{b}^{2}, \ldots, \mathbf{b}^{\mathrm{K}}\right)^{\prime}, \\
& \mathbf{0} \leq \mathbf{x} \leq \mathbf{u} \text { onde } \mathbf{u}=\left(\mathbf{u}^{1}, \mathbf{u}^{2}, \ldots, \mathbf{u}^{\mathrm{K}}\right)^{\prime} .
\end{aligned}
$$

Considere o conjunto $S=\left\{\mathbf{x}=\left(\mathbf{x}^{1}, \mathbf{x}^{2}, \ldots, \mathbf{x}^{\mathrm{K}}\right)^{\prime} ; \hat{\mathbf{A}} \mathbf{x}=\hat{\mathbf{b}}\right.$ e $\left.\mathbf{0} \leq \mathbf{x} \leq \mathbf{u}\right\}$. Observe que, $S$ é formado por um conjunto de restrições independentes. Portanto, um ponto extremo para $S$ é da forma $\overline{\mathbf{x}}_{\mathrm{j}}=\left(\overline{\mathbf{x}}_{\mathbf{j}}{ }^{1}, \overline{\mathbf{x}}_{\mathbf{j}}^{2}, \ldots, \overline{\mathbf{x}}_{\mathrm{j}}^{\mathrm{K}}\right)$ onde $\overline{\mathbf{x}}_{\mathrm{j}}^{\mathrm{k}}$ é um ponto extremo solução para $X^{\mathrm{k}}=\left\{\mathbf{A} \mathbf{x}^{\mathrm{k}}=\mathbf{b}^{\mathrm{k}}\right.$ e $\left.\mathbf{0} \leq \mathbf{x}^{\mathrm{k}} \leq \mathbf{u}^{\mathrm{k}}\right\}$.

Seja $\mathbf{x} \in S$, então

$$
\mathbf{x}^{0}=\sum_{j} \overline{\mathbf{x}}_{\mathrm{j}} \lambda_{\mathrm{j}} \text {, onde } \sum_{\mathrm{j}=1} \lambda_{\mathrm{j}}=1 \text { e } \lambda_{\mathrm{j}} \geq 0 .
$$
problema:

Usando a representação acima para $\mathbf{x}$, o problema (3.17) é equivalente ao seguinte 


$$
\begin{gathered}
\text { Minimizar } \sum_{j}\left(\mathbf{c} \overline{\mathbf{x}}_{\mathbf{j}}\right) \lambda_{j} \\
\text { sujeito a } \quad \sum_{\mathbf{j},} \sum_{\mathbf{k}=1}^{\mathrm{K}}\left(\mathbf{D}^{\mathrm{k}} \overline{\mathbf{x}}_{\mathbf{j}}^{\mathrm{k}}\right) \lambda_{\mathbf{j}}+\mathbf{I} \mathbf{s}=\mathbf{r} \\
\quad \sum_{\mathbf{j}} \lambda_{\mathbf{j}}=1 \\
\lambda_{\mathbf{j}} \geq 0 \text { e } \mathbf{s} \geq \mathbf{0}
\end{gathered}
$$

Definido, $\sum_{\mathrm{k}} \mathbf{D}^{\mathrm{k}} \overline{\mathbf{x}}_{\mathrm{j}}^{\mathrm{k}}=\mathbf{P}_{\mathrm{j}}$

$$
\mathbf{c} \overline{\mathbf{x}}_{\mathrm{j}}=\overline{\mathbf{c}}_{\mathrm{j}}
$$

onde $\mathbf{c}=\left(\mathbf{c}^{1} \mathbf{c}^{2} \ldots, \mathbf{c}^{\mathrm{K}}\right)$, o problema (3.20) pode ser apresentado como:

$$
\begin{array}{ll}
\text { Minimizar } & \sum_{\mathbf{j}} \overline{\mathbf{c}}_{\mathbf{j}} \lambda_{\mathbf{j}} \\
\text { sujeito a } & \sum_{\mathbf{j}} \mathbf{P}_{\mathbf{j}} \lambda_{\mathrm{j}}+\mathbf{I} \mathbf{s}=\mathbf{r} \\
& \sum_{\mathbf{j}} \lambda_{\mathrm{j}}=1 \\
& \lambda_{\mathbf{j}} \geq 0 \quad \text { e } \mathbf{s} \geq \mathbf{0}
\end{array}
$$

Este problema é chamado problema mestre, e é equivalente ao problema de multifluxo (3.17), possuindo somente $(n+1)$ restrições comparando com as $(n+\mathrm{K} m)$ restrições do problema (3.17). Entretanto, possui um número extremamente grande de variáveis, tantos quantos forem os pontos extremos para o conjunto $S$.

Proposição 3.6: Se $\left\{\lambda_{\mathrm{j}}\right\}$ resolve o problema (3.22), então os vetores

$$
\mathbf{x}^{0}=\sum_{j} \overline{\mathbf{x}}_{\mathrm{j}} \lambda_{\mathrm{j}} \quad \text { resolvem o problema (3.17). }
$$

A matriz de restrições para este problema é da forma:

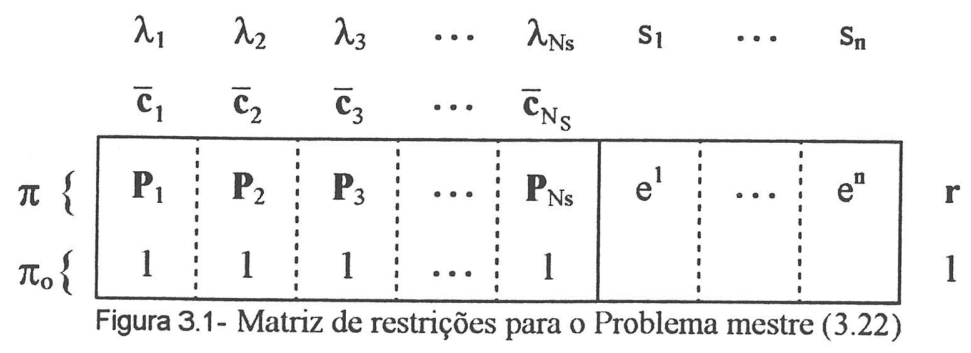

Este problema mestre possui $(n+1)$ restrições. Portanto, devem existir $(n+1)$ colunas que constituam l'ma base $\mathbf{B}$ factível. Considere $\mathbf{B}_{(n+1 \times n+1)}$ uma base factível pa a o problema (3.22) e $\overline{\mathbf{c}}^{\mathrm{B}}$ o custo das variáveis associadas a esta base. A única solução básica não-negativa associada a esta base satisfazendo (3.22b) e (3.22c), é obtida atribuindo-se valor nulo para as variáveis $\lambda_{\mathrm{j}}$ associadas as colunas restantes, ou seja, as colunas não-básicas.

Se esta base não é ótima, uma coluna não-básica deve ser selecionada para entrar em $\mathbf{B}$. 
Considere $\left(\pi, \pi_{\mathrm{o}}\right)$ o vetor de multiplicadores Lagrangeanos (variáveis duais) associadas as restrições (3.22b) e (3.22c) como mostra a figura 3.1 acima.

Utilizando o método simplex para resolver o problema mestre (3.22), um dos passos requeridos para selecionar uma coluna não-básica para entrar na base é analisar o custo reduzido das variáveis não-básicas, dado por:

(i) $\overline{\mathbf{c}}_{\mathrm{j}}^{\pi}=\overline{\mathbf{c}}_{\mathrm{j}}-\left(\pi, \pi_{\mathrm{o}}\right)\left[\begin{array}{r}\mathbf{P}_{\mathrm{j}} \\ 1\end{array}\right]$ correspondentes a variável $\lambda_{\mathrm{j}}$,

(ii) $\overline{\mathbf{c}}_{\mathbf{j}}^{\pi}=0-\left(\pi, \pi_{\mathbf{o}}\right)\left[\begin{array}{r}\mathbf{e}_{\mathbf{j}} \\ 0\end{array}\right]$ correspondentes as variáveis de folga $\mathrm{s}_{\mathbf{j}}$.

Se o custo reduzido é não-negativo, isto é,

$$
\begin{aligned}
& \text { (i) } \overline{\mathbf{c}}_{\mathrm{j}}^{\pi}=\overline{\mathbf{c}}_{\mathrm{j}}-\pi \mathbf{P}_{\mathrm{j}}-\pi_{\mathrm{o}} \geq 0 \\
& \text { (ii) } \overline{\mathbf{c}}_{\mathrm{j}}^{\pi}=0-\pi \mathrm{e}^{\mathrm{j}}=-\pi_{\mathrm{j}} \geq 0
\end{aligned}
$$

a solução corrente associada a base $\mathbf{B}$ é ótima.

Se as condições (i) e (ii) acima, não forem satisfeitas, uma coluna não-básica deverá ser selecionada para entrar na base.

Considere $\overline{\mathbf{c}}_{\mathrm{j}}^{\pi}=\overline{\mathbf{c}}_{\mathrm{j}}-\pi \mathbf{P}_{\mathrm{j}}-\pi_{\mathrm{o}} \mathrm{o}$ custo reduzido associado a variável $\lambda_{\mathrm{j}}$. $\mathrm{O}$ critério usual do método simplex é selecionar o menor custo reduzido dado por:

$$
\min _{\mathbf{j}} \overline{\mathbf{c}}_{\mathrm{j}}^{\pi}=\min \left(\overline{\mathbf{c}}_{\mathrm{j}}-\pi \mathbf{P}_{\mathbf{j}}-\pi_{0}\right)
$$

Substituindo (3.21) obtem-se:

$$
\min \overline{\mathbf{c}}_{\mathrm{j}}^{\pi}=\min \sum\left(\mathbf{c}^{\mathrm{k}}-\pi \mathbf{D}^{\mathrm{k}}\right) \overline{\mathbf{x}}_{\mathrm{j}}^{\mathrm{k}}-\pi_{0}
$$

Como $\overline{\mathbf{x}}_{\mathrm{j}}^{\mathrm{k}}$ é um ponto extremo de $\mathrm{X}^{\mathrm{k}}$ e a solução de um problema linear, cujo conjunto de restrições é convexo e limitado, ocorre sempre para um ponto extremo. Logo o mínimo em (3.24) é equivalente ao subproblema:

$$
\begin{array}{ll}
\text { Minimizar } & \sum\left(\mathbf{c}^{\mathrm{k}}-\pi \mathbf{D}^{\mathrm{k}}\right) \overline{\mathbf{x}}_{\mathbf{j}}^{\mathrm{k}} \\
\text { sujeito a } & \mathbf{A \mathbf { x } ^ { \mathrm { k } }}=\mathbf{b}^{\mathrm{k}} \mathbf{k}=1, \ldots, \mathrm{K} \\
\mathbf{0} & \leq \mathbf{x}^{\mathrm{k}} \leq \mathbf{u}^{\mathrm{k}}
\end{array}
$$

Como a função objetivo em (3.25) é aditivamente separável em $\overline{\mathbf{x}}_{\mathrm{j}}^{\mathrm{k}}$ e as restrições sobre $\overline{\mathbf{x}}_{\mathrm{j}}^{\mathrm{k}}$ são independentes para cada $\mathrm{k}$, o problema acima reduz-se a $\mathrm{K}$ subproblemas independentes na forma:

$$
\begin{aligned}
& \text { Minimizar }\left(\mathbf{c}^{\mathrm{k}}-\pi \mathbf{D}^{\mathrm{k}}\right) \overline{\mathbf{x}}_{\mathbf{j}}^{\mathrm{k}} \\
& \text { sujeito a } \quad \mathbf{A \mathbf { x } ^ { \mathrm { k } }}=\mathbf{b}^{\mathrm{k}} \\
& \mathbf{0} \leq \mathbf{x}^{\mathrm{k}} \leq \mathbf{u}^{\mathrm{k}}
\end{aligned}
$$


Portanto, para calcular o menor custo reduzido associado a variável não-básica $\lambda_{\mathrm{j}}$, deve-se resolver K subproblemas dados por (3.26), os quais são problemas de fluxo em rede de custo mínimo, não sendo necessário examinar todas as colunas não-básicas. Esta técnica é conhecida na literatura como geração de colunas.

Seja $P_{j^{*}}=\left(\overline{\mathbf{x}}_{\mathrm{j}^{*}}{ }^{1}, \overline{\mathbf{x}}_{\mathrm{j}^{*}}{ }^{2}, \ldots, \overline{\mathbf{x}}_{\mathrm{j}^{*}}{ }^{\mathrm{K}}\right)$ um ponto extremo ótimo para (3.25), com valor ótimo da função objetivo dado por $Z(\pi)$.

Portanto, se $\overline{\mathbf{c}}_{\mathrm{j}}^{\pi}=\mathrm{Z}(\pi)-\pi_{0}<0$, então, $\lambda_{\mathbf{j}^{*}}$ é a candidata a entrar na base do problema mestre, cuja coluna associada é dada como :

$$
\mathbf{P}_{j^{*}}=\left[\begin{array}{c}
\sum_{\mathbf{k}}\left(\mathbf{D}^{\mathrm{k}} \overline{\mathbf{x}}_{\mathbf{j}}^{\mathrm{k}}\right) \\
1
\end{array}\right] .
$$

Um algoritmo de dois níveis para a solução de (3.17) pode ser formulado, com o problema mestre sobre um segundo nível e os subproblemas (3.26) sobre um primeiro nível.

Algoritmo de Decomposição: Assume-se que uma base para o problema mestre (3.22) está disponível e os multiplicadores $\left(\pi, \pi_{0}\right)$ associados.

Passo 1: Usando os multiplicadores de Lagrange resolva os subproblemas (3.26) obtendo $\mathrm{P}_{\mathrm{j}^{*}}=\left(\overline{\mathbf{x}}_{\mathrm{j}^{*}}{ }^{1}(\pi), \overline{\mathbf{x}}_{\mathrm{j}^{*}}{ }^{2}(\pi), \ldots, \overline{\mathbf{x}}_{\mathrm{j}^{*}}{ }^{\mathrm{K}}(\pi)\right)$ com valor ótimo dado por $Z_{\mathrm{k}}{ }^{*}(\pi)$.

Passo 2: Calcule $\min \overline{\mathbf{c}}_{\mathrm{j}}^{\pi}=\sum_{\mathrm{k}=1}^{\mathrm{K}} Z_{\mathrm{k}} *(\pi)-\pi_{0}$

Se min $\overline{\mathbf{c}}_{\mathbf{j}}^{\pi} \geq 0$. Pare solução corrente ótima, faça $\mathbf{x}=\sum_{\text {basica }} \mathbf{P}_{\mathbf{j}} \wedge \lambda_{j}$ onde $\mathrm{P}_{\mathrm{j}^{*}}=\left(\overline{\mathbf{x}}_{\mathrm{j}^{*}}{ }^{1}, \overline{\mathbf{x}}_{\mathrm{j}^{*}}{ }^{2}, \ldots, \overline{\mathbf{x}}_{\mathrm{j}^{*}}{ }^{\mathrm{K}}\right)$ ponto extremo para $\mathrm{S}$.

Passo 3 : Se $\min \overline{\mathbf{c}}_{\mathbf{j}}^{\pi} \leq 0$, forme a coluna dada por (3.27).

Atualize $\mathrm{B}$, obtendo uma nova matriz básica.

Calcule novos multiplicadores e retorne ao passo 1 .

Observe que a solução para (3.17), não é necessariamente, uma das soluções propostas pelos subproblemas. Por (3.19), $\mathbf{x}=\left(\mathbf{x}^{1}, \mathbf{x}^{2}, \ldots, \mathbf{x}^{\mathrm{K}}\right)$ é uma combinação de algum número de tais soluções. Portanto, o problema mestre não pode obter uma solução ótima para (3.17) somente enviando multiplicadores para os subproblemas, mas combinando as soluções obtidas pelos subproblemas.

Para o problema mestre formulado em (3.22), a decomposição de Dantzig-Wolfe resolve problemas de otimização sobre o primeiro nível, mas não sobre o segundo, onde somente uma única trocas de colunas é feita. 
O problema mestre (3.22) pode ser formulado de modo que, em ambos os níveis se resolvam problemas lineares, da seguinte maneira:

$$
\begin{array}{ll}
\text { Minimizar } & \sum_{\text {jbasica }} \overline{\mathbf{c}}_{\mathrm{j}} \lambda_{\mathrm{j}}+\overline{\mathbf{c}}_{\mathrm{j}^{*}} \lambda_{\mathrm{j}^{*}} \\
\text { sujeito a } & \sum_{\mathrm{jbasica}} \mathbf{P}_{\mathrm{j}} \lambda_{\mathrm{j}}+\mathbf{P}_{\mathrm{j}^{*}} \lambda_{\mathrm{j}^{*}}=\mathbf{r} \\
& \sum_{\text {jbasica }} \lambda_{\mathrm{j}}+\lambda_{\mathrm{j}^{*}}=1 \\
\lambda_{\mathrm{j}} \geq 0 \mathrm{j}=1, \ldots, \mathrm{n}+1 \text { e } \lambda_{\mathrm{j}^{*}} \geq 0
\end{array}
$$

onde $\lambda_{\mathrm{j}} \mathrm{j}=1, \ldots, \mathrm{n}+1$ são as variáveis básicas corrente e $\lambda_{\mathrm{j}^{*}}$ é a variável entrando na base. Este problema é chamado problema mestre restrito.

Observe que a cada iteração da decomposição um problema mestre restrito é formado.

Como a variável entrando na base tem um custo reduzido negativo $\left(\overline{\mathbf{c}}_{\mathbf{j}}^{\pi}<0\right)$. Se a base é não-degenerada a variável saindo terá um custo reduzido positivo, e a nova solução é ótima para o problema 3.28.

Existem um número de maneiras, na qual o problema (3.17) pode ser decomposto. Cada uma delas afeta a forma do problema mestre e do problema mestre restrito.

Uma outra forma de decompor o problema (3.17) é considerar o conjunto dado por $\mathrm{X}^{\mathrm{k}}=\left\{\mathbf{x}^{\mathrm{k}}: \mathbf{A} \mathbf{x}^{\mathrm{k}}=\mathbf{b}^{\mathrm{k}}, \mathbf{0} \leq \mathbf{x}^{\mathrm{k}} \leq \mathbf{u}^{\mathrm{k}}\right\}$ para cada produto $\mathrm{k}$.

Como o conjunto $\mathrm{X}^{\mathrm{k}}$ para todo $\mathrm{k}$, é um conjunto compacto e convexo. Então, existem $x_{1}^{k}, \ldots, x_{n^{k}}^{k}$ pontos extremos de $X^{k}$, tal que, para qualquer ponto $x^{k} \in X^{k}$ existe um vetor $\lambda^{k}$ com $\mathrm{n}^{\mathrm{k}}$ componentes $\left(\mathrm{n}^{\mathrm{k}} \geq 1\right)$, tal que:

$$
\mathbf{x}^{\mathrm{k}}=\sum_{\mathrm{j}=1}^{\mathrm{n}^{\mathrm{k}}} \mathbf{y}_{\mathrm{j}}^{\mathrm{k}} \lambda_{\mathrm{j}}^{\mathrm{k}}, \text { onde } \sum_{\mathrm{j}=1}^{\mathrm{n}^{\mathrm{k}}} \lambda_{\mathrm{j}}^{\mathrm{k}}=1, \forall \mathrm{k} \text { e } \lambda_{\mathrm{j}}^{\mathrm{k}} \geq 0 \forall \mathrm{j}, \mathrm{k} .
$$

Adicionando as variáveis de folgas para as restrições de capacidade mútua do problema (3.17) e usando a representação para $\mathbf{x}^{\mathrm{k}}$ dada acima o problema em (3.17) é equivalente ao seguinte problema:

$$
\begin{array}{ll}
\text { Minimizar } & \sum_{j, k}\left(\mathbf{c}^{k} \mathbf{x}_{j}^{k}\right) \lambda_{j}^{k} \\
\text { sujeito a } & \sum_{j, k} \mathbf{D}^{k} \mathbf{x}_{j}^{k} \lambda_{j}^{k}+\mathbf{I s}=\mathbf{r} \\
& \sum_{j=1}^{n^{k}} \lambda_{j}^{k}=1, k=1, \ldots, K \\
& \lambda_{j}^{k} \geq 0 \quad k=1, \ldots, K \text { e } \mathbf{s} \geq \mathbf{0}
\end{array}
$$

Considere para cada $k=1, \ldots, K$ em (3.30), $\left\{\begin{array}{l}\mathbf{P}_{j}^{k}=\mathbf{D}^{k} \mathbf{y}_{j}^{k} \\ f_{j}^{k}=c^{k} \mathbf{y}_{j}^{k}\end{array}\right.$. 
Reescrevendo o problema (3.35) em termos de $\mathbf{P}_{\mathrm{j}}^{\mathrm{k}}$ e $\mathbf{c}_{\mathrm{j}}^{\mathrm{k}}$, tem-se o seguinte problema:

$$
\begin{array}{ll}
\text { Minimizar } & \sum_{\mathrm{j}, \mathrm{k}} \mathrm{f}_{\mathrm{j}}^{\mathrm{k}} \lambda_{\mathrm{j}}^{\mathrm{k}} \\
\text { sujeito a } \quad & \sum_{\mathrm{j}, \mathrm{k}} \mathbf{P}_{\mathrm{j}}^{\mathrm{k}} \lambda_{\mathrm{j}}^{\mathrm{k}}+\mathbf{I s}=\mathbf{r} \\
& \sum_{\mathrm{j}} \lambda_{\mathrm{j}}^{\mathrm{k}}=1 \quad \mathrm{k}=1, \ldots, \mathrm{K} \\
\lambda_{\mathrm{j}}^{\mathrm{k}} \geq 0, \mathrm{k}=1, \ldots, \mathrm{K} \quad \mathrm{s} \geq \mathbf{0}
\end{array}
$$

Este problema é chamado problema mestre ou problema extremo A matriz dos coeficientes do problema (3.32) acima é dado por:

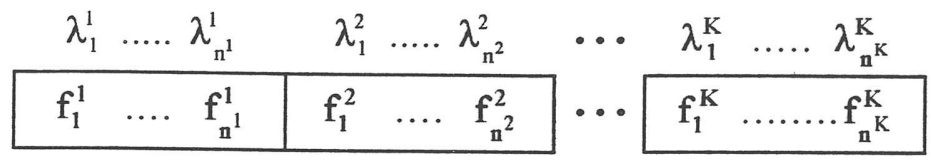

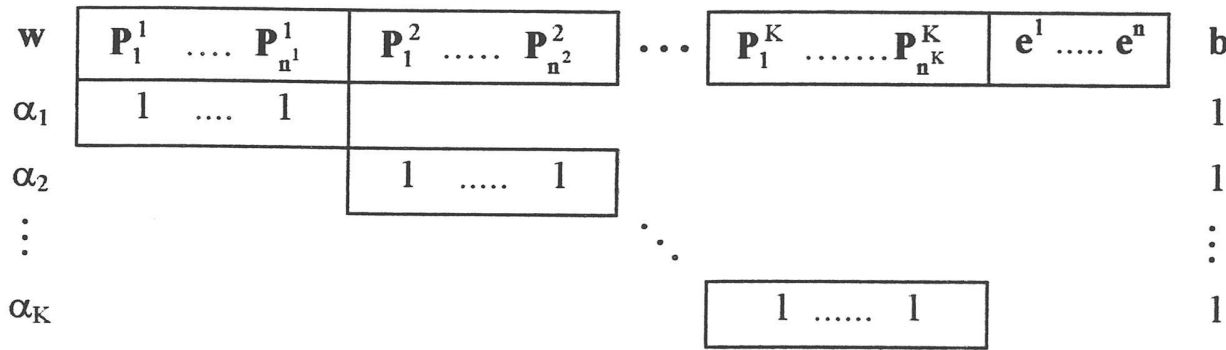

Figura 3.2 - Matriz dos coeficientes do Problema Mestre

onde e é um vetor unitário com n componentes, com "1" na i-ésima componente e zero nas demais.

O problema mestre (3.32) tem $n+\mathrm{K}$ restrições. Portanto, devem existir $n+\mathrm{K}$ colunas (vetores com $n+K$ componentes correspondendo aos pontos extremos) que constitua uma base factível, isto é, essas colunas são linearmente independentes.

Seja $\mathbf{B}(n+\mathrm{K} \times n+\mathrm{K})$ uma matriz básica factível para o problema (3.32) e $\mathbf{f}^{\mathrm{B}}$ o custos das variáveis associados a estas colunas básicas. Observe que uma base factível deve conter pelo menos uma coluna $\mathbf{P}_{j}^{k}$ para cada $k$. A solução básica não-negativa associada a esta base satisfazendo as restrições (3.32b) e (3.32c) é obtida atribuindo-se valor nulo para as variáveis $\left(\lambda_{j}^{\mathbf{k}}\right)$ associadas as colunas não-básicas. Se esta base não é ótima, uma coluna não-básica deve ser selecionada para entrar na base $\mathbf{B}$.

Seja $(\mathbf{w}, \alpha)$ o vetor de multiplicadores Lagrangeanos associadas as restrições conforme mostrado na matriz da figura 3.2 , onde $(\mathbf{w}, \alpha) \mathbf{B}=\mathbf{f}^{\mathrm{B}}$.

O custo reduzido para selecionar uma coluna não-básica para entrar na base, dado por:

$$
\overline{\mathbf{c}}_{\mathrm{j}}^{\mathrm{k}}=\mathbf{f}_{\mathrm{j}}^{\mathrm{k}}-(\mathbf{w}, \alpha)\left(\begin{array}{l}
\mathbf{P}_{\mathrm{j}}^{\mathrm{k}} \\
\mathbf{e}^{\mathrm{k}}
\end{array}\right)=\mathrm{f}_{\mathrm{j}}^{\mathrm{k}}-\mathbf{w} \mathbf{P}_{\mathrm{j}}^{\mathrm{k}}-\alpha_{\mathrm{k}} \text { correspondente as variáveis } \lambda_{\mathrm{j}}^{\mathrm{k}} \text {; e }
$$


$\overline{\mathbf{c}}_{\mathbf{j}}^{\mathrm{k}}=0-(\mathbf{w}, \alpha)\left(\begin{array}{l}\mathbf{e}^{\mathrm{j}} \\ 0\end{array}\right)=-w_{\mathbf{j}}$ correspondente as variáveis de folga $\mathrm{s}_{\mathrm{j}}$.

As condições para a otimalidade são dadas por:

(i) $\overline{\mathbf{c}}_{\mathbf{j}}^{\mathbf{k}}=\mathbf{f}_{\mathrm{j}}^{\mathbf{k}}-\mathbf{w} \mathbf{P}_{\mathbf{j}}^{\mathbf{k}}-\alpha_{\mathrm{k}} \geq 0, \mathrm{k}=1, \ldots, \mathrm{K}$;

(ii) $\bar{c}_{\mathrm{j}}^{\mathrm{k}}=-\mathrm{w}_{\mathrm{j}} \geq 0 \Rightarrow \mathrm{w}_{\mathrm{j}} \leq 0$,

então, a solução corrente associada a base B é ótima.

Da mesma forma que a anterior, se as condições (i) e (ii) não forem satisfeitas, o método simplex seleciona uma coluna não-básica correspondente $\lambda_{j}^{k}$ ou $s_{j}$ para entrar na base.

Considere $\overline{\mathbf{c}}_{\mathrm{j}}^{\mathrm{k}}=\mathrm{f}_{\mathrm{j}}^{\mathrm{k}}-\mathbf{w} \mathbf{P}_{\mathrm{j}}^{\mathrm{k}}-\alpha_{\mathrm{k}}$ o custo reduzido associado a variável $\lambda_{\mathrm{j}}^{\mathrm{k}}$. Portanto, o menor custo reduzido associado a $\lambda_{j}^{k}$ é dado por:

$$
\min _{k} \min _{j}\left(\mathbf{f}_{j}^{k}-\mathbf{w P}_{j}^{k}-\alpha_{k}\right) \text {. }
$$

Para k fixado, o problema de encontrar o menor custo reduzido é dado por:

$$
\min _{j}\left(\mathbf{f}_{j}^{k}-\mathbf{w P}_{j}^{k}-\alpha_{k}\right) \text {. }
$$

Substituindo (3.31) na equação acima obtem-se:

$$
\min _{j}\left(\left(c^{k}-w D^{k}\right) y_{j}^{k}-\alpha_{k}\right) \text {. }
$$

que é equivalente ao subproblema:

$$
\begin{array}{ll}
\text { Minimizar }\left(\mathbf{c}^{\mathrm{k}}-\mathbf{w} \mathbf{D}^{\mathrm{k}}\right) \mathbf{y}^{\mathrm{k}} & \\
\text { sujeito a } & \mathbf{A} \mathbf{y}^{\mathrm{k}}=\mathbf{b}^{\mathbf{k}} \\
& \mathbf{0} \leq \mathbf{y}^{\mathbf{k}} \leq \mathbf{u}^{\mathrm{k}} \text {, para cada } \mathrm{K}
\end{array}
$$

Este subproblemas são os mesmos que obtido anteriormente. Seja $x_{j^{*}}^{\mathbf{k}} \circ$ ponto extremo ótimo para o subproblema (3.35) com o valor da função objetivo ótimo dado por $\mathrm{z}^{\mathrm{k}}(\mathbf{w})$. Portanto, se $z^{k}(w)-\alpha_{k}<0$, então $\lambda_{j^{\prime \prime}}^{k}$ é uma candidata a entrar na base do problema mestre, caso contrário nenhum ponto extremo de $\mathrm{X}^{\mathrm{k}}$ é candidato a entrar na base.

Portanto, para calcular o custo reduzido deve-se resolver K subproblemas dados por (3.35) os quais são problemas de fluxo em redes de custo mínimo, não sendo necessário examinar todas as colunas não-básicas, mas apenas um subconjunto delas e que ainda podem ser geradas quando necessário.

Entre as candidatas a entrar na base associadas as variáveis $\lambda_{j}^{\mathrm{k}}$, com custo reduzido negativo, a selecionada é aquela com menor custo reduzido, ou seja, $\min _{1 \leq k \leq K}\left(z^{k}(w)-\alpha_{k}\right)$.

Se a nova coluna selecionada para entrar na base está associada a $\lambda_{j^{*}}^{k}$, ela é dada como:

$$
\mathbf{P}_{j^{*}}^{k}=\left[\begin{array}{l}
\sum_{k} \mathbf{D}^{k} \mathbf{x}_{j^{*}}^{k} \\
e^{k}
\end{array}\right] \text { com custo } f_{j}^{k}=c^{k} y_{j^{*}}^{k},
$$


onde $\mathbf{y}_{\mathbf{j}^{*}}^{\mathrm{k}}$ é a solução ótima de $z^{\mathrm{k}}(\mathbf{w})$.

Embora a coluna entrando $\left(\mathbf{P}_{\mathbf{j}^{*}}^{\mathrm{k}}\right)$ na base produza novos multiplicadores $(\mathbf{w}, \alpha)$, colunas semelhantes podem ter sido geradas pelas soluções de subproblemas de outros subsistemas e um número delas, provavelmente teria custo reduzido negativo usando os multiplicadores anteriores. Se qualquer uma destas colunas tem custo reduzido negativo usando os novos multiplicadores (o que pode acontecer com frequência, uma vez que os o conjunto de multiplicadores não difere muito a cada iteração), estas colunas podem ser usadas para reduzir o valor da função objetivo do problema mestre. Portanto, a cada iteração, todas as colunas geradas pela solução de cada um subproblemas, com custo reduzido negativo pode ser introduzida na base do problema mestre.

A nova formulação para o problema mestre é a seguinte:

$$
\begin{aligned}
& \text { Minimizar } \sum_{\mathrm{k}=1 \mathrm{~J}}^{\mathrm{K}} \sum_{\text {basica }} \mathrm{f}_{\mathrm{j}}^{\mathrm{k}} \lambda_{\mathrm{j}}^{\mathrm{k}}+\sum_{\mathrm{k}=1}^{\mathrm{K}} \mathrm{f}_{\mathrm{j}^{*}}^{\mathrm{k}} \lambda_{\mathrm{j}^{*}}^{\mathrm{k}} \\
& \text { sujeito a } \quad \sum_{\mathrm{k}=1 \mathrm{~J} \text { basica }}^{\mathrm{K}} \sum_{\mathrm{j}} \mathbf{P}_{\mathrm{j}}^{\mathrm{k}}+\sum_{\mathbf{k}=1}^{\mathrm{k}} \mathbf{P}_{\mathrm{j}^{*}}^{\mathrm{k}} \lambda_{\mathrm{j}^{*}}^{\mathrm{k}}=\mathbf{r} \\
& \sum_{\mathrm{J} \text { basica }}^{\mathrm{K}} \lambda_{\mathrm{j}}^{\mathrm{k}}+\lambda_{\mathrm{j}^{*}}^{\mathrm{k}}=1 \text { para } \mathrm{k}=1, \ldots, \mathrm{K} \\
& \lambda_{\mathrm{j}}^{\mathrm{k}} \geq 0 \text { e } \lambda_{\mathrm{j}^{*}}^{\mathrm{k}} \geq 0 \text { para } \mathrm{k}=1, \ldots, \mathrm{K},
\end{aligned}
$$

onde $\mathbf{P}_{j^{*}}^{k}$ são as colunas geradas pelas soluções dos subproblemas anteriores e $\mathbf{P}_{j}^{k}$ são as colunas da base corrente. $\mathrm{O}$ problema mestre (3.37) é chamado de problema mestre restrito.

Este problema mestre restrito difere do anterior (3.37), em dois aspectos: (i) o problema mestre (3.37) tem $\mathrm{K}$ restrições de convexidade uma para cada produto na rede, e cada um dos subsistemas são representados por um conjunto de variáveis $\lambda_{j}^{k} \geq 0$. Entretanto, como cada coluna não-básica com custo reduzido negativo é introduzida na base, pode-se esperar um decréscimo maior no valor da função objetivo do problema para o problema (3.37). Logo, pode-se esperar um número de iterações menor para o problema mestre restrito (3.37) do que para o problema mestre restrito (3.32). Observe que o problema (3.37) pode ser resolvido pelo método Generalized Upper Bounding.

Para os problemas de multifluxo apresentados, o fluxo dos produtos na rede possuem restrições de capacidade individual $\left(0 \leq \mathbf{x}^{\mathrm{k}} \leq \mathbf{u}^{\mathrm{k}}\right)$. No desenvolvimento da decomposição Dantzig-Wolfe, os subconjuntos $X^{k}=\left\{\mathbf{A} \mathbf{x}^{\mathbf{k}}=\mathbf{b}^{\mathbf{k}}\right.$ e $\left.\mathbf{0} \leq \mathbf{x}^{\mathrm{k}} \leq \mathbf{u}^{\mathbf{k}}\right\}$, com $\mathbf{u}^{\mathbf{k}}$ finito eram limitados.

Entretanto, se este não é o caso, quarido a decomposição Dantzig-Wolfe é aplicada, uma solução ilimitada pode ser obtida a partir da solução de algum dos subproblemas (3.35), dada por :

$$
\mathbf{y}_{j}^{k} \geq \mathbf{0}, A y_{j}^{k}=\mathbf{0} \quad \text { e } \quad\left(c^{k}-w^{k}\right) y_{j}^{k}<0 \text {, onde } y_{j}^{k} \text { é um raio extremo. }
$$

[Tomlin -66] especializou a decomposição de Dantzig-Wolfe para problemas de multifluxo em redes de custo mínimo de forma que os custos para os subproblemas sejam sempre 
não-negativos, isto é, $\left(\mathbf{c}^{\mathbf{k}}-\mathbf{w} \mathbf{D}^{\mathrm{k}}\right) \geq \mathbf{0}$, da seguinte maneira: se alguma componente $-\mathrm{w}_{\mathrm{i}}$ é negativa, a variável de folga pode ser introduzida na base.

\subsubsection{Algoritmo da decomposição de Dantzig-Wolfe}

\section{Inicialização}

Encontre uma base inicial para o problema mestre (3.42) e os multiplicadores correspondentes $(w, \alpha)$.

Se uma base factível não está disponível, adicione $n+K$ variáveis artificiais e aplique o método M-Grande.

\section{Teste da otimalidade}

Se qualquer $\mathrm{w}_{\mathrm{i}}>0$ então $\mathrm{S}_{\mathrm{i}}$ é uma candidata a entrar na base.

Senão considere os seguintes subproblemas $\mathrm{k}=1, \ldots, \mathrm{K}$

$$
\begin{aligned}
\operatorname{Minimizar} z^{k}(w)= & \left(c^{k}-w D^{k}\right) x^{k} \\
\text { sujeito a } & A x^{k}=b^{k} \\
& 0 \leq \mathbf{x}^{k} \leq \mathbf{u}^{k} .
\end{aligned}
$$

Se $z^{k}(w)-\alpha_{k}<0, \lambda_{j^{*}}^{k}$ é uma candidata para entrar na base do problema mestre Senão, termine, a solução corrente é ótima.

\section{Atualização da solução para o problema mestre}

Para $\mathrm{k}=1, \ldots, \mathrm{K}$ faça

$$
\text { Se } \mathrm{z}^{\mathrm{k}}(\mathbf{w})-\alpha_{\mathrm{k}}<0 \text { atualize a coluna escolhida, troque as colunas na base. }
$$

Retorne ao passo 1 com um novo conjunto de multiplicadores de Lagrange.

Como o algoritmo da decomposição Dantzig-Wolfe gera pontos factíveis a cada iteração, que fornecem um valor para a função objetivo, pode-se parar o algoritmo quando a diferença entre o valor da função objetivo em um ponto factível corrente e um limite inferior está dentro de um tolerância aceitável.

Proposição 3.7: Seja $\hat{\mathbf{c}}$ o valor da função objetivo para qualquer iteração do método simplex aplicado para o problema (3.22) com as variáveis duais $(\mathbf{w}, \alpha)$. Seja $\overline{\mathbf{x}}^{1}, \ldots, \overline{\mathbf{x}}^{\mathrm{k}}$ qualquer solução factível para o problema (3.22). Se $\mathbf{w} \leq \mathbf{0}$, então,

$$
\sum_{\mathrm{k}} \mathbf{c}^{\mathrm{k}} \overline{\mathbf{x}}^{\mathrm{k}} \geq \hat{\mathbf{c}}+\sum_{\mathrm{k}}\left(\mathrm{z}^{\mathrm{k}}(\mathbf{w})-\alpha^{\mathrm{k}}\right)
$$




\section{Capítulo 4}

\section{Impacto da Formulação sobre a Decomposiçãao Dantzig - Wolfe}

\subsection{Introdução}

Problemas de multifluxo em redes aparecem quando diversos itens compartilham a mesma rede, no sentido de que, alguns dos recursos existentes sobre esta rede estão limitados, portanto a utilização destes recursos pelos itens que percorrem a rede também são limitados.

Para o problema de multifluxo em redes apresentado nos capítulos anteriores, um produto foi definido como um item específico que percorre a rede, isto é, cada item com características físicas distintas define um produto. Esta definição para produto, gera uma formulação para o problema de multifluxo chamada de Problema do Produto Específico. Entretanto, essa não é a única definição para o termo produto.

O conjunto de produtos em uma rede pode ser dado como: (i) o fluxo de itens específicos que percorrem a rede; (ii) o fluxo de itens entre uma origem e destino comum; (iii) ou o fluxo de itens com um destino comum (ou uma origem comum); definidos de maneira única sobre a rede.

O número de produtos gerados é diferente em cada uma das definições anteriores. Portanto, definições distintas para o produto geram formulações distintas para o problema de multifluxo.

A prática comum sobre a decisão de uma formulação apropriada, tem sido a de escolher a formulação que produza o menor número de produtos, minimizando deste modo o tamanho do problema do multifluxo a ser resolvido.

A decomposição de Dantzig-Wolfe, descrita no capítulo anterior, consiste em rescrever o problema de multifluxo de forma equivalente em função dos pontos extremos dos subproblemas de fluxo em rede, que constituem o subconjunto de restrições bloco angular do problema. Portanto, para cada produto na rede um subproblema deve ser resolvido.

Neste capítulo apresentaremos um estudo feito por [JONES et al - 93], que investigaram o impacto da formulação quando a Decomposição de Danzig-Wolfe é utilizada para resolver problemas de multifluxo em rede. 


\subsection{As Três Formulação para problemas de multifluxo}

Considere o seguinte problema de multifluxo em redes

$$
\begin{array}{ll}
\text { Minimizar } & \sum_{k=1}^{\mathscr{K}} \mathbf{c}^{k} \mathbf{x}^{k} \\
\text { sujeito a } & \mathbf{A}^{k} \mathbf{x}^{k}=\mathbf{b}^{k} \quad k \in \mathscr{K} \\
& \sum_{k=1}^{\mathscr{K}} \mathbf{D}^{k} \mathbf{x}^{k} \leq \mathbf{r} \\
& \mathbf{x}^{k} \geq \mathbf{0} \quad k \in \mathscr{K}
\end{array}
$$

Este problema linear é uma formulação genérica para um problema de multifluxo em redes onde:

$\mathscr{K}$ : é o conjunto de produtos;

$\mathbf{A}^{k}$ : é a matriz incidência nó-arco para o produto $k$ sobre a rede $q=[\varkappa . A]$ com $m$ nós e $n$ arcos;

$\mathbf{x}^{k}$ : é o vetor de fluxo sobre os arco para o produto $k$;

$\mathbf{c}^{k}$ : é o vetor dos coeficientes dos custos para o produto $k$;

$\mathbf{b}^{k}$ : é o vetor de estoques/demandas requeridas para o produto $k$;

$\mathbf{r}$ : é o vetor de capacidade mútua dos arcos na rede para todos os produtos $k \in \mathscr{K}$;

$\mathbf{D}^{k}$ : é uma matriz diagonal de dimensões apropriadas, onde os elementos da diagonal são iguais a 1 se o arco correspondente na rede tem capacidade mútua, e tem valores iguais a zero caso contrário.

O problema dado em (4.1) apresenta uma estrutura bloco angular geral para um problema de multifluxo em rede, na qual o primeiro conjunto de restrições (4.1b) são as restrições de conservação de fluxo para cada produto $k \in \mathscr{K}$, e o segundo conjunto de restrições (4.1c) são as restrições de acoplamento chamadas de capacidade mútua. Para facilitar a discussão, os problemas de multifluxo apresentados neste capítulo não possuem restrições de capacidades individuais sobre cada produto.

Existem três maneiras diferentes para se definir um produto sobre uma rede. Cada uma delas geram formulações distintas para o problema de multifluxo em redes, onde em cada uma, um produto é definido de uma única maneira.

-Problema Produto Específico (PPE): Nesta formulação um produto é definido como um item específico que percorre a rede a partir de múltiplas origens para múltiplos destinos, onde o produto $k$ representa o item específico $\mathrm{k}$. Isto é, se na rude existem o fluxo de $\mathrm{K}$ itens distintos, $\mathrm{k}=1, \ldots, \mathrm{K}$, o número de produtos nesta formulação será igual ao número de itens. Esta foi a definição para produto utilizada nos capítulos anteriores.

-Problema Destino Especifico (PDE): Para esta formulação um produto é definido como um item que percorre a rede a partir de múltiplas origens para um destino específico $t$ (ou vice- 
versa, a partir de uma origem específica $s$ para múltiplos destinos) onde o produto $k$ é dado pelo par $(\mathrm{k}, \mathrm{t})$, que identifica o item $\mathrm{k}$ que percorre a rede a partir de múltiplas origens para um destino específico $t$.

-Problema Origem- Destino Específicos (POD): Nesta formulação, um produto é definido como um item que percorre a rede a partir de uma origem específica para um destino específico. Portanto, um produto $k$ é dado pela tripla $(\mathrm{k}, \mathrm{s}, \mathrm{t})$, onde $\mathrm{k}$ é o item $1 \leq \mathrm{k} \leq \mathrm{K}, s \in \mathrm{S}$ é a origem específica ( $\mathrm{S} \subseteq \mathscr{X}$ é o conjunto das origens específicas) e $t \in \mathrm{T}$ é o destino específico ( $\mathrm{T} \subseteq \boldsymbol{U}$ é o conjunto dos destinos específicos).

As diferenças entre as formulações são dadas pelo número de blocos das submatrizes $\mathbf{A}^{k}$ na matriz de restrições, pois cada problema tem $|\mathscr{K}|$ blocos e pela densidade do vetor de estoque/demanda $\mathbf{b}^{k}$.

Lembramos que em um problema geral de multifluxo em redes, cada produto que percorre a rede está sujeito às restrições de conservação de fluxo da rede.

É assumido que a rede é idêntica para as três formulações e para cada um dos produtos. Portanto a matriz incidência nó-arco $\mathbf{A}^{k}=\mathbf{A}$, para todo $k \in \mathscr{K}$.

A matriz de restrições para o problema de multifluxo geral é dada por:

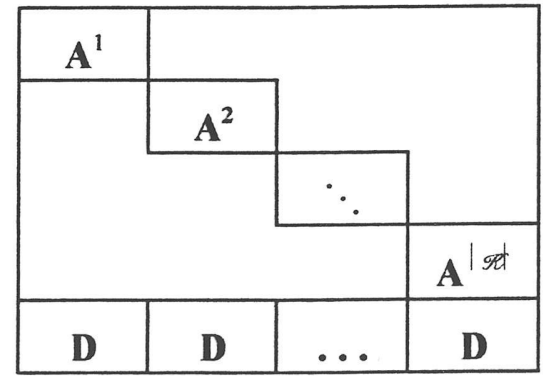

Figura 4.1 - Matriz de restrições para o problema 4.1

Como a rede é idêntica, o número de restrições em cada bloco $\mathbf{A}^{k}$ é o mesmo para cada uma das três formulações, mas o número de blocos varia de acordo com $|\mathscr{K}|$, devido aos aspectos combinatórios de todos os itens, origens e destinos.

$\mathrm{O}$ POD terá potencialmente um número maior de blocos de submatrizes $\mathbf{A}$ do que o $\mathrm{PDE}$ e o PPE. A matriz de restrições para o PDE terá um número maior de blocos de submatrizes $\mathbf{A}$, do que a matriz de restrições para o PPE.

Os vetores $\mathbf{b}^{k}$ variam significativamente entre as três formulações. Isto pode ser visto, investigando o número de elementos não-nulos para cada $\mathbf{b}^{k}$ para as três formulações dadas a seguir. 
-Para o problema do produto específico (PPE), cada produto $k$ corresponde a um item específico k, e no vetor de estoque/demanda existem múltiplos elementos não-nulos para as múltiplas origens e múltiplos destinos. $\mathrm{O}$ conjunto de produtos para esta formulação é dado por:

$$
\left.\mathscr{K}(\mathrm{PPE})=\{k=\mathrm{k}, 1 \leq \mathrm{k} \leq \mathrm{K}\} \text { e } \mathbf{b}^{k}=\left(\mathrm{b}_{1}{ }^{\mathrm{k}}, \mathrm{b}_{2}{ }^{\mathrm{k}}, \ldots, \mathrm{b}_{\mathrm{m}}{ }^{\mathrm{k}}\right)\right\}
$$

-Para o problema do destino especifico (PDE), cada produto $k$ correspondente ao par $(k, t)$, em cada vetor de estoque/demanda $\left(\mathbf{b}^{h}\right)$ existem múltiplos elementos não-nulos para as restrições de estoque correspondentes as múltiplas origens, mais um elemento não-nulo correspondente ao destino específico $t$. $\mathrm{O}$ conjunto de produtos é dado como:

$$
\begin{aligned}
\mathscr{K}(\mathrm{PDE}) & \left.=\{k=(\mathrm{k}, \mathrm{t}), 1 \leq \mathrm{k} \leq \mathrm{K} \text { e t } \in \mathrm{T}\} \text { e } \mathbf{b}^{k}=\left(\theta_{1}{ }^{k}, \theta_{2}{ }^{k}, \ldots, \theta_{\mathrm{m}}{ }^{k}\right)\right\} \\
\theta_{\mathrm{i}}^{k} & =\left\{\begin{array}{ll}
\mathrm{b}_{\mathrm{i}}^{\mathrm{k}} & \text { se } \mathrm{b}_{\mathrm{i}}^{\mathrm{k}}>0 \text { ou } \mathrm{i}=\mathrm{t} \\
0 & \text { caso contrario }
\end{array},\right.
\end{aligned}
$$

onde $\mathrm{T} \subseteq \boldsymbol{H}$. (note que $\mathrm{b}_{\mathrm{t}}{ }^{\mathrm{k}}<0$, pois $\mathrm{t}$ é um nó destino).

-No problema origem destino específico ( POD), cada produto $k$ correspondente à tripla $(\mathrm{k}, \mathrm{s}, \mathrm{t})$ e em cada vetor de estoque/demanda ( $\left.\mathbf{b}^{\mathrm{h}}\right)$ existem somente dois elementos não nulos para cada produto $k \in \mathscr{T} \xi$ um deles correspondente a origem específica $s$ e o outro correspondente ao destino específico $t$ do item $\mathrm{k}$. $\mathrm{O}$ conjunto dos produtos para essa formulação é dado por:

$$
\begin{aligned}
\mathscr{K}(\mathrm{PDO}) & \left.=\{k=(\mathrm{k}, \mathrm{s}, \mathrm{t}) 1 \leq \mathrm{k}<\mathrm{K}, \mathrm{s} \in S, \mathrm{t} \in T\} e \mathbf{b}^{k}=\left(\theta_{1}{ }^{k}, \theta_{2}{ }^{k}, \ldots, \theta_{\mathrm{m}}{ }^{k}\right)\right\} \\
\theta_{\mathrm{i}} & =\left\{\begin{array}{ll}
\mathrm{b}_{\mathrm{i}}^{\mathrm{k}} & \text { se } \mathrm{i}=\mathrm{s} \text { ou } \mathrm{t} \\
0 & \text { caso } \text { contrario }
\end{array} .\right.
\end{aligned}
$$

Para verificar estas diferenças entre as três formulações, considere a rede dada a seguir, onde existe um fluxo de dois itens, isto é, $\mathrm{k}=1,2$ com demandas e estoques dados na rede.

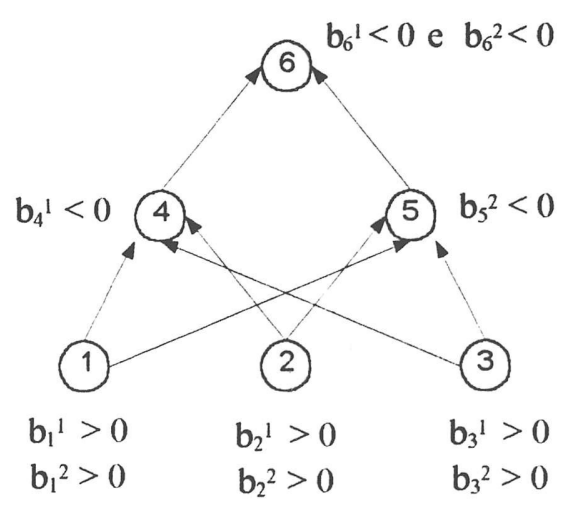

Figura 4.2: Exemplo de Rede com dois itens

onde $b_{i}{ }^{k}$ : é o estoque e/ou demanda do produto k no nó i. 
-Problema do Produto Especifico (PPE) tem-se: $\mathscr{K}(\mathrm{PPE})=\{k=1,2\}$.

$$
\mathbf{A} \mathbf{x}^{1}=\mathbf{b}^{1} \text {, onde } \mathbf{b}^{1}=\left[\begin{array}{c}
b_{1}^{1} \\
b_{2}^{1} \\
b_{3}^{1} \\
b_{4}^{1} \\
b_{5}^{1} \\
b_{6}^{1}
\end{array}\right], \quad \text { e } \quad A \mathbf{x}^{2}=\mathbf{b}^{2} \text {, onde } \mathbf{b}^{2}=\left[\begin{array}{c}
b_{1}^{2} \\
b_{2}^{2} \\
b_{3}^{2} \\
b_{4}^{2} \\
b_{5}^{2} \\
b_{6}^{2}
\end{array}\right] \text {, A é a matriz incidência nó-arco }
$$

para a rede da figura 4.2 A matriz de restrições para a formulação do PPE é dada por:

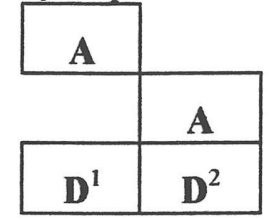

-Problema Destino Especifico (PDE) tem-se: $\mathscr{R}(\mathrm{PDE})=\{k=(\mathrm{k}, \mathrm{t}) ;(1,6),(1,4),(2,5),(2,6)\}$.

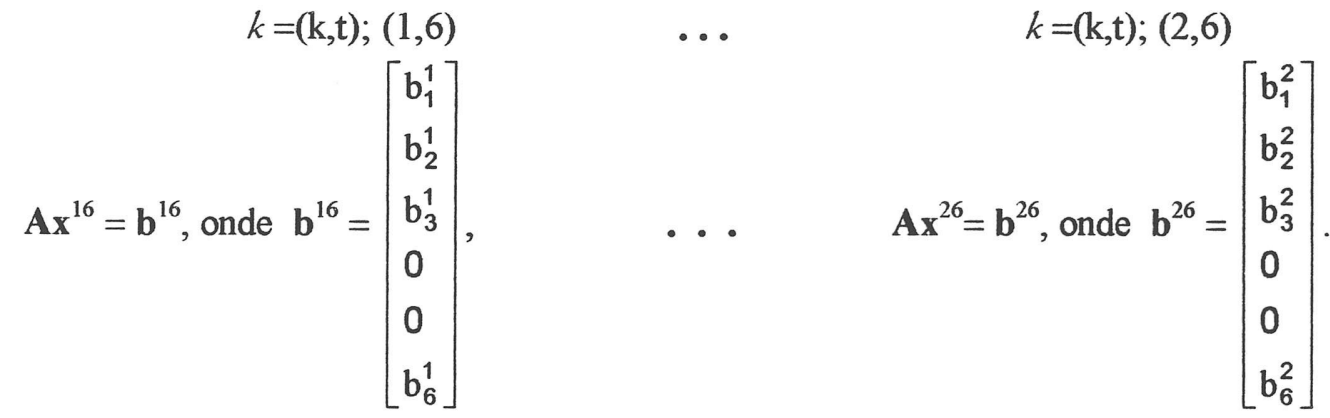

A matriz de restrições para esta formulação do PDE é da forma:

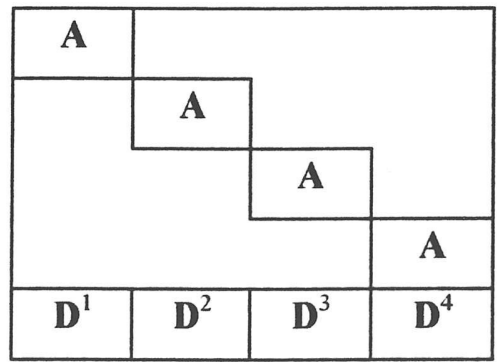

-Problema origem destino especifico (POD) tem-se:

$\mathscr{K}(\mathrm{PDO})=\{k=(\mathrm{k}, \mathrm{s}, \mathrm{t}) ;(1,1,4),(1,1,6)(1,2,4),(1,2,6),(2,1,5),(2,1,6),(2,3,4),(2,3,6)\}$

Para $k=(1,1,4)$,

$\mathbf{A x} \mathbf{x}^{114}=\mathbf{b}_{14}{ }^{1}$, onde $\mathbf{b}_{14}{ }^{1}=\left[\begin{array}{l}\mathbf{b}_{1}^{1} \\ 0 \\ 0 \\ \mathbf{b}_{4}^{1} \\ 0 \\ 0\end{array}\right]$,onde a matriz de restrições

para POD é dada ao lado.

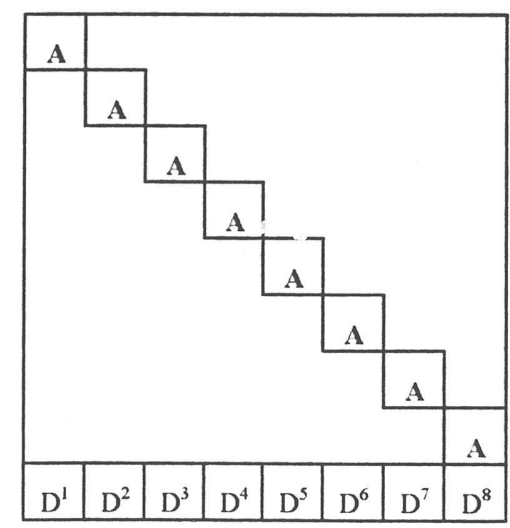


Portanto, na formulação para o (POD) a matriz de restrições possui um número maior de blocos formados pelas submatrizes A (matriz incidência nó-arco) do que para as formulações (PDE) e do (PPE). Entretanto, observe que o vetor $\mathbf{b}^{k}$ é muito mais esparso para o (POD) que para as outras duas formulações.

\subsection{A Decomposição Dantzig-Wolfe para as três formulações}

Nesta seção apresentaremos a formulação matemática geral para o problema mestre e os subproblemas quando a decomposição Dantzig-Wolfe é aplicada para resolver cada uma das três formulações do problema de multifluxo.

\subsubsection{A Formulação do Problema Mestre Restrito}

A decomposição de Dantzig-Wolfe, dada no capítulo anterior, considera o conjunto convexo das restrições bloco angulares (4.1b) do problema de multifluxo geral (4.1) limitado. Esse conjunto é composto por subconjuntos convexos de restrições independentes. Então, qualquer ponto que pertença a um destes subconjuntos convexos independentes (também limitados), pode ser escrito como combinação convexa das suas soluções pontos extremos dos subconjuntos.

Utilizando essa combinação convexa das soluções pontos extremos no problema de multifluxo geral (4.1), um problema mestre equivalente é formado.

O problema mestre deve ser apresentado de forma equivalente, para que na resolução da decomposição o problema mestre também seja resolvido. Este problema mestre equivalente ao anterior é chamado de problema mestre restrito. A formulação do problema mestre restrito apresentada a seguir é uma formulação geral para o problema geral de multifluxo apresentado em (4.1). O conjunto $\mathscr{K}$ será usado no problema mestre restrito para representar o conjunto de produtos para cada formulação.

Como a técnica de geração de colunas é utilizada no algorítmo da decomposição de Dantzig-Wolfe, existe um número variando de colunas associadas com cada $k \in \mathscr{K}$ no problema mestre restrito elaborado a cada iteração da decomposição. Seja $\mathrm{J}^{k}$ o número total de colunas no problema mestre restrito corrente, associado com o elemento $k$. O problema mestre restrito geral pode ser dado como: Minimizar $\sum_{k \in \mathscr{K}} \sum_{\mathbf{j} \in \mathrm{J}^{k}}\left(\mathbf{c}^{k} \mathbf{x}_{\mathbf{j}}^{k}\right) \lambda_{\mathbf{j}}^{k}$

$$
\begin{gathered}
\text { sujeito a } \quad \sum_{k \in \mathscr{K}} \sum_{\mathbf{j} \in \mathbf{J}^{k}}\left(\mathbf{D}^{k} \mathbf{x}_{\mathbf{j}}^{k}\right) \lambda_{\mathbf{j}}^{k}+\mathbf{I s}=\mathbf{r} \\
\sum_{\mathbf{j} \in \mathrm{J}^{k}} \lambda_{\mathbf{j}}^{k}=1 \quad k \in \mathscr{K} \\
\lambda_{\mathbf{j}}^{k} \geq 0 \text { para cada } \mathrm{j} \in \mathrm{J}^{\mathrm{k}}, k \in \mathscr{K},
\end{gathered}
$$


onde $\mathbf{x}_{\mathbf{j}}^{k}$ é a j-ésima solução ponto extremo do subproblema associado ao produto $k$.

As matrizes das restrições para o (POD), (PDE) e para (PPE) são semelhantes, isto é, a matriz incidência nó-arco é mesma para as três formulações, portanto o problema mestre para as três formulações também são semelhantes. Entretanto, o número de restrições no problema mestre é dado pelo número de restrições de capacidade mútua mais um número de restrições de convexidade, associadas as soluções pontos extremos dos subproblemas. Lembramos que o número de subproblemas é determinado pelo número de produtos na formulação.

Portanto, o número de restrições de convexidade para o problema mestre restrito pode ser muito diferente em cada formulação, dependendo da definição de $\mathscr{K}$.

Como o conjunto $\mathscr{K}(\mathrm{POD})$ tem muito mais elementos do que $\mathscr{R}(\mathrm{PDE})$ e do que $\mathscr{R}(\mathrm{PPE})$, conseqüentemente, terá muito mais restrições de convexidade no problema mestre restrito do que as outras duas formulações. E o conjunto $\mathscr{K}(\mathrm{PDE})$ tem mais elementos do que $\mathscr{R}(\mathrm{PPE})$. Logo, PDE também terá mais restrições de convexidade no problema mestre restrito do que o PPE.

\subsubsection{A formulação do Subproblemas para (POD), (PDE) e (PPE)}

Para cada iteração do algoritmo da decomposição Dantzig-Wolfe, um subproblema é resolvido para cada $k \in \mathscr{K}$. Para o problema de multifluxo (4.1), cada subproblema descreve um problema de fluxo em rede de um único produto.

O formulação para os subproblemas pode ser apresentada por:

$$
\begin{gathered}
\text { Minimizar }\left(\mathbf{c}^{k}-\mathbf{w D}^{k}\right) \mathbf{x}^{k} \\
\text { sujeito a } \mathbf{A} \mathbf{x}^{k}=\mathbf{b}^{k} \\
\qquad \mathbf{x}^{k} \geq \mathbf{0}
\end{gathered}
$$

onde os elementos do vetor $\mathbf{w}$ são os valores dos multiplicadores de Lagrange (variáveis duais) enviadas pelo problema mestre e que estão associadas as restrições de acoplamento (4.5b).

O problema (4.6) são problemas de fluxo em redes, onde as soluções variam de uma formulação para outra, sendo unicamente determinada por $\mathbf{b}^{k}$.

- Para a formulação do (PPE), $\mathbf{b}^{k}$ determina que o item $\mathrm{k}$ deve ser enviado a partir de múltiplas origens para múltiplos destinos. Neste caso, os subproblemas (4.6) que devem ser resolvidos a cada iteração, são problemas de fluxo em redes de custo mínimo, o qual foi apresentado no primeiro capítulo deste trabalho. Assim, o ponto extremo gerado por este subproblema é uma árvore de custo mínimo.

-Na formulação do (PDE), $\mathbf{b}^{k}$ contém múltiplos elementos positivos, correspondentes as múltiplas origens do item $\mathrm{k}$ e um único elemento negativo para a demanda do destino específico $\mathrm{t}$. 
Esses subproblemas são problemas de fluxo de rede do caminho mínimo com múltiplas origens. Portanto, a solução para cada um dos subproblemas é um vetor de fluxos sobre os arcos determinando uma árvore de caminhos mínimos provenientes de todos os nós estoques do item $\mathrm{k}$ para o nó destino específico t. A formulação deste subproblema foi apresentada em (1.6) também no primeiro capítulo.

- Para a formulação (POD), $\mathbf{b}^{k}$ é um vetor de estoques/demandas com somente dois elementos não-nulos, que correspondem as restrições de conservação sobre o fluxo entre uma origem específica e um destino específico. Portanto, os subproblemas para esta formulação, que são resolvidos a cada iteração, são problemas do caminho mínimo onde a solução para cada um dos subproblemas é um vetor de fluxo sobre os arcos determinando o caminho mínimo a partir do nó origem específico $s$ para o nó destino específico $t$ do item $\mathrm{k}$. A formulação para este subproblema foi apresentada em (1.4) no primeiro capítulo.

Analisando a densidade do vetor $\mathbf{b}^{k}$ pode-se observar que a solução ponto extremo obtida quando um subproblema para a formulação (POD) é resolvido, é mais esparsa do que a obtida pelos subproblemas do (PDE) e do (PPE). Pois a solução ponto extremo para o subproblema do POD representa o fluxo ao longo de um único caminho. Logo, a coluna adicionada no problema mestre tem um elemento igual a " 1 " correspondente a restrição de convexidade e todos os outros valores não nulos iguais a demanda $b_{s}{ }^{k}$. Enquanto que, a solução ponto extremo obtida a partir dos subproblemas de (PDE) e do (PPE) produzem um número variando de valores não-nulos dentro de cada coluna.

\subsection{Comparação das Formulações}

Como o conjunto $\mathscr{K}(\mathrm{POD})$ tem potencialmente, um número maior de elementos do que os conjuntos das outras formulações, isto é, do que $\mathscr{K}(\mathrm{PDE})$ e do que $\mathscr{T}(\mathrm{PPE})$, existem muito mais subproblemas e portanto, a cada iteração existe um número maior de colunas adicionadas ao problema mestre restrito da decomposição.

Entretanto, [Jones et al. -93] concluíram que para alguns tipos de redes, o número total de pontos extremos adicionados no problema mestre restrito para a formulação (POD) é menor do que o número de pontos extremos adicionados no problema mestre restrito de (PDE) ou do (PPE).

Considere $\mathrm{J}_{\mathrm{t}}{ }^{k}$ o número de pontos extremos associados com o prociuto $k \in \mathscr{K}$ adicionados ao problema mestre restrito até a iteração t. Defina $\wp_{\mathrm{t}}$ como o número máximo de colunas na decomposição para a iteração $\mathrm{t}$ associado com qualquer elemento $k \in \mathscr{K}$, isto é, $\wp_{\mathrm{t}}=\max { }_{k \in \mathscr{R}}\left\{\mathrm{J}_{\mathbf{t}}{ }^{k}\right\}$. 
Portanto, para todas as formulações, um limitante superior para as colunas no problema mestre restrito na iteração $\mathrm{t}$ é $|\mathscr{K}| \times \wp_{\mathrm{t}}$. [Jones et al. -93] fizeram extensivos experimentos computacionais com problemas de multifluxo em diferentes aplicações e concluíram que:

$$
|\mathscr{R}(\mathrm{POD})| \times \wp_{\mathrm{To}}(\mathrm{POD})<<|\mathscr{R}(\mathrm{PDE})| \times \wp_{\mathrm{Td}}(\mathrm{PDE})
$$

onde,

$\mathrm{T}_{0}$ é o número de iterações para a otimalidade do POD; $\mathrm{T}_{\mathrm{d}}$ é o número de iterações para a otimalidade do PDE; $|\mathscr{K}(\mathrm{POD})|$ é o número de produtos na formulação do POD; $|\mathscr{K}(\mathrm{PDE})|$ é o número de produtos na formulação do PDE; $\wp_{\mathrm{To}}(\mathrm{POD})$ é o número máximo de pontos extremos que um subproblema do POD pode gerar até a iteração $\mathrm{T}_{0} ; \wp_{\mathrm{Td}}(\mathrm{PDE})$ é o número máximo de pontos extremos que um subproblema do PDE pode gerar até a iteração $T_{d}$.

Isto pode ser verificado, investigando o número total de pontos extremos em cada uma das duas formulações, na rede dada a seguir.

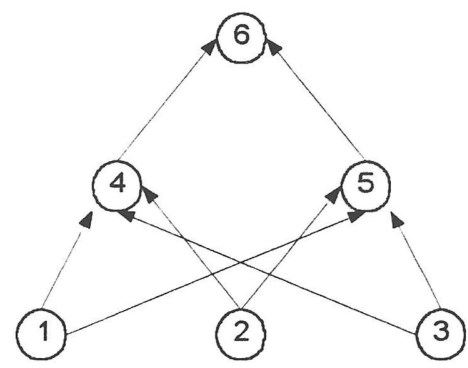

Figura 4.3a

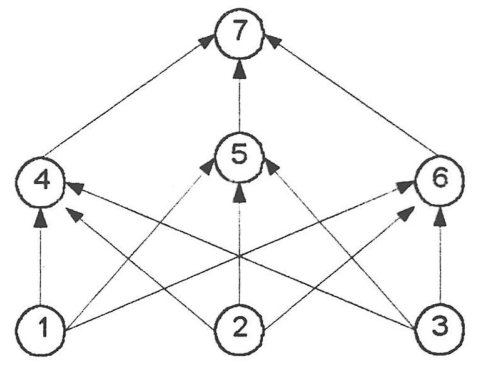

Figura $4.3 b$

Observe que na figura 4.3a para a formulação do POD esta rede possui 6 (seis) possiveis caminhos a partir de cada nó estoque $S=\{1,2,3\}$ para o único destino $t \in \mathrm{T}=\{6\}$ sendo que, para cada $(k, s, t)$ existem somente duas soluções caminhos possíveis. Para a formulação do PDE esta mesma rede possui 8 (oito) possíveis árvores solução. A figura $4.3 \mathrm{~b}$ mostra que a adição de um nó na rede aumenta o número de soluções caminhos de 6 para 9, enquanto que o novo número de soluções árvores aumentou de 8 para 27. Potanto, para essa pequena rede pode-se observar que o número de pontos extremos gerados por cada subproblema do POD é menor do que o número de pontos extremos que um subproblema do PDE pode gerar.

As diferenças entre a formulação baseada em caminhos do POD e entre as formulações baseadas em árvores do PDE e o PPE foram apresentadas. Entretanto, para um comparação válida entre as três formulações estes problemas devem ser matematicamente equivalentes, o que é suficiente mostrar que os subproblemas são equivalentes.

Adiante serão apresentadas algumas proposições que mostram a equivalência entre POD e o PDE. Entretanto, o exemplo dado a seguir demonstra que o POD não é equivalente ao PPE, 
comparando a solução ótima de um conjunto de problemas do caminho mínimo do POD e a solução ótima do PPE correspondente ao problema de fluxo em rede de custo mínimo.

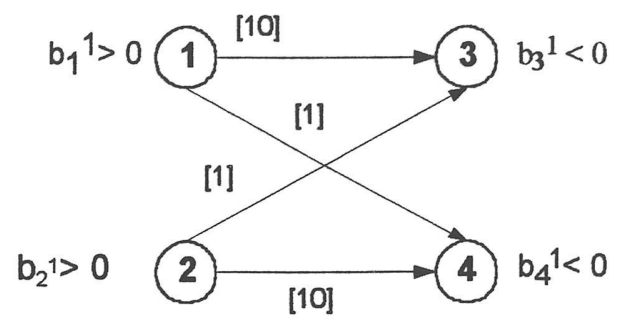

Figura 4.4 Exemplo da não validade dos corolários 4.2 e 4.3 para PPE

Para este exemplo da figura 4.4, o POD tem dois produtos definidos como $k=(\mathrm{k}, \mathrm{s}, \mathrm{t})$ onde $\mathscr{K}(\mathrm{POD})=\{(1,1,3),(1,2,4)\}$. A solução ótima para os dois suproblemas tem um custo total de 20 unidades, enviando o fluxo sobre os arcos $(1,3)$ e $(2,4)$. Como a formulação do PPE impõe somente conservação de fluxo agragados em cada nó, a solução resultante utiliza os arcos $(1,4)$ e $(2,3)$ com um custo de 2 unidades.

A equivalência entre POD e PDE é agora estabelecida através das seguintes proposições.

Proposição 4.1: Seja $\mathbf{x}^{\mathrm{kt}}$ uma solução factível para o PDE, então existe uma solução ótima para o POD dada por $\mathrm{x}^{\mathrm{kst}}$ para cada $\mathrm{s} \in S$ (conjunto das origens), $\mathrm{t} \in \mathrm{T}$ (conjunto dos destinos) e 1 $\leq \mathrm{k} \leq \mathrm{K}$, tal que;

$$
\mathbf{x}^{\mathrm{kt}}=\sum_{\mathrm{s} \in S} \mathbf{x}^{\mathrm{kst}}
$$

Este lema permite que os fluxos agregados em uma estrutura árvore a partir de todos os nós estoques para um destino t, pode ser decomposto em um conjunto de caminhos a partir de um nó estoque específico para o nó destino em questão.

Proposição 4.2: Se os custos sobre os arcos na rede não dependem da origem ou destino dos produtos e $\mathrm{x}^{\mathrm{kst}}$ é ótima para o POD, então $\mathrm{x}^{\mathrm{kt}}$ dado em (4.8) é ótima para o PDE.

Proposição 4.3: Se os custos sobre os arcos na rede não dependem de sua origem ou destino e $\mathrm{x}^{\mathrm{kt}}$ é ótima para o PDE, então uma solução ótima para $\mathrm{x}^{\mathrm{kst}}$ para o POD pode ser construida a partir da solução ótima $\mathrm{x}^{\mathrm{kt}}$ tal que (4.8) seja válida.

Portanto, quando a decomposição de Dantzig-Wolfe é utilizada para resolver a formulação do POD, as proposições anteriores 4.2 e 4.3 permitem que as soluções pontos extremos do POD sejam obtidos através das soluções árvores do caminho mínimo ótima dos subproblemas da formulação PDE e vice e versa.

Logo, o número de subproblemas que devem ser resolvidos a cada iteração da decomposição de Dantzig-Wolfe para a formulação POD pode ser reduzido, sendo necessário resolver apenas 
$|\mathscr{K}(\mathrm{PDE})|$ e decompondo as soluções desta árvore em caminhos ótimos para os pontos extremos correspondentes no POD.

A figura dada a seguir ilustra um exemplo de decomposição de fluxo para as formulações POD e PDE.

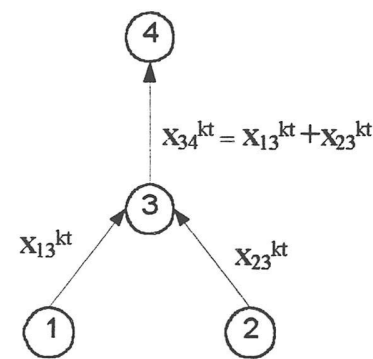

Figura 4.5a: Solução para o PDE

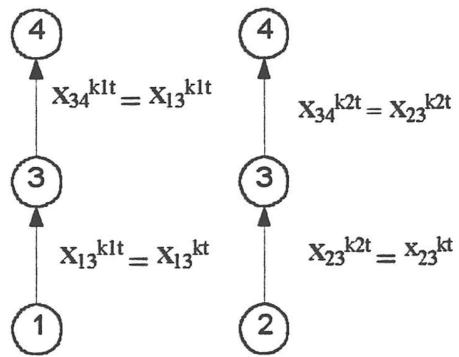

Figura 4.5b: Solução para o POD

\subsection{Uma formulação baseada em caminhos para o PPE}

As proposições 4.2 e 4.3 não são válidas para uma comparação direta das formulações baseada em árvores do PDE e do PPE, mesmo quando os custos sobre os arcos na rede não dependem de sua origem ou destino. Ou seja, uma solução ótima para o subproblema do PPE não pode ser construída agregando-se as soluções dos subproblemas do PDE e portanto, nem através das soluções dos subproblemas do POD.

Entretanto, o PPE pode ser transformado em uma formulação equivalente baseada em caminhos.

Considere a rede do PPE $\mathcal{G}=[\boldsymbol{n}, \mathscr{A}]$ com $m$ nós e $n$ arcos e uma ampliação dada por: $\hat{\mathfrak{g}}=[\hat{\boldsymbol{\eta}}, \hat{\boldsymbol{A}}]$, onde $\hat{\boldsymbol{\eta}}=\boldsymbol{\eta} \cup \hat{S} \cup \hat{T}$, com $\hat{S}=\left\{\hat{\mathrm{s}}_{1}, \hat{\mathrm{s}}_{\mathrm{s}}, \ldots, \hat{\mathrm{s}}_{\mathrm{K}}\right\}$ chamados de nós super-estoques e $\hat{T}=\left\{\hat{\mathrm{t}}_{1}, \hat{\mathrm{t}}_{2} \ldots, \hat{\mathrm{t}}_{\mathrm{K}}\right\} \quad$ chamados de nós e $\hat{\mathscr{A}}=\mathscr{A} \cup\{(\hat{\mathrm{s}}, \mathrm{s}): \hat{\mathrm{s}} \in \hat{S}, \mathrm{~s} \in S\} \cup\{(\mathrm{t}, \hat{\mathrm{t}}): \mathrm{t} \in T, \hat{\mathrm{t}} \in \hat{T}\}$, e $\mathrm{S}$ e $\mathrm{T}$ são os conjuntos originais de estoques e demandas.

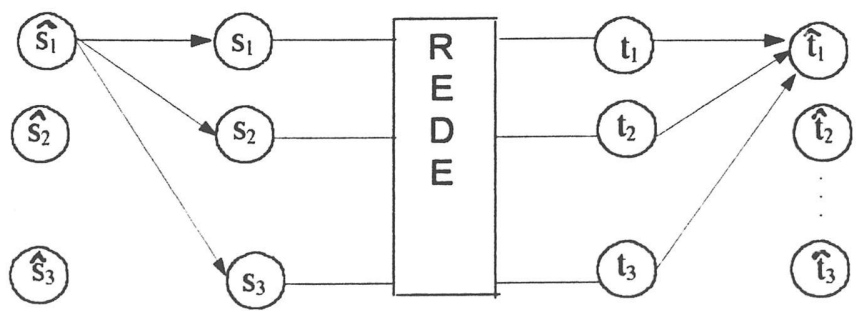

Figura 4.6: Ampliação da rede para o PPE

Para que este problema encontre uma solução caminho, isto é, que o fluxo do produto $k=\mathrm{k}$ percorra a rede a partir de seu nó super-estoque $\hat{s}_{\mathrm{k}}$ para o nó super-demanda $\hat{\mathrm{t}}_{\mathrm{k}}$ é necessário que no vetor de estoque/demanda tenha somente dois elementos não-nulos, sendo um correspondente ao nó 
super-estoque $\hat{s}_{\mathbf{k}}$ e um outro correspondente ao nó super-demanda $\hat{t}_{\mathbf{k}}$. Este problema é chamado de Problema Produto Específico Ampliado (PPEA).

Para que o Problema Produto Especifico Ampliado (PPEA) seja equivalente PPE, o fluxo total a partir do nó superestoque do produto $k=\mathrm{k}$ para o nó superdemanda deve ser tal que:

(i) $\hat{b}_{\mathrm{s}_{\mathrm{k}}}^{\mathrm{k}}=\sum_{\mathrm{s} \in S} \mathrm{~b}_{\mathrm{s}}^{\mathrm{k}}=\sum_{\mathbf{t} \in T} \mathrm{~b}_{\mathrm{t}}^{\mathbf{k}}$

onde $\mathrm{b}_{\mathrm{s}}{ }^{\mathrm{k}}$ é o estoque do produto $\mathrm{k}$, disponível para o nó estoque original $s ; \mathrm{b}_{\mathrm{t}}{ }^{\mathrm{k}}$ é a demanda do produto $\mathrm{k}$ para o nó original $\mathrm{t}$, garantindo que os estoques disponíveis nos nós super-estoques satisfaçam todos os estoques originais e que a rede está balanceada;

(ii) os fluxos sobre os novos arcos correspondente aos arcos a partir dos nós super-estoques para os nós estoques originais, e o fluxo nos arcos a partir dos nós demandas originais para os nós super-demandas, devem ser incluídos nas equações de fluxo em rede para manter a conservação de fluxo dos nós demandas originais e dos nós estoques originais.

Sejam $\hat{\mathbf{x}}^{\mathrm{k}}$ : vetor de fluxo do produto $\mathrm{k}$ através da rede $\hat{\mathfrak{g}} ; \hat{\mathbf{A}}$ : matriz incidência nó-arco de $\hat{g}$. Então,

$$
\hat{\mathbf{A}} \hat{\mathbf{x}}=\hat{\mathbf{b}}^{k} \text {, onde } \hat{\mathbf{b}}^{\mathbf{k}}=\left[\begin{array}{l}
0 \\
\vdots \\
\hat{b}_{s_{k}} \\
0 \\
\vdots \\
0 \\
\hat{b}_{t_{k}} \\
0 \\
\vdots \\
0
\end{array}\right] ; \text { e } \sum_{\mathbf{k}=1}^{K} \hat{\mathbf{D}}^{\mathbf{k}} \leq \mathbf{r}, \text { onde } \quad \hat{\mathbf{D}}^{\mathbf{k}}=\begin{array}{|l|l|}
\mathbf{D}^{\mathbf{k}} & \mathbf{0} \\
\hline
\end{array}
$$

Depois da transformação, o PPEA pode ser apresentado como:

$$
\begin{array}{ll}
\text { Minimizar } & \sum_{\mathrm{k}=1}^{\mathrm{K}} \mathbf{c}^{\mathrm{k}} \mathbf{x}^{\mathrm{k}} \\
\text { sujeito } \quad & \sum_{\mathrm{k}=1}^{\mathrm{K}} \mathbf{D}^{\mathrm{k}} \mathbf{x}^{\mathrm{k}} \leq \mathbf{r} \\
& \hat{\mathbf{A}} \hat{\mathbf{x}}=\hat{\mathbf{b}}^{\mathrm{k}} \mathrm{k}=1, \ldots, \mathrm{K} \\
& \hat{\mathbf{x}} \geq \mathbf{0} \\
& \overline{\mathbf{x}}_{\mathrm{s}_{\mathrm{k}}^{\mathrm{s}}}^{\mathrm{k}} \leq \mathrm{b}_{\mathrm{s}}^{\mathrm{k}} \text { para cada } \hat{\mathrm{s}}_{\mathrm{k}} \in \hat{S}, \mathrm{~s} \in S, \mathrm{k}=1, \ldots, \mathrm{K} \\
& \overline{\mathbf{x}}_{\mathrm{t}_{\mathrm{k}}}^{\mathrm{k}} \leq \mathrm{b}_{\mathrm{t}}^{\mathrm{k}} \text { para cada } \mathrm{t} \in T, \hat{\mathrm{t}}_{\mathrm{k}} \in T, \quad \mathrm{k}=1, \ldots, \mathrm{K}
\end{array}
$$


onde,

$$
\begin{aligned}
& \overline{\mathrm{x}}_{\hat{\mathrm{s}}_{\mathrm{k}}^{\mathrm{s}}}^{\mathrm{k}} \text { é o fluxo sobre o arco a partir do nó } \hat{\mathrm{s}}_{\mathrm{k}} \in \hat{S} \text { para nó } \mathrm{s} \in S \text { do produto } \mathrm{k} ; \\
& \overline{\mathrm{x}}_{\hat{t}_{\mathrm{k}}}^{\mathrm{k}} \text { é o fluxo do produto } \mathrm{k} \text { sobre a partir do nó } \mathrm{t} \in T \text { para o nó } \hat{\mathrm{t}}_{\mathrm{k}} \in T ; \\
& \overline{\mathbf{x}}_{\mathrm{s}_{\mathrm{k}}^{\mathrm{s}}}^{\mathrm{k}} \leq \mathrm{b}_{\mathrm{s}}^{\mathrm{k}} \text { e } \overline{\mathrm{x}}_{\hat{t}_{\mathrm{k}}}^{\mathrm{k}} \leq \mathrm{b}_{\mathrm{t}}^{\mathrm{k}} \text { são incluídas como desigualdades para garantir que na solução }
\end{aligned}
$$

factível ótima o fluxo sobre o arco $\overline{\mathrm{x}}_{\hat{\mathrm{k}}_{\mathrm{k}} \mathrm{s}}^{\mathrm{k}}$ e $\overline{\mathrm{x}}_{\hat{t}_{\mathrm{k}}}^{\mathrm{k}}$ sejam iguais a demanda e estoque do problema original PPE.

A matriz de restrições para o PPEA ;e dada por:

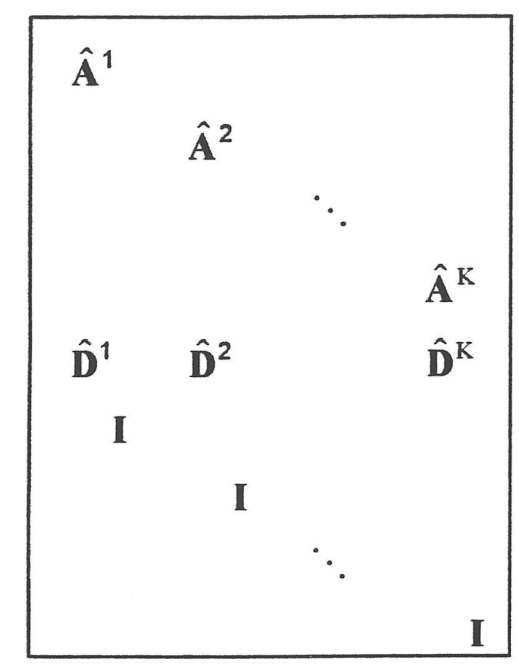

Figura 4.3 Matriz de restrições para o PPEA

No problema do PPEA, o vetor de estoques/demandas possui somente dois elementos não nulos, como no POD, entretanto as restrições de limite (capacidade) sobre os fluxos nos arcos $\overline{\mathrm{X}}_{\hat{S}_{\mathrm{k}} \mathrm{s}}^{\mathrm{k}}$ e $\overline{\mathrm{x}}_{\mathfrak{t}_{\mathrm{k}}}^{\mathrm{k}}$ torna esta formulação diferente da formulação do POD.

As restrições sobre o limite de $\overline{\mathrm{x}}_{\hat{\mathrm{s}}_{\mathrm{k}} \mathrm{s}}^{\mathrm{k}}$ e $\overline{\mathrm{x}}_{\hat{\mathrm{t}}_{\mathrm{k}}}^{\mathrm{k}}$, podem ser incluídas ou no problema mestre ou nos subproblemas. Se na decomposição estas restrições permanecerem no subconjunto de restrições, o problema mestre formado será igual ao problema mestre da formulação dada em (4.5), entretanto, nos subproblemas, para cada produto $\mathrm{k}$ existirá um limite inferior e superior sobre os arcos adicionais $\left(\hat{s}_{k}, s\right)$ e $\left(t, \hat{t}_{k}\right)$, de maneira que a solução deste subproblema será idêntica a solução dos subproblemas do PPE.

Por outro lado, se as restrições de limite sobre os $\operatorname{arcos}\left(\hat{\mathrm{s}}_{\mathrm{k}}, \mathrm{s}\right)$ e $\left(\mathrm{t}, \hat{\mathrm{t}}_{\mathrm{k}}\right)$ são considerados no problema mestre, as soluções do subproblemas serão semelhantes as soluções dos subproblemas obtidas pela formulação POD, ou seja, a solução dos subproblemas será um único caminho a partir de um nó super-estoque para um nó super-demanda. 
Observe que, como as restrições de capacidade dos arcos adicionais não permanecem nos subproblemas, as soluções caminhos mínimos obtidas pelos subproblemas são infactíveis para o PPE original. Pois a demanda do nó super-estoque dos subproblemas que devem ser resolvidos é dada por :

$$
\hat{b}_{\mathrm{s}_{\mathrm{k}}}^{\mathrm{k}}=\sum_{\mathrm{s} \in S} \mathrm{~b}_{\mathrm{s}}^{\mathrm{k}}=\sum_{\mathbf{t} \in T} \mathrm{~b}_{\mathrm{t}}^{\mathrm{k}}
$$

Logo, as soluções caminhos mínimos dos subproblemas devem necessariamente enviar um fluxo total $\hat{b}_{\mathrm{s}_{\mathrm{k}}}^{\mathrm{k}}$ a partir do nó super-estoque $\hat{\mathrm{s}}_{\mathrm{k}}$ para um único nó estoque original $\mathrm{s}$.

Esta solução caminho mínimo obtida pelos subproblemas é infactível para PPE, é então factibilizada no problema mestre, através das restrições de capacidade sobre os arcos adjacentes a todos os nós super-estoques e super-demandas.

Embora as restrições sobre os arcos adicionais estejam na forma de desigualdade no problema mestre restrito do PPEA, a decomposição faz com que sejam satisfeitas para como igualdade por causa das restrições de conservação de fluxo nos subproblemas.

Portanto, assim que uma solução inicial factível é obtida para o problema mestre restrito, esta solução satisfaz as restrições de estoque/demanda originais da formulação do PPE.

Para obter uma solução inicial factível para cada produto k, deve existir pelo menos um caminho passando por cada um dos nós estoques e demandas originais no problema mestre.

Como os subproblemas geram uma única solução caminho mínimo a partir de um nó superestoque para um nó super-demanda a cada iteração. Portanto, para obter uma solução inicial factível para um produto $\mathrm{k}$, devem ser gerados tantos caminhos mínimos quantos forem necessário para passar por todos os nós estoques e demandas originais.

Considere o exemplo dado a seguir, onde existem dois itens que devem percorrer a rede, com 3 nós estoques, 3 nós demandas e 2 nós intermediários

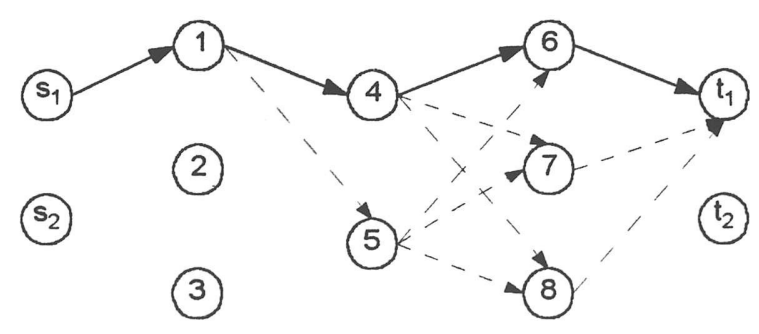

Figura 4.8:Solução do Subproblema do PPEA

A figura acima mostra uma solução, que será adicionada como uma coluna $\hat{s}_{1} \rightarrow \hat{t}_{1}$ no problema mestre restrito. Entretanto para satisfazer todas as restrições de estoque/demanda para o produto $\mathrm{k}=1$, o problema mestre também deve ter colunas associadas com os $\operatorname{arcos}\left(\hat{\mathrm{s}}_{1}, 2\right),\left(\hat{\mathrm{s}}_{1}, 3\right)$, $\left(7, \hat{\mathrm{t}}_{1}\right)$ e $\left(8, \hat{\mathrm{t}}_{1}\right)$. 
Para satisfazer estas restrições, o subproblema para o produto 1 deve ser resolvido pelos menos três vezes, gerando os caminhos:

$\hat{\mathrm{s}}_{1},\left(\hat{\mathrm{s}}_{1}, 1\right), 1,(1,4), 4,(4,5), 6\left(6, \hat{\mathrm{t}}_{1}\right)$;

$\hat{\mathrm{s}}_{1},\left(\hat{\mathrm{s}}_{1}, 2\right), 2,(2,4), 4,(4,7), 7\left(7, \hat{\mathrm{t}}_{1}\right)$;

$\hat{s}_{1},\left(\hat{s}_{1}, 3\right), 3,(3,5), 5,(5,8), 8\left(8 \hat{t}_{1}\right)$.

O subproblema deve escolher 3 dentre os 18 caminhos possíveis, entre o nó super-estoque $\hat{s}_{1}$ e o nó super-demanda $\hat{t}_{1}$, antes de obter uma solução inicial factível para o problema mestre restrito.

Os caminhos passando por todos os nós estoques e demandas originais para o produto 2 , também devem ser gerados, para que as restrições de conservação de fluxo sejam satisfeitas e uma solução factível para o problema mestre restrito seja obtida. 


\section{Comentários Finais}

$\mathrm{Na}$ primeira parte deste trabalho foram revisados propriedades e conceitos que possibilitaram um estudo sobre os modelos de fluxo em redes (com um único produto), tais como: o problema de fluxo em rede de custo mínimo, o do caminho mínimo e suas váriações, bem como a especializaçãodo método simplex.

Tal estudo preliminar se fez necessário para uma melhor compreensão das modelagens e métodos do problema de multifluxo, enfatizado neste trabalho.

Numa primeira formulação do problema de multifluxo chamada problema produtoespecífico, onde um produto é definido como um item específico, foram estudadas três abordagens de solução: o particionamento, a decomposição recurso-diretor e a decomposição de DantzigWolfe.

Para concluir nossos estudos, foram apresentadas outras duas definições para produto, implicando em outras duas formulações distintas para o problema de multifluxo: Problema destino-específico (PDE) baseada em árvores e o Problema origem-destino-específicos (POD) baseada em caminhos.

Utilizando a decomposição de Dantzig-Wolfe em ambas as formulações, pode-se observar e verificar, a despeito de implementações computacionais, que o número de subproblemas a serem resolvidos a cada iteração da decomposição de Dantzig-Wolfe, é muito maior para a formulação baseada em caminhos (POD) do que para a formulação baseada em árvores (PDE), e consequentemente, um número maior de restrições de convexidade no problema mestre do POD. Entretanto, o número de pontos extremos para os subproblemas na formulação POD é muito menor do que o número de pontos extremos dos subproblemas para a formulação PDE, resultando num desempenho computacional favoravel à primeira formulação, conforme relatado nos estudos de [Jones et al. - 93].

Para a formulação de produto-específico (PPE), o qual não é equivalente às outras formulações (PDE) e (POD), foi apresentado uma formulação equivalente baseada em caminhos 
(em oposição à formulação original baseada em árvores), aumentando o número de variáveis e restrições e as conclusões computacionais obtidas por [Jones et al. - 93] foram análogas a anterior.

Tais resultados contrariam a crença de que modelos mais reduzidos sejam mais fáceis de serem resolvidos.

A idéia de mudar a formulação do problema de multifluxo para tornar um algoritmo de otimização mais eficiente pode ser aplicado a outros métodos existentes tais como: o método de pontos interiores para otimização bloco angular ou a Decomposição Paralela de problemas de multifluxo em redes usando um algoritmo de Penalidade Linear Quadrátida, observado por [Jones et al. -93].

Outras formulações para o problema podem ainda ser exploradas. Por exemplo, a formulação arco-caminho (neste trabalho foi estudada apenas a formulação nó-arco). É interessante observar que o problema mestre da decomposição Dantzig-Wolfe para a formulação problema origem-destino específicos foi usado por [Tomlin-66] para mostrar a equivalência matemática entre estas formulações.

Em outro trabalho recente [Farvolden et al. 93] estudaram a especialização do particionamento primal usando a formulação arco-caminho para resolver o problema mestre resultante da decomposição Dantzig-Wolfe quando esta é aplicada para problemas de multifluxo em redes com uma estrutura produto origem-destino específicos.

O impacto das várias formulações poderiam ainda ser estudadas, nos diversos algoritmos já existentes quando são aplicados para resolver algum problema em especial, indicando que muita pesquisa pode ser realizada nesta área. 


\section{Apêndice A}

\section{Teoria dos Grafos}

\section{A.1 Resultados e definições da teoria dos grafos}

Grafo orientado: Um grafo orientado $q=[n, \mathscr{A}]$ consiste de um conjunto $n=\{1, \ldots, m\}$ de $m$ nós e um conjunto $\mathscr{A}=\left\{e_{1}, e_{2}, \ldots, e_{\mathrm{n}}\right\}$ de $n$ arcos, onde um arco $e_{\mathrm{j}}$ é representado pelo par $e_{\mathrm{j}}=(\mathrm{F}(\mathrm{j}), \mathrm{T}(\mathrm{j}))$. Diz-se que o arco $e_{\mathrm{j}}$ sai do nó $\mathrm{F}(\mathrm{j})$ e aponta para o nó $\mathrm{T}(\mathrm{j})$. Neste trabalho consideraremos somente arcos para os quais $F(j) \neq T(j)$.

Rede orientada: Uma rede orientada é um grafo orientado cujos nós e arcos tem um valor numérico associado (custos, capacidades, estoques e demandas).

No desenvolvimento desse trabalho não será feita distinção entre os termos grafo e rede. Ambos serão usados com o mesmo sentido. Será considerado somente redes com um número fínito $m$ de nós e um número finito $n$ de arcos.

Assumiremos também que não existem arcos múltiplos na rede, isto é, não existem arcos $e_{\mathrm{j}}=$ $e_{\mathrm{k}}$, tais que $\mathrm{F}(\mathrm{j})=\mathrm{F}(\mathrm{k})$ e $\mathrm{T}(\mathrm{j})=\mathrm{T}(\mathrm{k})$. Se existir tais arcos, um deles pode ser substituído por dois outros arcos. Por exemplo, $(\mathrm{F}(\mathrm{j}), m+1)$ e $(m+1, \mathrm{~T}(\mathrm{j}))$, onde $m+1$ é um novo nó.

As figuras a seguir exibe a representação geométrica de um grafo com arcos múltiplos e sem arcos múltiplos.

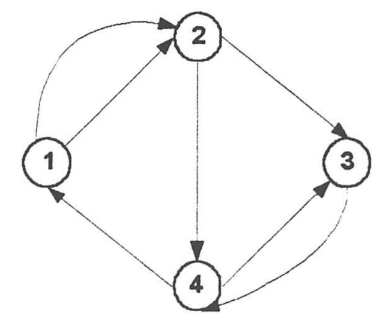

Figura A1: Grafo orientado com arcos múltiplos

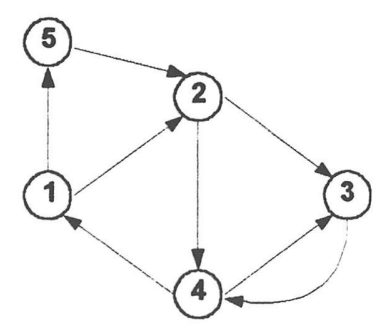

Figura A2 : Grafo orientado sem arcos múltiplos

Grafo próprio: Diz-se que um grafo $q=[\eta, \mathscr{A}]$ é um grafo próprio se número de nós $m \geq 2$ e o número de $\operatorname{arcos} n \geq 1$. 


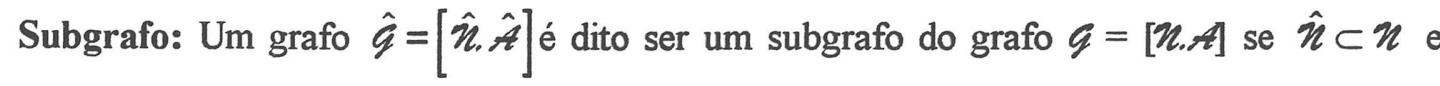
$\hat{A} \subset \mathscr{A}$.

Subgrafo gerador: Um grafo $\hat{q}=[\hat{\eta}, \hat{\hbar}]$ é dito ser um subgrafo gerador para o grafo $\mathscr{G}=[\boldsymbol{n}, \mathscr{A}]$ se $\hat{x}=\boldsymbol{x}$ e $\hat{t} \subseteq \mathcal{A}$.

Caminho: Uma sequência $\mathrm{P}=\left\{s_{1}, e_{\mathrm{j}_{1}}, s_{2}, e_{\mathrm{j}_{2}}, \ldots, s_{\mathrm{n}}, e_{\mathrm{j}_{\mathrm{n}}}, s_{\mathrm{n}+1}\right\}$ tendo pelo menos um arco é chamada de caminho em um grafo $\mathcal{G}$ se seus elementos ímpares são nós distintos de $\mathcal{G}$ e seus elementos pares são arcos de $\mathcal{q}$, onde cada arco $e_{\mathrm{j}_{\mathrm{i}}} \in\left\{\left(s_{\mathrm{i}}, s_{\mathrm{i}+1}\right),\left(s_{\mathrm{i}+1}, s_{\mathrm{i}}\right)\right\}$.

Proposição A.1: Os arcos de um caminho são distintos.

Pode-se classificar os arcos de um caminho em dois grupos: arcos forward e arcos backward. Um arco é forward se $e_{\mathrm{j}_{\mathrm{i}}}=\left(s_{\mathrm{i}}, s_{\mathrm{i}+1}\right)$ e é um arco backward se $e_{\mathrm{j}_{\mathrm{i}}}=\left(s_{\mathrm{i}+1}, s_{\mathrm{i}}\right)$..

Na figura $A 2$, considere o caminho dado por $P=\{1,(1,2), 2,(2,3), 3,(4,3), 4\}$. Os arcos $(1,2)$ e $(2,3)$ são arcos forward e $(4,3)$ é um arco backward.

Caminho orientado: Um caminho orientado é um caminho sem arcos backward.

Ciclo: Uma sequência finita $\mathrm{C}=\left\{s_{1}, e_{\mathrm{j}_{1}}, s_{2}, e_{\mathrm{j}_{2}}, \ldots, s_{\mathrm{n}}, e_{\mathrm{j}_{\mathrm{n}}}, s_{\mathrm{n}+1}\right\}$ tendo pelo menos dois arcos é chamado ciclo em $\mathcal{G}$ se a subsequência $\left\{s_{1}, e_{\mathrm{j}_{1}}, s_{2}, e_{\mathrm{j}_{2}}, \ldots, e_{\mathrm{j}_{\mathrm{n}-1}}, s_{\mathrm{n}}\right\}$ é um caminho em $\mathcal{q}$, onde $s_{1}=s_{\mathrm{n}+1}$ e $e_{\mathrm{j}_{\mathrm{n}}} \neq e_{\mathrm{j}_{1}}$.

Proposição A.2: Os arcos de um ciclo são distintos.

Ciclo orientado: Um ciclo orientado $\mathrm{C}=\left\{s_{1}, e_{\mathrm{j}_{1}}, s_{2}, e_{\mathrm{j}_{2}}, \ldots, s_{\mathrm{n}}, e_{\mathrm{j}_{\mathrm{n}}}, s_{\mathrm{n}+1}\right\}$ é um caminho orientado juntamente com o $\operatorname{arco}\left(s_{\mathrm{n}}, s_{1}\right)$.

Conectividade: Dois nós $i$ e $t$ são conexos se o grafo contém pelo menos um caminho a partir do nó $i$ para o nó $t$. Um grafo é conexo se todo par de nós distintos é conexo.

Árvore: Uma árvore é um grafo conexo que não contém ciclos.

Árvore Geradora: Diz-se que uma árvore é geradora para o grafo $q$, se esta árvore for um subgrafo gerador para $\mathfrak{q}$.

Proposição A.3: Todo grafo conexo $q=[\eta, \mathscr{A}]$ com $n \neq \varnothing$ tem um subgrafo que é uma árvore geradora.

As figuras a seguir ilustra alguns exemplos baseado no grafo da figura A2.

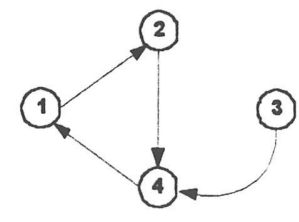

Figura A3.1: Subgrafo com um ciclo orientado

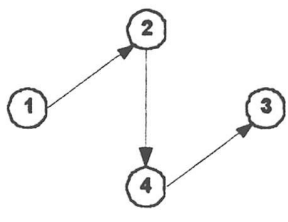

Figura A3.2:. Subgrafo com um caminho orientado do nó 1 para o nó 3 


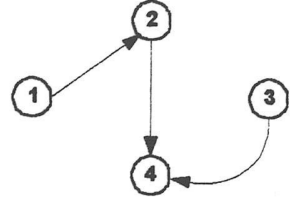

Figura A3.3:. Árvore

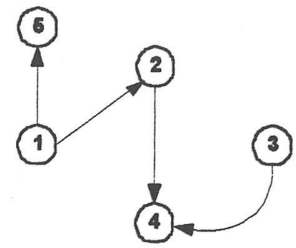

Figura A3.4:. Árvore geradora

Proposição A.4: Para o grafo $7=[\varkappa, \not]$ tendo $m \geq 1$ nós e $n$ arcos, as seguintes afirmações são equivalentes:

(1) 7 é uma ávore;

(2) Para cada par de nós distintos $(p, q)$ de 7. existe um único caminho que liga o nó p com o nó q;

(3) 7 tem $n=m-1$ arcos e é conexa;

(4) 7 tem $n=m-1$ arcos e não tem ciclos.

\section{A.2 Representações para redes}

Para representar uma rede, necessita-se armazenar dois tipos de informação: (1) a topologia da rede, isto é, a estrutura de arcos e nós da rede; (2) os dados, tais como custos, capacidades, estoques e demandas associados com os arcos e nós da rede.

A.2.1 Matriz adjacência nó-nó: A estrutura de uma rede pode ser representada por uma matriz $m \times m$ chamada matriz adjacência nó-nó, onde cada linha e coluna corresponde a um nó na rede, definida por:

$$
\mathrm{A}_{\mathrm{ij}}=\left\{\begin{array}{l}
1 \text { se }(\mathrm{i}, \mathrm{j}) \in \mathscr{A} \\
0 \text { caso contrario }
\end{array}\right.
$$

A matriz adjacência nó-nó tem $m^{2}$ elementos, dos quais somente $n$ (número de arcos) são diferentes de zero.

Exemplo 1: A matriz adjacência nó-nó correspondente ao grafo dado na fig A2 é dada por: nós

$$
\mathbf{A}=\left[\begin{array}{llllll}
1 & 2 & 3 & 4 & 5 & \text { nós } \\
0 & 1 & 0 & 0 & 1 \\
0 & 0 & 1 & 1 & 0 \\
0 & 0 & 0 & 1 & 0 \\
1 & 0 & 1 & 0 & 0 \\
0 & 1 & 0 & 0 & 0
\end{array}\right] \begin{aligned}
& 1 \\
& 2 \\
& 3 \\
& 4 \\
& 5
\end{aligned}
$$

A.2.2 Matriz incidência nó-arco: A estrutura de uma rede também pode ser representada por uma matriz $m \times n$, que contém uma linha para cada nó da rede e uma coluna correspondente a cada arco , definida como:

$$
\mathrm{A}_{\mathrm{ij}}=\left\{\begin{array}{l}
+1 \text { se } o \text { arco } j \text { sai do no } i \\
-1 \text { se } 0 \text { arco } j \text { aponta para o no } i \\
0 \text { caso contrario }
\end{array}\right.
$$


Uma característica desta matriz é que cada coluna correspondente ao arco $e_{\mathrm{j}}$ tem exatamente dois elementos não nulos, com +1 na linha correspondente ao nó $F(j)$ e -1 na linha correspondente ao nó $\mathrm{T}(\mathrm{j})$. Uma coluna da matriz incidência nó-arco também pode ser representada por $\mathrm{e}^{\mathrm{F}(\mathrm{j})}-\mathrm{e}^{\mathrm{T}(\mathrm{j})}$, onde $\mathrm{e}^{\mathrm{F}(\mathrm{j})}$ é um vetor com $m$ elementos, com "1" na $\mathrm{F}(\mathrm{j})$-ésima componente e zero nas demais.

Exemplo 2: A matriz incidência nó-arco correspondente a figura A2 é dada por:

$$
\mathbf{A}=\left[\begin{array}{cccccccc}
1 & 1 & 0 & 0 & 0 & 0 & -1 & 0 \\
0 & -1 & 1 & 1 & 0 & 0 & 0 & -1 \\
0 & 0 & 0 & -1 & 1 & -1 & 0 & 0 \\
0 & 0 & -1 & 0 & -1 & 1 & 1 & 0 \\
-1 & 0 & 0 & 0 & 0 & 0 & 0 & 1
\end{array}\right] \begin{gathered}
1 \\
2 \\
3 \\
4
\end{gathered}
$$

A matriz incidência nó-arco será a representação usada neste trabalho. Esta representação é importante, pois apresenta as restrições de conservação de fluxo apropriadamente.

Sequência de orientação: Para qualquer caminho (ou ciclo) P com $n$ arcos, define-se a sequência de orientação $\mathrm{O}(\mathrm{P})$ tendo $n$ elementos como sendo:

$$
\mathrm{O}_{\mathrm{j}_{\mathrm{i}}}(\mathrm{P})= \begin{cases}+1 & \text { se } e_{\mathrm{j}_{\mathrm{i}}}=\left(s_{\mathrm{i}}, s_{\mathrm{i}+1}\right) \\ -1 & \text { se } e_{\mathrm{j}_{\mathrm{i}}}=\left(s_{\mathrm{i}+1}, s_{\mathrm{i}}\right)\end{cases}
$$

Proposição A.5: Seja a sequência $\mathrm{P}=\left\{s_{1}, e_{\mathrm{j}_{1}}, s_{2}, e_{\mathrm{j}_{2}}, \ldots, s_{\mathrm{n}}, e_{\mathrm{j}_{\mathrm{n}}}, s_{\mathrm{n}+1}\right\}$ um caminho em um grafo próprio $q$ com $\mathbf{A}$ a matriz incidência nó-arco. Então:

$$
\sum_{\mathrm{i}=1}^{\mathrm{n}} \mathrm{O}_{\mathrm{j}_{\mathrm{i}}}(\mathrm{P}) \mathbf{A}^{\mathbf{j}_{\mathrm{i}}}=\mathbf{e}^{s_{1}}-\mathbf{e}^{\mathrm{s}_{\mathrm{n}+1}}
$$

Proposição A.6: Seja $\mathrm{C}=\left\{s_{1}, e_{\mathrm{j}_{1}}, s_{2}, e_{\mathrm{j}_{2}}, \ldots, s_{\mathrm{n}}, e_{\mathrm{j}_{\mathrm{n}}}, s_{\mathrm{n}+1}\right\}$ um ciclo em um grafo próprio $q$ com $\mathbf{A}$ a matriz incidência nó-arco. Então $\sum_{\mathrm{i}=1}^{\mathrm{n}} \mathbf{O}_{\mathrm{j}_{\mathrm{i}}}(C) \mathbf{A}^{\mathrm{j}_{\mathrm{i}}}=0$.

Corolário A.7: Considere o ciclo na proposição A.6. Então $\left\{\mathbf{A}^{\mathrm{j}_{\mathrm{i}}}: i=1, \ldots, n\right\}$ é linearmente dependente.

Proposição A.8: Seja A a matriz incidência nó-arco para um grafo próprio $\mathcal{G}$. Seja $7=[\varkappa . \mathscr{A}]$ um subgrafo de $\mathcal{q}$ que é uma árvore tendo pelo menos dois nós. Então $\left\{\mathbf{A}^{\mathrm{j}_{\mathrm{i}}}: \boldsymbol{e}_{\mathrm{j}_{\mathrm{i}}} \in \mathscr{A}\right\}$ é linearmente independente.

Proposição A.9: Seja A a matriz incidência nó-arco para um grafo próprio q que é conexo e tem $m$ nós. Então o posto(A) $=m-1$.

Proposição A.10: Seja A a matriz incidência nó-arco para um grafo próprio $\mathscr{q}=[\varkappa \cdot \not{A}]$, onde $q$ tem $m$ nós. Seja $\hat{\mathscr{A}}$ um subconjunto de $\mathscr{A}$ tal que $\left\{\mathrm{A}^{\mathrm{j}_{\mathrm{i}}}: e_{\mathrm{j}_{\mathrm{i}}} \in \hat{\mathscr{A}}\right\}$ é linearmente independente e $\hat{A}$ tem $m-1$ arcos. Então $7=[\varkappa . \hat{\mathscr{t}}]$ é uma árvore. 


\section{A.3 Transformações em redes}

Algumas vezes necessita-se transformar uma rede dada para fazer algumas simplicações ou apresentar a rede em uma forma padrão.

Nesta seção descreveremos algumas destas transformações, que se aplicam para o problema de fluxo em rede de custo mínimo e seua casos especiais.

\section{A .3.1 Remover a capacidade dos arcos}

Se um arco $e_{\mathrm{j}}$ tem capacidade positiva $u_{\mathrm{j}}$, pode-se remover essa capacidade, tornando-o um arco não-capacitado fazendo a seguinte transformação na rede.

Adicionando a variável de folga $s_{j} \geq 0$ na restrição de capacidade $x_{j} \leq u_{j}$, pode-se escrevê-la na forma de igualdade, $x_{j}+s_{j}=u_{j}$. Multiplicando ambos os lados por $(-1)$ obtem-se:

$$
-x_{j}-s_{j}=-u_{j} .
$$

A equação acima é tratada agora como uma restrição de conservação de fluxo de um nó adicional $(n+1)$.

Note que, a variável $x_{j}$ aparece em três restrições de conservação de fluxo e $s_{j}$ em somente uma. Para garantir que $x_{j}$ e $s_{j}$ apareçam em exatamente duas restrições de conservação de fluxo, subtrai-se a equação $-x_{j}-s_{j}=-u_{j}$ da restrição de conservação de fluxo do nó $T(j)$ (que contém a variável $\mathrm{x}_{\mathrm{j}}$ com sinal negativo).

Fazer essa transformação na rede é equivalente a adicionar um novo nó na rede, ou seja, adicionar uma linha na matriz incidência nó-arco.

Se todo arco na rede é capacitado, aplicando esta transformação na rede obtem-se uma rede não-capacitada bipartida.

\section{A .3.2 Inversão de arcos}

A inversão de arcos é usada para remover arcos com custo negativo $c_{j}<0$. Nesta transformação substitui-se a variável $\mathrm{x}_{\mathrm{j}}$ por $\mathrm{x}_{\mathrm{j}}=\mathrm{u}_{\mathrm{j}}-\mathrm{x}_{\mathrm{j}}{ }^{\prime}$, onde $\mathrm{u}_{\mathrm{j}}$ é a cpacidade sobre o $\operatorname{arco} e_{\mathbf{j}}$.

Fazendo essa substituição nas restrições de conservação de fluxo, diminui-se $b_{F(i)}$ por $u_{j}$ unidades e aumenta-se $b_{T(j)}$ por $u_{j}$ unidades e o $\operatorname{arco}(F(j), T(j))$ é substiuído pelo arco $(T(j), F(j))$. A nova variável $\mathrm{x}_{\mathrm{j}}{ }^{\prime}$ determina a quantidade de fluxo que movemos da capacidade total do fluxo sobre o arco $e_{j}$. Sustituindo na função objeiivo tem-se: $c_{j}\left(u_{j}-x_{j}{ }^{\prime}\right)=c_{j} u_{j}-c_{j} x_{j}{ }^{\prime}$,onde o custo negativo associado a uma variável de decisão foi removido.Essa transformação entretanto, requer que todos os arcos tenham capacidade finita. Quando esse não é o caso, assume-se que a rede não contém ciclos orientados com custo negativo, pois se a rede contém tais ciclos, o valor ótimo para o problema de fluxo em rede de custo mínimo é ilimitado. 


\section{Bibliografia}

[AHUJA - 93] Ahuja, R. K., Magnanti, T. L., Orlin, J. B., “Network Flows”, Prentice Hall, 1993.

[ASSAD - 78] Assad, A . A ., "Multicommodity Network Flows: A Survey", Networks, 8, 1, 37-92 (1978)

[BAZARAA/JARVIS - 77] Bazaraa, M. S., Jarvis, J. J., "Programming and Network lows" John Wiley \& Sons, (1977)

[DANTZIG/WOLFE - 61] Dantzig, G. B., Wolfe, P., "The Decomposition Algorithm for Linear Programs", Econometria, - Vol. 29 - nº 4, pp. 767 - 778, (1961)

[FARVOLDEN et al - 93] Farvolden, M. J., Powell, W. B., Lustig, I. J., "A Primal Partitioning Solution for Arc-Chain Formulation of a Multicommodity Network Flow Problem", Operations Research, 41,4,669-693 (1993)

[FLORENTINO - 90] Florentino, H.O., "Relaxação Lagrangeana em Programação Inteira", Dissertação de Mestrado, ICMSC - USP, (1990)

[GEOFFRION - 70] Geoffrion, A . M., "Primal Resource-Directive Approaches for Optimizing Nonlinear Decomposable Systems", Operations Research, 18,3,375-403 (1970)

[HARTMAN/LASDON - 72] Hartman, J. K., and Lasdon, L. S., "A Generalized Upper Bounding Algorithm for Multicommodity Network Flow Problems”, Networks, 1,333-354 (1972)

[HELD et al - 74] Held, M., Wolfe, P., Crowder, H., "Validation of Subgradient Optimization", Mathematical Programing, 6,62-88 (1974)

[HELGASON et al - 79] Helgason, R., Kenningotn, J., Lall, H., "A Polynomially Bounded Algorithm for a Singly Constained Quadratic Progam", Mathematical Programming, 338-343 (1979)

[JONES et al - 93] Jones, K.L., Lusting , I.J., Farvolden, J.M. ,Powell, W.,B., "Multicommodity Newtwork Flows: The Impact of Formulation on Decomposition", Mathematical Programming, Vol. 62, pp. 95 - 117, (1993)

[KENNINGTON - 77] Kenningotn, J., A “Survey of Linear Cost Multicommodity Network Flow" Operations Research,- Vol. 26 - nº 2, pp. 209 - 236, (1977)

[KENNINGTON/HELGASON - 80] Kennington, J., L., Helgason, R., V., “Algorithms for Network Programming", John Wiley \& Sons, (1980) 
[KENNINGTON/SHALABY - 77] Kennigton, J., Shalaby, M., “An Efective Subgradient Procedure for Minimal Cost Multicommodity Flow Problems", Managent Science - Vol. 23 - n 9, pp. 994 1004, (1977)

[LASDON - 72] Lasdon, L. S., "Optimization Theory for Large Systems", Macmillian Co., London, England, (1972)

[LUENBERGER - 84] Luenberger, D., G., "Linear and Nonlinear Programming", AddisonWesley Publishing Company, (1984)

[TOMLIN-66] Tomlin, J. A ,, "Minimum-Cost Multicommodity Network Flows", Operations Research, 14,45-51 (1966) 